\title{
Overview of Shelling for 2-Manifold Surface Reconstruction based on 3D Delaunay Triangulation
}

\author{
Maxime Lhuillier
}

Received: date / Accepted: date

The reference of this paper is: Maxime Lhuillier, Overview of Shelling for 2-Manifold Surface Reconstruction based on 3D Delaunay Triangulation, Journal of Mathematical Imaging and Vision, 59(2):318-340, 2017

This is the accepted manuscript version that is available at the webpage of the author. The final publication is available at Springer via

http://dx.doi.org/10.1007/s10851-017-0734-4
Maxime Lhuillier

Institut Pascal, UMR 6602 CNRS/UCA/SIGMA, 63178

Aubière cedex, France

Tel.: $+33(0) 473407593$

Fax: $+33(0) 473407262$

E-mail: maxime.lhuillier@univ-bpclermont.fr

\begin{abstract}
Recently, methods have been proposed to reconstruct a 2-manifold surface from a sparse cloud of points estimated from an image sequence. Once a 3D Delaunay triangulation is computed from the points, the surface is searched by growing a set of tetrahedra whose boundary is maintained 2-manifold. Shelling is a step that adds one tetrahedron at once to the growing set. This paper surveys properties that helps to understand the shelling performances: shelling provides most tetrahedra enclosed by the final surface but it can "get stuck" or block in unexpected cases.
\end{abstract}

Keywords Reconstruction - Volumetric models . Shellability · 3D Delaunay Triangulation · Star-Shapes

\section{Introduction}

In the recent years, a family of 2-manifold surface reconstruction methods have been proposed to deal with a sparse cloud of input points and their visibility information estimated from an image sequence. There are both batch [13] and incremental [23,15,16,18] variations. First a 3D Delaunay triangulation is computed from the sparse point cloud to divide up the space by a set $T$ of tetrahedra. Then the visibility information is used to associate a free-space score to every tetrahedron $\Delta \in T$, e.g. by counting the rays (line segments between points and camera poses used to reconstruct these points) that intersect $\Delta$. Last these scores are used for growing a set $O$ of tetrahedra in $T$ such that the boundary $\partial O$ of $O$ is maintained 2-manifold.

Several operations are designed for the $O$ growing. One of them adds one tetrahedron at once to $O$ and is closely related to the "shelling" process that is studied in Combinatorial Topology: Algorithm 1 in [13] generates a shelling in the $3 \mathrm{D}$ case if $O$ is initialized by a 
single tetrahedron. This greedy algorithm tries to add to $O$ the tetrahedron in $T \backslash O$ that has the largest visibility score (and that has a face triangle in $\partial O$ ) if $\partial O$ is still a 2-manifold. The goal of the paper is to survey the shelling properties in our surface reconstruction context, where $O$ is included in a 3D Delaunay triangulation and the $O$ initialization is not restricted to a single tetrahedron.

First Sec. 2 introduces prerequisites. Second, Sec. 3 provides our shelling definition and shows that it does not change the topology (number of connected components and genus) of $\partial O$ in almost all cases.

Third, Sec. 4 overviews shelling properties known in Combinatorial Topology, i.e. if $O$ is initialized by a single tetrahedron. At first glance, one could guess that a shelling started by a single tetrahedron in $T$ can reach/have every end value $O_{e} \subseteq T$ such that tetrahedron union $\cup O_{e}$ is homeomorphic to one tetrahedron. Surprisingly, this is wrong: some of these $O_{e}$ do not have such a shelling. This implies that no greedy algorithm [13,18] (whatever the free-space scoring of the tetrahedra) can reach $O_{e}$. Furthermore, even if there is a shelling that reaches $O_{e}$, a greedy algorithm can "get stuck" or block: it can generate another shelling with other tetrahedron choice(s) at the beginning such that the end value is strictly included in $O_{e}$.

Fourth, Sec. 5 studies shelling in the favorable case of star-shapes in a 3D Delaunay triangulation. We remind that $\cup O$ is star-shaped with respect to the center $\mathbf{c} \in \mathbb{R}^{3}$, if for every point $\mathbf{x} \in \cup O$, the line segment $\mathbf{x c} \subseteq \cup O$. More precisely, we show that there is a shelling that starts from $O_{s}$ and ends to $O_{e} \subseteq T$ if both $\cup O_{s}$ and $\cup O_{e}$ are star-shaped with respect to the same center. This generalizes a known case where $\cup O_{e}$ is convex (indeed, a convex set is star-shaped with respect to every of its point) and $O_{s}$ has a single tetrahedron. Then Sec. 5 explains why shelling provides most of the tetrahedra enclosed by the final surface in our context (shelling does not have excessive blocking).

Last, Sec. 6 provides examples of shelling blocking: a shelling starts from $O_{s}$ but cannot end to $O_{e}$ although $\cup O_{s}$ and $\cup O_{e}$ have the same topology. This should convince the reader that such cases really exist, although they don't in the $2 \mathrm{D}$ case [4] (i.e. by replacing tetrahedra by triangles). The examples are chosen to be as simple as possible. Sec. 6 introduces a family of sets $O$ that cannot be reached by a shelling started from a single tetrahedron (as in Sec. 4), which generalizes an example in [24]. It also introduces examples of visual artifact similar to that in Fig. 1 of [16] which cannot be removed by shelling alone (against the intuition).

\section{Main prerequisites}

The majority of prerequisites are in $[5,21,9,11,14]$.

\subsection{Simplicial complexes in $\mathbb{R}^{n}$}

Let integers $k \geq 0$ and $n>0$. A simplex $\sigma$ is the convex hull of $k+1$ points $\mathbf{v}_{0}, \cdots, \mathbf{v}_{k}$ in general position in $\mathbb{R}^{n} ; \sigma$ is a vertex if $k=0$, an edge if $k=1$, a triangle if $k=2$, a tetrahedron if $k=3$. The dimension of $\sigma$ is $k$. A simplex $\sigma^{\prime}$ is a face of $\sigma$ if $\sigma^{\prime}$ is the convex hull of some of the $\mathbf{v}_{i}$ above (we have $\sigma^{\prime} \subseteq \sigma$ ). A simplicial complex $K$ in $\mathbb{R}^{n}$ is a finite set of simplices in $\mathbb{R}^{n}$ such that (1) $\sigma^{\prime} \in K$ if $\sigma^{\prime}$ is a face of $\sigma \in K$ and (2) $\sigma \cap \sigma^{\prime}$ is empty or a face of $\sigma$ and $\sigma^{\prime}$ if both $\sigma$ and $\sigma^{\prime}$ are in $K$.

A $3 D$ Delaunay triangulation is a simplicial complex $K$ in $\mathbb{R}^{3}$ that meets several conditions. There is a set $T$ of tetrahedra such that the faces of the tetrahedra are the simplices in $K$. Let $V$ be the vertex set of $K$. The circumscribing sphere of every tetrahedron in $T$ does not contain a vertex in $V$ in its interior. The convex hull of $V$ is the union of the tetrahedra in $T$.

\subsection{Closure, pure simplicial complex, boundary}

Let $A$ be included in a simplicial complex. The closure $c(A)$ of $A$ is the set of all faces (including the vertices) of the simplices in $A$. If both $A$ and $B$ are included in a same simplicial complex, $c(A \cup B)=c(A) \cup c(B)$, $c(A \cap B) \subseteq c(A) \cap c(B)$. If $A \subseteq B, c(A) \subseteq c(B)$. We use notation $c\left(\sigma_{1}, \cdots \sigma_{k}\right)=c\left(\left\{\sigma_{1}, \cdots \sigma_{k}\right\}\right)$ for simplices $\sigma_{i}$.

Let $X$ be a simplicial complex. We say that $X$ is $k D$ pure if there are $\operatorname{simplices}(\mathrm{s}) \sigma_{i} \in X$ with a same dimension $k$ such that $X=c\left(\sigma_{1}, \cdots \sigma_{m}\right)$. A 3D Delaunay triangulation $K$ is $3 \mathrm{D}$ pure and meets $K=c(T)$ where $T$ is the set of all tetrahedra in $K$.

If $A$ is a set of simplices with a same dimension $k>0$, the boundary $\partial A$ of $A$ is the set of all simplices of dimension $k-1$ in $c(A)$ that are faces of exactly one simplex in $A$. The boundary $\partial \sigma$ of a simplex $\sigma$ is $\partial\{\sigma\}$.

\subsection{Abstract simplicial complexes}

Let $\mathcal{V}$ be a finite set. An abstract simplicial complex $\mathcal{S}$ is a set of subsets of $\mathcal{V}$ such that $A \in \mathcal{S}$ and $B \subset A$ imply $B \in \mathcal{S}$. It is implicitly defined by every simplicial complex $K$ as follows: $\mathcal{V}$ is the vertex set of $K$ and $\mathcal{S}$ is the set of the vertex sets of the $K$ simplices. Conversely, $K$ is a realization of $\mathcal{S}$ in $\mathbb{R}^{n}$. The elements of $\mathcal{S}$ are called (abstract) simplices; their faces are their subsets. 
The definitions of edge/triangle/tetrahedron, closure, $k \mathrm{D}$ pure and boundary still hold for abstract simplices and abstract simplicial complexes, replacing simplices by abstract simplices and using their same inclusion relations. Same notations are used for a simplicial complex and its abstract version, and also for a simplex and its abstract version. We use bold fonts for vertices, e.g. $\mathbf{a b}$ is an edge (abstract or non-abstract).

Let $c(T)$ be a 3D Delaunay triangulation using a non-empty set $T$ of tetrahedra. Every triangle in $c(T)$ is included in exactly two tetrahedra in $c(T)$, except those in $\partial T$ (we have $\partial T \neq \emptyset$ ). These exceptions are removed as in $[2,14]$ to make easier proofs. Let $\mathbf{v}_{\infty}$ be an abstract vertex that is different to those in $c(T)$. For every triangle $\mathbf{a b c} \in \partial T$, we create a new tetrahedron $\mathbf{a b c v} \mathbf{v}_{\infty}$ by adding $\mathbf{v}_{\infty}$ to the set abc. Let $T^{\infty}=$ $T \cup\left\{\mathbf{a b c v}_{\infty}, \mathbf{a b c} \in \partial T\right\}$. Now $T \subset T^{\infty}$ and every triangle in $c\left(T^{\infty}\right)$ is a face of exactly 2 tetrahedra in $T^{\infty}$. Notations $T$ and $T^{\infty}$ are used in the whole paper.

\subsection{2-manifolds (with boundary), $k$-spheres, $k$-balls}

Let $A \subseteq \mathbb{R}^{n}$ and $B \subseteq \mathbb{R}^{m}$. If there is a bijective and continuous function between $A$ and $B$ such that the inverse function is also continuous, $A$ and $B$ are homeomorphic. We say that $A$ is a 2-manifold with boundary if every point $\mathbf{x} \in A$ has a neighborhood in $A$ that is homeomorphic to $\mathbb{R}^{2}$ or $\mathbb{R} \times \mathbb{R}^{+}\left(\mathbb{R}^{+}\right.$includes 0$) ; A$ is a 2-manifold if every point $\mathbf{x} \in A$ has a neighborhood in $A$ that is homeomorphic to $\mathbb{R}^{2} ; A$ is a $k$-ball $(k \in\{1,2,3\})$ if it is homeomorphic to $\left\{\mathbf{x} \in \mathbb{R}^{k},\|\mathbf{x}\| \leq 1\right\} ; A$ is a $k$-sphere $(k \in\{1,2\})$ if it is homeomorphic to $\left\{\mathbf{x} \in \mathbb{R}^{k+1},\|\mathbf{x}\|=\right.$ 1\}. 2-spheres are 2-manifolds, 2-manifolds and 2-balls are 2-manifolds with boundary.

Let $Y \subseteq c(T)$. We define $|Y|=\cup Y=\cup_{\sigma \in Y} \sigma$ (here $\sigma$ is a convex hull, it is not an abstract simplex). We say that $Y$ is homeomorphic to $B$ if $|Y|$ is homeomorphic to $B$. Thus $Y$ can be a 2-manifold/2-manifold with boundary/k-sphere/ $k$-ball. Here are examples: a triangle in $c(T)$ is a 2 -ball, $\partial T$ is a 2 -sphere, $\partial \Delta$ is a 2 -sphere and $\Delta$ is a 3 -ball if tetrahedron $\Delta \in c(T)$.

Let vertex series $\mathbf{v}_{1} \cdots \mathbf{v}_{m}$ where $m \geq 2$. We use notation $\mathbf{v}_{1}-\mathbf{v}_{2^{-}} \cdots-\mathbf{v}_{m}$ for the set of edges $\mathbf{v}_{1} \mathbf{v}_{2}, \mathbf{v}_{2} \mathbf{v}_{3}, \cdots$, $\mathbf{v}_{m-1} \mathbf{v}_{m}$. If the $\mathbf{v}_{i}$ are distinct and every $\mathbf{v}_{i} \mathbf{v}_{i+1} \in$ $c(T), \mathbf{v}_{1}-\mathbf{v}_{2}-\cdots-\mathbf{v}_{m}$ is a 1-ball. A cycle is an edge set $\mathbf{v}_{1}-\cdots-\mathbf{v}_{m}-\mathbf{v}_{1}$ such that $m \geq 3$ and the $\mathbf{v}_{i}$ are distinct. A cycle is a 1-sphere if all its edges are in $c(T)$.

Here we merge Theorem 4 and Lemma 4 in [14]:

Theorem 1 Let $O \subset T^{\infty}$ be such that $\partial O \subset c(T)$ and $\partial O$ is a 2-manifold. If $\Delta$ is a tetrahedron in $T \backslash O$, $\partial(O \cup\{\Delta\})$ is a 2-manifold iff $c(O) \cap c(\Delta)$ is $2 D$ pure.

\subsection{2-manifold criterion for simplicial complexes}

If $\mathbf{v}$ is a vertex and $E$ is a set of edges, $\mathbf{v} \times E$ is the set of the triangles defined by joining $\mathbf{v}$ and every edge in $E: \mathbf{v} \times E=\{\mathbf{v a b}, \mathbf{a b} \in E\}$. Let $X$ be a set of triangles in $c(T)$. If a vertex $\mathbf{v} \in c(X)$, we define $X_{\mathbf{v}}$ by the set of all triangles in $X$ that include $\mathbf{v}$. There is a set $E_{\mathbf{v}}$ of edges such that $X_{\mathbf{v}}=\mathbf{v} \times E_{\mathbf{v}}$. If $E_{\mathbf{v}}$ is not a cycle, $\mathbf{v}$ is a singular vertex of $X$.

According to $[21,1,14], X$ is a 2-manifold iff for every vertex $\mathbf{v} \in c(X)$, there is a cycle $\mathbf{a}_{*}$ such that $X_{\mathbf{v}}=\mathbf{v} \times \mathbf{a}_{*}$. Thus there are distinct vertices $\mathbf{a}_{i} \in c\left(X_{\mathbf{v}}\right)$ such that $\mathbf{a}_{*}=\mathbf{a}_{1}-\cdots-\mathbf{a}_{m}-\mathbf{a}_{1}$. This implies that every edge $\mathbf{v w} \in c(X)$ is included in exactly two triangles in $X$ (indeed, $\mathbf{w}$ is an $\mathbf{a}_{i}$ and all $\mathbf{a}_{i}$ are distinct).

According to [5,9], $X$ is a 2-manifold with boundary iff for every vertex $\mathbf{v} \in c(X)$, there is a non-empty set of edge(s) $\mathbf{a}_{*}$ such that $X_{\mathbf{v}}=\mathbf{v} \times \mathbf{a}_{*}$ with two cases: there are distinct vertices $\mathbf{a}_{i}$ such that $\mathbf{a}_{*}=\mathbf{a}_{1} \cdots \mathbf{a}_{m}$ or $\mathbf{a}_{*}=\mathbf{a}_{1} \cdots \mathbf{a}_{m}-\mathbf{a}_{1}$ is a cycle ( $m \geq 2$ in the first case and $m \geq 3$ in the second case). Thus every $\mathbf{a}_{i} \in c\left(X_{\mathbf{v}}\right)$ and every edge vw $\in c(X)$ is included in one or two triangle(s) in $X$ (but not more).

\subsection{Connectivity and genus}

Let $X \subseteq c\left(T^{\infty}\right)$. We say that $X$ is connected if for all vertices $\mathbf{v}$ and $\mathbf{v}^{\prime}$ in $c(X)$, there are vertices $\mathbf{v}_{i} \in$ $c(X)$ such that $1 \leq i \leq m, \mathbf{v}=\mathbf{v}_{1}, \mathbf{v}^{\prime}=\mathbf{v}_{m}$ and $\mathbf{v}_{1}-\mathbf{v}_{2}-\cdots-\mathbf{v}_{m} \subseteq c(X)$. A connected component $X$ is a connected subset of $X$ that is maximal in the inclusion sense. If two sets are homeomorphic, they have the same number of connected components. If $X \subseteq c(T), X$ is connected iff $|X|$ is connected in $\mathbb{R}^{3}$.

If $X$ is a set of simplices with a same dimension $k$, $X$ is strongly connected if for all $\sigma$ and $\sigma^{\prime}$ in $X$, there are $\sigma_{i} \in X$ such that $1 \leq i \leq m, \sigma_{1}=\sigma, \sigma_{m}=\sigma^{\prime}$ and every $\sigma_{i} \cap \sigma_{i+1}$ is a $k-1$ dimensional simplex. For example, $T^{\infty}, T$ and $\partial T$ are strongly connected. If $X$ is strongly connected, $X$ is connected.

Let $X \subset c(T)$ be a connected 2-manifold. The genus $g$ of $X$ meets $2(1-g)=v-e+t$ where $v, e$ and $t$ are the numbers of the vertices, edges and triangles in $c(X)$, respectively. Two connected 2-manifolds in $c(T)$ are homeomorphic iff they have the same genus. The genus is the number of handle(s) of $X$, e.g. $g=0$ if $X$ is a 2 -sphere, $g=1$ if $X$ is homeomorphic to a torus. The genus of a non-connected 2-manifold in $c(T)$ is the sum of genuses of its connected components. 


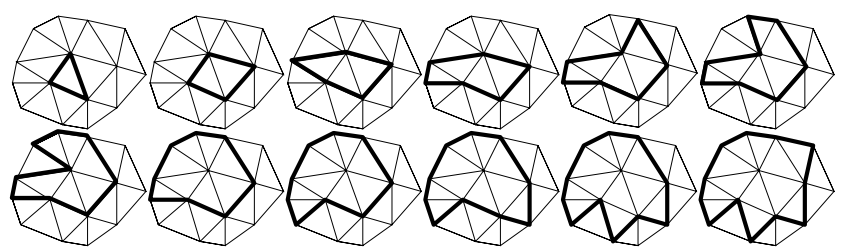

Fig. 1 A shelling example in the 2D case: $O$ is a growing set of triangles such that $\partial O$ is a cycle (drawn with bold edges).

\section{Shelling definition and properties}

First Sec. 3.1 provides our definition and explains the shelling algorithm in [13]. Then Sec. 3.2 describes the two cases of shelling steps. Last Sec. 3.3 details the topological changes in these two cases.

\subsection{Definition}

In our context (surface reconstruction), a shelling is a series of tetrahedron sets $O_{0}, O_{1}, \cdots, O_{n}$ such that

1. $O_{0} \subseteq T^{\infty}$ and $\partial O_{0}$ is a non-empty 2-manifold

2. $O_{i+1}=O_{i} \cup\left\{\Delta_{i}\right\}$ where a tetrahedron $\Delta_{i} \in T \backslash O_{i}$ and $c\left(O_{i}\right) \cap c\left(\Delta_{i}\right)$ is a non-empty 2D-pure simplicial complex if $0 \leq i<n$.

Thus every $\partial O_{i}$ is a 2 -manifold thanks to Theorem 1. Fig. 1 shows a shelling in the $2 \mathrm{D}$ case to help intuition: replace tetrahedra by triangles, 2-manifold by 1 manifold (a disjoint union of cycles), 2D-pure by 1Dpure.

There is another definition in remark 8.3.ii of [25] (see also remark 2 in chapter 7 of [9], and definition 2.7 of [12]): a shelling of a $d$-D pure simplicial complex $K$ is a series $\sigma_{0}, \sigma_{1}, \cdots, \sigma_{n}$ of $d$-D simplices such that $K=$ $c\left(\sigma_{0}, \sigma_{1}, \cdots \sigma_{n}\right)$ and every $c\left(\sigma_{0}, \sigma_{1}, \cdots \sigma_{j-1}\right) \cap c\left(\sigma_{j}\right)$ is a non-empty $(d-1)$-D-pure simplicial complex. Both definitions are equivalent if $d=3$ and $O_{0}=\left\{\sigma_{0}\right\} \subseteq T$ and $K \subseteq c(T)$.

Our definition is different by two ways. First we prefer a cumulative formulation based on tetrahedron sets $O_{i}$ since our application computes surfaces $\partial O_{i}$. Second a more general initialization $O_{0}$ is used since shellings are started at several steps of the surface reconstruction in [13]. Indeed,

Theorem 2 Algorithm 1 in [13] generates a shelling.

We describe this algorithm before the proof of Theorem 2. Let $F \subseteq T$ be the free-space: a tetrahedron $\Delta \in F$ iff there is a point $\mathbf{x}$ reconstructed from a view-point $\mathbf{c}$ using Computer Vision techniques such that $\Delta \cap \mathbf{c x} \neq \emptyset$. Let $n_{\Delta}$ be the number of line segments cx intersecting $\Delta$. The goal is the computation

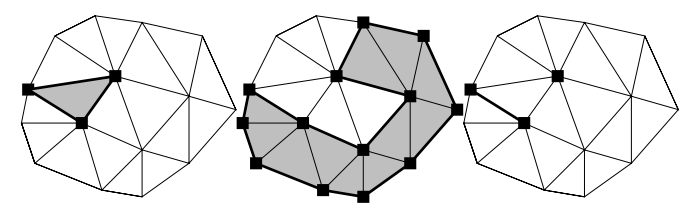

Fig. 2 Lemma 1 in the 2D case. Left: $c(\Delta)$ and $c(\partial \Delta)$. Middle: $c(O)$ and $c(\partial O)$. Right: $c(\Delta) \cap c(O)$. Both $\Delta$ and $O$ are in gray, $T$ is the set of all triangles, $\Delta \in T \backslash O$, the edges of $c(\partial \Delta)$ and $c(\partial O)$ are thickened, the vertices of $c(\Delta)$ and $c(O)$ are black squares. We see that $c(\Delta) \cap c(O)$ has 1 edge and 3 vertices, $c(\Delta) \cap c(O)=c(\partial \Delta) \cap c(\partial O), \partial O \cap \partial \Delta$ has 1 edge.

of $O \subseteq F$ that maximizes $\sum_{\Delta \in O} n_{\Delta}$ subject to the constraint that $\partial O$ is a 2 -manifold. Let $Q \subseteq F \backslash O$ be a set of tetrahedra that should be added to the current estimate of $O$. Furthermore, $Q$ is included in the immediate neighborhood of $O$ : every tetrahedron $\Delta \in Q$ has a common triangle with a tetrahedron in $O$ (if $O \neq \emptyset$ ), i.e. $\partial \Delta \cap \partial O \neq \emptyset$. At each step of Algorithm 1, we remove a tetrahedron $\Delta$ from $Q$ with the largest $n_{\Delta}$, then we add $\Delta$ to $O$ if $\partial O$ remains a 2 -manifold. If $\Delta$ is added to $O$, we also add to $Q$ every tetrahedron $\Delta^{\prime} \in F \backslash O$ such that $\partial \Delta \cap \partial \Delta^{\prime} \neq \emptyset$. This algorithm stops when $Q=\emptyset$. It is used several times in [13] for several initializations of $Q$ and $O$.

Proof This algorithm computes a series $O_{i}$ (consecutive values of $O$ ) such that $O_{i+1}=O_{i} \cup\left\{\Delta_{i}\right\}, \Delta_{i} \in T \backslash$ $O_{i}, \partial \Delta_{i} \cap \partial O_{i} \neq \emptyset$ and $\partial O_{i}$ is a 2-manifold for all $i$. According to Theorem $1, c\left(O_{i}\right) \cap c\left(\Delta_{i}\right)$ is $2 \mathrm{D}$ pure. Since $\partial \Delta_{i} \cap \partial O_{i} \subseteq c\left(O_{i}\right) \cap c\left(\Delta_{i}\right), c\left(O_{i}\right) \cap c\left(\Delta_{i}\right)$ is non-empty $2 \mathrm{D}$ pure. Furthermore $\partial O_{0}$ is a non-empty 2-manifold (if the algorithm starts with $O=\emptyset$, set $O_{0}$ as the first tetrahedron added to $O$ ).

\subsection{Basic properties}

Several remarks can be done about shelling thanks to the following Lemma (this is Lemma 1 in [14]).

Lemma 1 Let $O \subseteq T^{\infty}$ and $\Delta$ be a tetrahedron in $T \backslash O$. Then $c(O) \cap c(\Delta)$ is a simplicial complex in $\mathbb{R}^{3}$. Furthermore, $c(O) \cap c(\Delta)=c(\partial O) \cap c(\partial \Delta)$ and the triangles in $c(O) \cap c(\Delta)$ are exactly those in $\partial O \cap \partial \Delta$ (Fig. 2).

First the shelling definition is the same if we replace $c\left(O_{i}\right) \cap c\left(\Delta_{i}\right)$ by $c\left(\partial O_{i}\right) \cap c\left(\partial \Delta_{i}\right)$. Intuitively, this is the simplicial complex "between" $O_{i}$ and $\Delta_{i}$. Second the number of the triangles in $\partial O_{i} \cap \partial \Delta_{i}$ cannot be 0 .

Now we provide the two cases of shelling steps. They are in a corollary of the following theorem:

Theorem 3 Let $\Delta$ be a tetrahedron in T. Let $K \subseteq$ $c(\Delta)$ be a simplicial complex. Then $K$ is a non-empty 
2D-pure simplicial complex iff it is a 2-ball or a 2sphere. Let $f$ be the number of the triangles in $K$. In the 2-ball case, $f \in\{1,2,3\}$. In the 2-sphere case, $f=4$.

Proof We show that assertions $A$ " $K$ is a non-empty 2D-pure simplicial complex" and $B$ " $K$ is a 2-ball or a 2-sphere" are simultaneously true or false for every simplicial complex $K \subseteq c(\Delta)$.

If $K=\emptyset$ or $K=c(\Delta)$ or $K$ has only one vertex, both $A$ and $B$ are wrong. Otherwise $K$ has at least two vertices. If one of these vertices is not in an edge of $K$, $K$ is not connected. Thus both $A$ and $B$ are wrong.

Now we consider the case where an edge $\mathbf{a b} \in K$ and triangles abc $\notin K$ and abd $\notin K$ using notation abcd $=\Delta$. Here $A$ is wrong. Let points $\mathbf{x}=\frac{2}{3} \mathbf{a}+\frac{1}{3} \mathbf{b}$ and $\mathbf{y}=\frac{1}{3} \mathbf{a}+\frac{2}{3} \mathbf{b}$. Assume (reductio ad absurdum) that $B$ is true. Thus $|K| \backslash\{\mathbf{x}, \mathbf{y}\}$ is homeomorphic to a 2 ball or a 2-sphere minus two points, which is connected. This is impossible since $|K| \backslash\{\mathbf{x}, \mathbf{y}\}$ has (at least) two connected components: $\mathbf{x y} \backslash\{\mathbf{x}, \mathbf{y}\}$ and another one included in $(\mathbf{a x} \backslash\{\mathbf{x}\}) \cup(\mathbf{b y} \backslash\{\mathbf{y}\}) \cup \mathbf{c d a} \cup \mathbf{c d b}$.

Assume that we are not in the previous cases. Thus $A$ is true. We show that $B$ is true. We have triangles $t_{i} \in \partial \Delta$ and $K=c\left(t_{1}, t_{2}, \cdots, t_{f}\right)$ where $f \geq 1$. If $f=4$, $K=c(\partial \Delta)$ and $K$ is a 2-sphere. If $f=1,|K|=t_{1}$ is a 2-ball. If $f=3,|K|=t_{1} \cup t_{2} \cup t_{3}$ is homeomorphic to $t_{4}$ (use the piecewise linear function that is the identity at the $t_{4}$ vertices and maps the $t_{4}$ center to vertex $t_{1} \cap$ $\left.t_{2} \cap t_{3}\right)$. If $f=2,|K|=t_{1} \cup t_{2}$ is homeomorphic to $t_{3}$ (thanks to a similar piecewise linear function).

Corollary 1 A simplicial complex $c\left(O_{i}\right) \cap c\left(\Delta_{i}\right)$ is nonempty and 2D-pure (in the shelling definition) iff it is a 2-ball or a 2-sphere. Let $f$ be the number of the triangles in $\partial O_{i} \cap \partial \Delta_{i}$. In the ball case, $1 \leq f \leq 3$, otherwise $f=4$.

Proof The set $K=c\left(O_{i}\right) \cap c\left(\Delta_{i}\right)$ is a simplicial complex (Lemma 1) included in $c\left(\Delta_{i}\right)$. According to Theorem $3, K$ is non-empty and $2 \mathrm{D}$-pure iff it is a 2 -ball $(f \in\{1,2,3\})$ or a 2 -sphere $(f=4)$.

\subsection{Topology of surfaces $\partial O_{i}$}

Theorem 4 describes all topology change(s) by adding one tetrahedron in $O_{i}$. The two following lemmas are needed in its proof (the former is Lemma 3 in [14].

Lemma 2 Let $O \subset T^{\infty}$ and $\Delta$ be a tetrahedron in $T \backslash O$. Then $\partial(O \cup\{\Delta\})=(\partial \Delta \backslash \partial O) \cup(\partial O \backslash \partial \Delta)$.

Lemma 3 Let $O \subset T^{\infty}$ be such that $\partial O \subset c(T)$ and $\partial O$ is a 2-manifold. Let $\Delta$ be a tetrahedron in $T \backslash O$ such that $c(O) \cap c(\Delta)$ is $2 D$ pure. Let $f$ be the number of the triangles in $\partial O \cap \partial \Delta$. If $1 \leq f \leq 3, \partial O$ and $\partial(O \cup\{\Delta\})$ are homeomorphic.

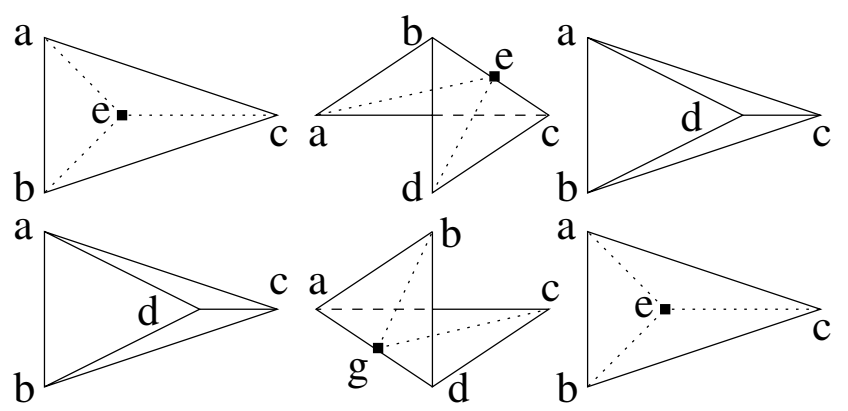

Fig. 3 Triangle splitting for Lemma 3 in cases $f=1$ (left), $f=2$ (middle) and $f=3$ (right). There are $\partial O \cap \partial \Delta$ on the top and $\partial \Delta \backslash \partial O$ on the bottom ( $\varphi$ maps the former to the latter).

Proof We find a homeomorphism $\varphi$ such that $\varphi(|\partial O|)=$ $|\partial(O \cup\{\Delta\})|$. First $\varphi$ is defined by its values on vertices and linear interpolation on the $\partial O$ triangles (or their subdivisions) as follows. For every vertex $\mathbf{v}$ in $c(\partial O)$, we set $\varphi(\mathbf{v})=\mathbf{v}$. We use notation $\Delta=\mathbf{a b c d}$.

If $f=1, \partial O \cap \partial \Delta=\{\mathbf{a b c}\}$, we split abc into triangles abe, bce, cae where $\mathbf{e}=(\mathbf{a}+\mathbf{b}+\mathbf{c}) / 3$, and set $\varphi(\mathbf{e})=\mathbf{d}$. We obtain $\varphi(\mathbf{a b c})=\mathbf{a b d} \cup \mathbf{b c d} \cup \mathbf{c a d}$.

If $f=2, \partial O \cap \partial \Delta=\{\mathbf{a b c}, \mathbf{b c d}\}$, we split bc into edges be and ec where $\mathbf{e}=(\mathbf{b}+\mathbf{c}) / 2$, we split $\mathbf{a d}$ into edges ag and $\mathbf{g d}$ where $\mathbf{g}=(\mathbf{a}+\mathbf{d}) / 2$, and set $\varphi(\mathbf{e})=\mathbf{g}$. Note that this scheme also splits every triangle including bc or ad into two other triangles. We obtain $\varphi(\mathbf{a b c} \cup \mathbf{b c d})=\mathbf{a d b} \cup$ adc.

If $f=3, \partial O \cap \partial \Delta=\{\mathbf{d a b}, \mathbf{d b c}, \mathbf{d c a}\}$, we split abc into triangles abe, bce, cae where $\mathbf{e}=(\mathbf{a}+\mathbf{b}+\mathbf{c}) / 3$, and reset $\varphi(\mathbf{d})=\mathbf{e}$. We obtain $\varphi(\mathbf{d a b} \cup \mathbf{d b c} \cup \mathbf{d c a})=$ abc. The three cases $f \in\{1,2,3\}$ are in Fig. 3 .

Second we check that every triangle $t \in \partial O \backslash \partial \Delta$ and $\varphi_{\mid t}(\varphi$ restricted to $t)$ are unchanged by the modifications above. If $t \cap \Delta=\emptyset$, both $t$ and $\varphi_{\mid t}$ are unchanged. Now we assume $t \cap \Delta \neq \emptyset$. Since $c(T)$ is a simplicial complex, $t \cap \Delta$ is a vertex or an edge of $\Delta$. We consider several cases. If $f=1, t \neq \mathbf{a b c}$ and $\mathbf{e}$ is not a vertex of $t$. Thus both $t$ and $\varphi_{\mid t}$ are unchanged. Assume (reductio ad absurdum) that $f=2$ and bc $\subset t$. Thus bc is a face of three distinct triangles $t$, abc and bcd in $\partial O$, although $\partial O$ is a 2 -manifold (impossible). Assume (reductio ad absurdum) that $f=2$ and ad $\subset t$. Thus ad $\in c(O) \cap c(\Delta)$ although triangles adb and adc are not in $c(O) \cap c(\Delta)$, i.e. $c(O) \cap c(\Delta)$ is not $2 \mathrm{D}$ pure (impossible). Therefore both $t$ and $\varphi_{\mid t}$ are unchanged if $f=2$. Assume (reductio ad absurdum) that $f=3$ and $\mathbf{d} \in t$. Thus $\{t\} \cup \mathbf{d} \times \mathbf{a}-\mathbf{b}-\mathbf{c}-\mathbf{a} \subseteq(\partial O)_{\mathbf{d}}$ and $t \notin \mathbf{d} \times \mathbf{a}-\mathbf{b}-\mathbf{c}-\mathbf{a}$, i.e. $\mathbf{d}$ is a singular vertex of $\partial O$ (impossible). Therefore both $t$ and $\varphi_{\mid t}$ are unchanged if $f=3$.

Third we show that $\varphi$ is surjective. Thanks to Lemma 2 and since $\varphi(|\partial O \cap \partial \Delta|)=|\partial \Delta \backslash \partial O|$ and $\varphi$ is the identity 


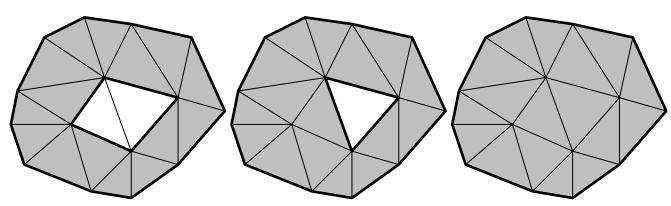

Fig. 4 Theorem 4 in the 2D case ( $O$ is gray). Left: $O$ encloses a cavity defined by two white triangles. Middle: add a triangle to $O$ such that $c(O) \cap c(\Delta)$ is a ball. Right: add a triangle to $O$ such that $c(O) \cap c(\Delta)$ is a sphere. The number of the connected component(s) of $\partial O$ is two (left and middle) or one (right).

$$
\begin{aligned}
& \text { in }|\partial O \backslash \partial \Delta|, \\
& \begin{aligned}
\varphi(|\partial O|) & =\varphi(|\partial O \cap \partial \Delta| \cup|\partial O \backslash \partial \Delta|) \\
& =|\partial \Delta \backslash \partial O| \cup|\partial O \backslash \partial \Delta| \\
& =|\partial(O \cup\{\Delta\})| .
\end{aligned}
\end{aligned}
$$

Fourth we show that $\varphi$ is a homeomorphism. We only need to check that $\varphi$ maps distinct vertices to distinct vertices [19]. This is true if $f=2$ and $f=3$. Assume (reductio ad absurdum) that $f=1$ and $\mathbf{d} \in$ $c(\partial O)$. Thus $\mathbf{d} \in c(O) \cap c(\Delta)$. Since triangles $\mathbf{d a b}, \mathbf{d b c}$ and dca are not in $c(O) \cap c(\Delta), c(O) \cap c(\Delta)$ is not 2D pure (impossible). If $f=1, \mathbf{d}=\varphi(\mathbf{e})$ is different to all vertices in $c(\partial O)$ and we obtain the result.

Note that Appendix B of [14] provides a first version of the proof of Lemma 3 without details on steps 2 and 4 .

Theorem 4 In a shelling, all $\partial O_{i}$ are 2-manifolds with the same genus. If $c\left(O_{i}\right) \cap c\left(\Delta_{i}\right)$ is a 2-ball, $\partial O_{i+1}$ and $\partial O_{i}$ have the same number of connected components. If $c\left(O_{i}\right) \cap c\left(\Delta_{i}\right)$ is a 2-sphere, $\partial O_{i}$ has one connected component more than $\partial O_{i+1}$ (Fig. 4).

Proof Every $\partial O_{i}$ is a 2-manifold thanks to Theorem 1 and since $\partial O_{0}$ is a 2-manifold. Let $f$ be the number of the triangles in $\partial O_{i} \cap \partial \Delta_{i}$. If $f \in\{1,2,3\}, \partial O_{i+1}$ and $\partial O_{i}$ are homeomorphic thanks to Lemma 3 . Therefore $\partial O_{i+1}$ and $\partial O_{i}$ have the same genus and the same number of connected component(s).

Now we study case $f=4$. We have $\partial \Delta_{i} \subseteq \partial O_{i}$ and Lemma 2 implies $\partial O_{i+1}=\partial O_{i} \backslash \partial \Delta_{i}$. Therefore $\partial O_{i}=\partial O_{i+1} \cup \partial \Delta_{i}$ and $\partial O_{i+1} \cap \partial \Delta_{i}=\emptyset$. Assume (reductio ad absurdum) that $\partial \Delta_{i}$ is not a connected component of $\partial O_{i}$. Thus these is an edge $\mathbf{v} \mathbf{v}^{\prime} \in c\left(\partial O_{i}\right)$ such that $\mathbf{v} \in c\left(\partial \Delta_{i}\right)$ and $\mathbf{v}^{\prime} \notin c\left(\partial \Delta_{i}\right)$. This implies that there is a triangle in $\partial O_{i} \backslash \partial \Delta_{i}$ that includes $\mathbf{v} \mathbf{v}^{\prime}$. Therefore $\mathbf{v} \in c\left(\partial \Delta_{i}\right) \cap c\left(\partial O_{i+1}\right)$. Since $\partial O_{i+1}$ is a 2 manifold, there is a cycle $\mathbf{a}_{*}$ such that $\left(\partial O_{i+1}\right)_{\mathbf{v}}=\mathbf{v} \times$ $\mathbf{a}_{*}$. Thus $\left(\mathbf{v} \times \mathbf{a}_{*}\right) \cup\left(\partial \Delta_{i}\right)_{\mathbf{v}} \subseteq\left(\partial O_{i}\right)_{\mathbf{v}}$ and $\left(\partial \Delta_{i}\right)_{\mathbf{v}} \backslash \mathbf{v} \times$ $\mathbf{a}_{*} \neq \emptyset$, which imply that $\mathbf{v}$ is a singular vertex of $\partial O_{i}$ (impossible). Since $\partial \Delta_{i}$ is a connected component of $\partial O_{i}$ and $\partial O_{i}=\partial O_{i+1} \cup \partial \Delta_{i}, \partial O_{i}$ has one connected component more than $\partial O_{i+1}$. Last $\partial O_{i+1}$ and $\partial O_{i}$ have the same genus since $\partial \Delta_{i}$ has zero genus.

In most cases, $c\left(O_{i}\right) \cap c\left(\Delta_{i}\right)$ is a 2-ball. In the few cases where $\Delta_{i}$ is a cavity of $O_{i}\left(O_{0}\right.$ can be like a piece of Swiss cheese that has cavities as in Fig. 4), $c\left(O_{i}\right) \cap c\left(\Delta_{i}\right)$ is a 2 -sphere.

Corollary 2 describes a frequent case where shelling does not change the topology of $\partial O_{i}$. We need

Lemma 4 If $\emptyset \neq O \subsetneq T^{\infty}, \partial O \neq \emptyset$.

The proof of Lemma 4 is straightforward ( $T^{\infty}$ is strongly connected) and is in the supplementary material.

Corollary 2 Assume that $O_{i} \subset T^{\infty}$ and $\partial O_{i}$ is a 2sphere in a shelling. If $i \leq j<n$ and $O_{j+1} \neq T^{\infty}$, $c\left(O_{j}\right) \cap c\left(\Delta_{j}\right)$ is a 2-ball and $\partial O_{j+1}$ is a 2-sphere.

Proof We assume (induction) that $\partial O_{j}$ is a 2-sphere (this is OK if $j=i$ ) and show that $c\left(O_{j}\right) \cap c\left(\Delta_{j}\right)$ is a 2-ball and $\partial O_{j+1}$ is a 2-sphere if $O_{j+1} \neq T^{\infty}$.

Assume (reductio ad absurdum) that $c\left(O_{j}\right) \cap c\left(\Delta_{j}\right)$ is a 2 -sphere. Since $\partial O_{j}$ has one connected component, Theorem 4 implies that $\partial O_{j+1}$ does not have a connected component, i.e. $\partial O_{j+1}=\emptyset$. Since $\emptyset \neq O_{j+1} \subsetneq$ $T^{\infty}$, this contradicts Lemma 4 .

Thus $c\left(O_{j}\right) \cap c\left(\Delta_{j}\right)$ is a 2-ball (Corollary 1) and $\partial O_{j+1}$ is a 2-manifold with the same genus and the same number of connected component than $\partial O_{j}$ (Theorem 4), i.e. $\partial O_{j+1}$ is a 2 -sphere.

\section{Overview of 3-ball shellability}

This section summarizes previous works in Combinatorial Topology. Here we would like to know if $O \subseteq T$ is shellable, i.e. if $O$ can be the end value of a shelling started from a single tetrahedron. First Sec. 4.1 describes and compare several kinds of shellability and non-shellability. Second Sec. 4.2 presents invariant numbers and a necessary condition for the $O$ shellability. Last Sec. 4.3 and 4.4 list positive and negative cases of shellability.

\subsection{Definitions}

According to Theorem 5 (supplementary material),

Theorem 5 Let $O \subseteq T$. Then $O$ is a 3-ball iff $\partial O$ is a 2-sphere.

assertions " $O$ is a 3 -ball" and " $\partial O$ is a 2-sphere" are equivalent in our context. Although the introduction of 3-balls seems useless, we mention it (and use it) since this formulation is often used in the bibliography. 
A tetrahedron set $O \subseteq T$ is shellable if there is a tetrahedron $\Delta \in T$ and a shelling $O_{0} \cdots O_{n}$ such that $O_{0}=\{\Delta\}$ and $O=O_{n}$. We also say that $O_{0} \cdots O_{n}$ is a shelling of $O$. Thanks to Corollary 2, we note that all $\partial O_{i}$ are 2-spheres and all $c\left(O_{i}\right) \cap c\left(\Delta_{i}\right)$ are 2-balls. Thus $O$ is a 3 -ball.

A 3-ball $O \subseteq T$ is extendably shellable if for every shellable $O^{\prime} \subset O$, there is a shelling $O_{0} \cdots O_{k}$ such that $O_{0}=O^{\prime}$ and $O_{k}=O$. In other words, every partial shelling of $O$ can be completed to reach $O$. Therefore $O$ is shellable if $O$ is extendably shellable.

Extendable shellability is a convenient case of shellability. If $O$ is extendably shellable and if a greedy algorithm constructs a shelling of $O$ by choosing successive tetrahedra in $O$ (e.g. Algorithm 1 in [13] using $F=O$ ), the algorithm always succeeds, i.e. its final value is always $O_{n}=O$. If $O$ is shellable but non-extendably shellable, the greedy algorithm can choose tetrahedra at the shelling beginning such that it "gets stuck". This means that there is $O_{i} \subsetneq O$ in the shelling computation such that $c\left(O_{i}\right) \cap c(\Delta)$ is never non-empty 2D-pure for every tetrahedron $\Delta$ tried by the algorithm (thus the algorithm stops). Thanks to Theorem 1, this means that $\partial\left(O_{i} \cup\{\Delta\}\right)$ is not a 2 -manifold (i.e. $\partial\left(O_{i} \cup\{\Delta\}\right)$ has a singular vertex) or $c\left(O_{i}\right) \cap c(\Delta)=\emptyset$.

A 3-ball $O \subseteq T$ is strongly non-shellable if $O$ has at least two tetrahedra and if for every tetrahedron $\Delta \in O$, $c(\Delta) \cap c(\partial O)$ is not a 2-ball. Here we note that $\Delta \in O$ in contrast to the shelling definition where $\Delta_{i} \notin O_{i}$. According to Proposition 2.4.iv in [24], $O$ is non-shellable if $O$ is strongly non-shellable (principle of the proof: if $O_{0} \cdots O_{n}$ is a shelling of $O$, we have $O=O_{n-1} \cup\{\Delta\}$, and see that $c(\partial O) \cap c(\Delta)$ is a 2-ball as the complement of 2-ball $c\left(\partial O_{n-1}\right) \cap c(\Delta)$ in 2-sphere $\left.c(\partial \Delta)\right)$.

Strongly non-shellability is an easy case of non-shellability for several reasons. First it is not difficult to check that a given $O$ is strongly non-shellable. In comparison, it is quite more difficult to check that $O$ is non-shellable: we don't know whether a polynomialtime algorithm can do that [8]. Second, a strongly nonshellable $O$ can be build with a few tetrahedra. Third, non-shellability and strongly non-shellability are related by Proposition 2.4.v in [24]: if $O$ is non-shellable, there is a strongly non-shellable 3 -ball $O^{\prime} \subseteq O$ (principle of the proof: construct a shelling of $O$ in the reverse order if every 3-ball $O^{\prime} \subseteq O$ is not strongly non-shellable). More details about the relations between strongly nonshellability and non-shellability are in the supplementary material.

\section{$4.2 h$-numbers of $O$}

The result described in this section is known for every dimension (Theorem 8.19 in [25], see also Proposition 7.7 in [9]). Here we present a simpler (specialized) proof in our $3 \mathrm{D}$ case.

Let $O$ be a 3 -ball in $T$ and $f_{0}$ (respectively, $f_{1}, f_{2}$ and $f_{3}$ ) be the number of the vertices (respectively, edges, triangles and tetrahedra) in $c(O)$. According to [25, $9]$, the definition of $h$-numbers $h_{1}, h_{2}$ and $h_{3}$ of $O$ is

$$
\left(\begin{array}{c}
1 \\
h_{1} \\
h_{2} \\
h_{3}
\end{array}\right)=\mathrm{H}\left(\begin{array}{c}
1 \\
f_{0} \\
f_{1} \\
f_{2}
\end{array}\right) \text { where } \mathrm{H}=\left(\begin{array}{cccc}
+1 & 0 & 0 & 0 \\
-4 & +1 & 0 & 0 \\
+6 & -3 & +1 & 0 \\
-4 & +3 & -2 & +1
\end{array}\right) .
$$

If $O$ is shellable, we classify the shelling steps in three cases and all steps in a case update the numbers of the simplices by the same way. Thanks to this, Theorem 6 shows that the number of the steps in the $j$-th case is a function of the $f_{i} \mathrm{~s}$, more precisely it is $h_{j}$. The number of the steps in the $j$-th case is invariant to the shelling choice since $h_{j}$ does not depend on it.

Theorem 6 If $O$ is shellable, every shelling of $O$ has $h_{j}$ step $(s) O_{i+1}=O_{i} \cup\left\{\Delta_{i}\right\}$ such that the number of the triangles in $\partial O_{i} \cap \partial \Delta_{i}$ is equal to $j$.

Proof If $X \subseteq c(T)$, we define the vector $f(X) \in \mathbb{N}^{4}$ whose $k$-th coordinate is the number of $k$-1-dimensional simplices in $c(X)$. Let $O_{0} \cdots O_{n}$ be a shelling of $O$ and the tetrahedron $\Delta_{i} \in T \backslash O_{i}$ such that $O_{i+1}=O_{i} \cup\left\{\Delta_{i}\right\}$. We will calculate $\delta_{i}=f\left(c\left(O_{i+1}\right)\right)-f\left(c\left(O_{i}\right)\right)$.

Since $c\left(O_{i+1}\right)=c\left(O_{i}\right) \cup\left(c\left(\Delta_{i}\right) \backslash c\left(O_{i}\right)\right), \delta_{i}=f\left(c\left(\Delta_{i}\right) \backslash\right.$ $\left.c\left(O_{i}\right)\right)$. Let $n_{i}$ be the number of the triangles in $\partial O_{i} \cap$ $\partial \Delta_{i}$ and abcd $=\Delta_{i}$. Since $c\left(O_{i}\right) \cap c\left(\Delta_{i}\right)$ is a 2-ball, there are three cases according to Corollary 1 .

If $n_{i}=1, c\left(O_{i}\right) \cap c\left(\Delta_{i}\right)=c(\mathbf{a b c})$. Thus $c\left(\Delta_{i}\right) \backslash$ $c\left(O_{i}\right)=\{\mathbf{d}, \mathbf{d a}, \mathbf{d b}, \mathbf{d c}, \mathbf{d a b}, \mathbf{d b c}, \mathbf{d c a}, \mathbf{a b c d}\}$. We obtain $\delta_{i}=\left(\begin{array}{llll}1 & 3 & 3 & 1\end{array}\right)^{T}$.

If $n_{i}=2, c\left(O_{i}\right) \cap c\left(\Delta_{i}\right)=c(\mathbf{a b c}, \mathbf{b c d})$. Thus $c\left(\Delta_{i}\right) \backslash$ $c\left(O_{i}\right)=\{\mathbf{a d}, \mathbf{a d b}, \mathbf{a d c}, \mathbf{a b c d}\}$. We obtain $\delta_{i}=\left(\begin{array}{llll}0 & 1 & 2 & 1\end{array}\right)^{T}$.

If $n_{i}=3, c\left(O_{i}\right) \cap c\left(\Delta_{i}\right)=c(\mathbf{a b c}, \mathbf{a c d}, \mathbf{a d b})$. Thus $c\left(\Delta_{i}\right) \backslash c\left(O_{i}\right)=\{\mathbf{b c d}, \mathbf{a b c d}\}$. We obtain $\delta_{i}=\left(\begin{array}{llll}0 & 0 & 1 & 1\end{array}\right)^{T}$.

Let $j \in\{1,2,3\}$ and $h_{j}^{\prime}$ be the number of the $\operatorname{step}(\mathrm{s})$ $O_{i+1}=O_{i} \cup\left\{\Delta_{i}\right\}$ such that $n_{i}=j$. Let

$A=\left(\begin{array}{llll}4 & 1 & 0 & 0 \\ 6 & 3 & 1 & 0 \\ 4 & 3 & 2 & 1 \\ 1 & 1 & 1 & 1\end{array}\right)$ and $B=\left(\begin{array}{cccc}0 & 1 & 0 & 0 \\ 0 & 0 & 1 & 0 \\ 0 & 0 & 0 & 1 \\ -1 & 1 & -1 & 1\end{array}\right)$.

Since $O_{0}$ has one tetrahedron, $f\left(c\left(O_{0}\right)\right)=\left(\begin{array}{lllll}4 & 6 & 4 & 1\end{array}\right)^{T}$. Now we have

$f(c(O))=f\left(c\left(O_{0}\right)\right)+\sum_{i=0}^{n-1} \delta_{i}=\mathrm{A}\left(1 h_{1}^{\prime} h_{2}^{\prime} h_{3}^{\prime}\right)^{\top}$. 
Furthermore, Eqs. 6 and 5 imply

$f_{0}-f_{1}+f_{2}-f_{3}=(1-11-1) f(c(O))=1$,

which in turn implies

$f(c(O))=\left(\begin{array}{llll}f_{0} & f_{1} & f_{2} & f_{3}\end{array}\right)^{\top}=\mathrm{B}\left(\begin{array}{llll}1 & f_{0} & f_{1} & f_{2}\end{array}\right)^{\top}$.

Thanks to Eqs. 6 and 8 and since $\mathrm{H}=\mathrm{A}^{-1} \mathrm{~B}$, we obtain

$\left(1 h_{1}^{\prime} h_{2}^{\prime} h_{3}^{\prime}\right)^{\top}=\mathrm{H}\left(1 f_{0} f_{1} f_{2}\right)^{\top}$.

Therefore $h_{j}^{\prime}=h_{j}$ for $j \in\{1,2,3\}$.

We also see that a necessary condition for shellability is $h_{1} \geq 0$ and $h_{2} \geq 0$ and $h_{3} \geq 0$ ( $O$ is non-shellable if there is $j$ such that $\left.h_{j}<0\right)$. Furthermore, the $h_{j}$ are consistent even if $O$ is non-shellable:

Property 1 If $O \subseteq T$ is a 3-ball, $1+h_{1}+h_{2}+h_{3}=f_{3}$.

Proof According to Eq. 4,

$$
\begin{aligned}
1+h_{1}+h_{2}+h_{3} & =\left(\begin{array}{llll}
1 & 1 & 1 & 1
\end{array}\right) \mathrm{H}\left(\begin{array}{lll}
1 & f_{0} f_{1} f_{2}
\end{array}\right)^{\top} \\
& =-1+f_{0}-f_{1}+f_{2} .
\end{aligned}
$$

Last we obtain the result thanks to Euler's relation for 3-balls [9], i.e. $f_{0}-f_{1}+f_{2}-f_{3}=1$.

\subsection{Positive cases}

First, all 3-balls with less than 9 vertices are extendably shellable and almost all 3-balls with 9 vertices are shellable [17] (there are only 29 non-shellable 3-balls over the 2451305 combinatorial 3-balls with 9 vertices). Second, every 3D Delaunay $T$ is a shellable 3 -ball [24,3]. Thus every $O \subseteq T$ such that $|O|$ is convex is shellable. Indeed, such an $O$ is the set of all tetrahedra of the 3D Delaunay triangulation defined by using only the vertices in $c(O)$. Third Sec. 5 shows that $O \subseteq T$ is shellable if $|O|$ is star-shaped. This generalizes the convex case above, since a convex set is star-shaped with respect to every point in the convex set.

\subsection{Negative cases}

There is a survey [24] of non-shellable 3-balls in a more general context than ours: some of them are constructed using a polytopal complex instead of a simplicial complex, i.e. the tetrahedra are replaced by convex hulls with $n \geq 4$ vertices likes cubes. Since we focus on 3 -balls included in a 3D Delaunay triangulation, they should be converted to analog examples in a 3D Delaunay. There are two kinds of examples: strongly non-shellable 3-balls defined by a small (ideally minimal) set of simplices, and non-shellable 3-balls defined by an important (not explicit) set of simplices and a global property. In the former, there is a 3-ball that has only 10 vertices and 21 tetrahedra (or 12 vertices and 25 tetrahedra). In the latter, there is a 3-ball called "Knotted hole ball" $(K)$; $K$ is non-shellable thanks to a property based on the knot theory: there is a knotted curve (a cycle of edges that cannot be included in a 2-sphere) with all edges on the boundary of $K$ except one edge that is in $K$.

There are other negative cases. A 3D Delaunay triangulation $T$ can be non-extendably shellable [24]. Thus a greedy shelling algorithm can fail and miss $O$ if $O \subseteq T$ and $|O|$ is convex. Last Sec. 6 provides a family of strongly non-shellable 3 -balls that are not difficult to visualize in $3 \mathrm{D}$. It includes the 3 -ball with 12 vertices and 25 tetrahedra in [24], which is in a 3D Delaunay triangulation.

\section{Star-shape shelling}

In Sec. 5, there are tetrahedron sets $O, O^{\prime}$ and a point $\mathbf{c} \in|O|$ such that $O \subsetneq O^{\prime} \subseteq T$ and both $O^{\prime}$ and $O$ are cstar-shaped. We show that there is a shelling $O_{0} \cdots O_{n}$ such that $O_{0}=O$ and $O_{n}=O^{\prime}$. Such a result is suggested in [7] if $O^{\prime}=T$ and $O$ has a single tetrahedron. Here we need a non-degenerate case: $\mathbf{c}$ is not in a plane including a triangle of $c(T)$.

\subsection{Summary}

The proof has three main steps. First Sec. 5.2 provides useful lemmas and a star-shape criterion for a set of tetrahedra included in $T$ (Theorem 7). Second Sec. 5.3 describes a visibility relation between two tetrahedra in $T$ with respect to a view point $\mathbf{c}$ : $\Delta$ is behind $\Delta^{\prime}$. Then Sec. 5.3 shows that there is a "front" tetrahedron in $O^{\prime} \backslash O$ for this relation (Theorem 8), i.e. this tetrahedron is not behind another one in $O^{\prime} \backslash O$. Third Sec. 5.4 shows that the front tetrahedron is added to $O$ such that we obtain a shelling step (Theorem 9) thanks to Theorems 7 and 8 and lemmas. Then Sec. 5.4 shows the existence of a shelling between $O$ and $O^{\prime}$ (Corollary 3).

Last Sec. 5.5 presents a qualitative argument based on Corollary 3 to explain why the first shelling in [13] does not have excessive blocking.

\subsection{Prerequisites}

We give definitions before lemmas and theorems. If a and $\mathbf{b}$ are distinct points in $\mathbb{R}^{3}$, we distinguish the line 


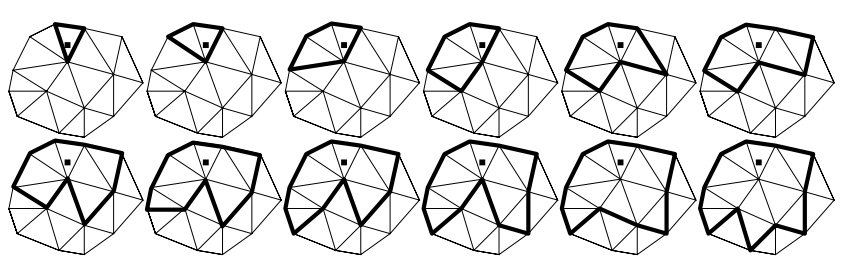

Fig. 5 A star-shape shelling example in the $2 \mathrm{D}$ case: $O$ is a growing set of triangles such that $\partial O$ is a cycle (bold edges) and $O$ is always star-shaped with respect to the black dot.

segment $\mathbf{a b}$ and the full line (ab) including $\mathbf{a}$ and $\mathbf{b}$ :

$$
\begin{aligned}
\mathbf{a b} & =\{(1-\lambda) \mathbf{a}+\lambda \mathbf{b}, 0 \leq \lambda \leq 1\} \text { and } \\
(\mathbf{a b}) & =\{(1-\lambda) \mathbf{a}+\lambda \mathbf{b}, \lambda \in \mathbb{R}\} .
\end{aligned}
$$

A point $\mathbf{d}$ is between $\mathbf{a}$ and $\mathbf{b}$ if $\mathbf{d} \in \mathbf{a b} \backslash\{\mathbf{a}, \mathbf{b}\}$.

A set $X \subset \mathbb{R}^{3}$ is star-shaped with respect to $\mathbf{c}$ (or $X$ is c-star-shaped) if $\mathbf{x} \in X$ implies that $\mathbf{x c} \subseteq X$. We say that $O \subseteq T$ is c-star-shaped if $|O|$ is c-star-shaped. If $t$ is a triangle in $\mathbb{R}^{3}$, the half-spaces of $t$ are the two half-spaces of $\mathbb{R}^{3}$ separated by the plane including $t$ (each half-space includes the plane itself). Note that our non degenerate case implies that $\mathbf{c}$ is in exactly one half-space of every triangle in $c(T)$. Fig. 5 shows a starshape shelling in the 2D case (replace tetrahedron by triangle etc, as in Fig. 1).

We also use other notations in Sec. 5. Let $\sigma$ be a simplex in $\mathbb{R}^{3}$ and $\stackrel{\circ}{\sigma}=\sigma \backslash|\partial \sigma|$, i.e. $\stackrel{\circ}{\sigma}$ is the interior of $\sigma$. Let $B(\mathbf{x}, \epsilon)$ be the $3 \mathrm{D}$ ball centered at $\mathbf{x} \in \mathbb{R}^{3}$ with the radius $\epsilon>0$. If $\sigma$ is a tetrahedron in $\mathbb{R}^{3}, \stackrel{\circ}{\sigma}$ is an open set in $\mathbb{R}^{3}$, i.e. every point $\mathbf{x} \in \stackrel{\circ}{\sigma}$ is such that there is $\epsilon>0$ and $B(\mathbf{x}, \epsilon) \subseteq \stackrel{\circ}{\circ}$.

We need the three following lemmas to show Theorem 7 (the star-shape criterion) and others. The first two help to escape from degenerate configurations. The third one is a ray-tracing lemma, which provides a series of adjacent tetrahedra in $T$ covering a line segment between two points in $|T|$.

Lemma 5 If a tetrahedron $\Delta \in T$ and a point $\mathbf{x} \in \dot{\Delta}$, $\Delta$ is the only tetrahedron in $T$ that contains $\mathbf{x}$, and $\mathbf{x}$ is not in a triangle of $c(T)$. If a triangle $t \in c(T)$ and $\mathbf{x} \in \dot{t}, t$ is the only triangle in $c(T)$ that contains $\mathbf{x}$.

The proof of Lemma 5 is straightforward $(c(T)$ is a simplicial complex) and is in the supplementary material.

Lemma 6 Let $A \subseteq T$ and points $\mathbf{c} \in|A|, \mathbf{x} \in|A| \backslash \mathbf{c}$, $\mathbf{y} \in \mathbf{c x}$ such that $\mathbf{y} \notin|A|$. Let $\Delta_{x}$ be a tetrahedron in $A$ such that $\mathbf{x} \in \Delta_{x}$. There is a point $\tilde{\mathbf{x}} \in \Delta_{x}$ and a tetrahedron $\Delta_{y} \in T \backslash A$ and a point $\tilde{\mathbf{y}} \in \Delta_{y} \cap \mathbf{c} \tilde{\mathbf{x}}$ such that $(\mathbf{c} \tilde{\mathbf{x}})$ does not intersect the edges in $c(T)$ (Fig. 6).

Lemma 7 Let $\Delta_{x}$ be a tetrahedron in $T$ and points $\mathbf{x} \in \stackrel{\circ}{x}_{x}, \mathbf{y} \in T \backslash\{\mathbf{x}\}$ such that $(\mathbf{x y})$ does not intersect
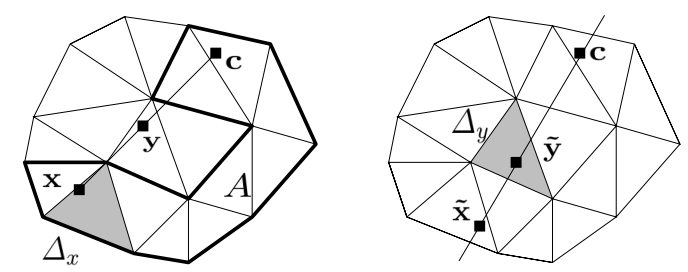

Fig. 6 Lemma 6 in the $2 \mathrm{D}$ case. Left: $A$ is enclosed by the bold edges, $\mathbf{c} \in|A|, \mathbf{x} \in \Delta_{x} \in A, \Delta_{x}$ is grey, (cx) intersects a vertex of $c(T)$ (an edge in $3 \mathrm{D}), \mathbf{y} \in \mathbf{c x} \backslash|A|$. Right: replace $\mathbf{x}$ by $\tilde{\mathbf{x}} \in \Delta_{x}$ such that $(\mathbf{c} \tilde{\mathbf{x}})$ does not intersect the $c(T)$ vertices, replace $\mathbf{y}$ by $\tilde{\mathbf{y}} \in \Delta_{y} \cap \mathbf{c} \tilde{\mathbf{x}}$ such that $\Delta_{y} \in T \backslash A, \Delta_{y}$ is grey.

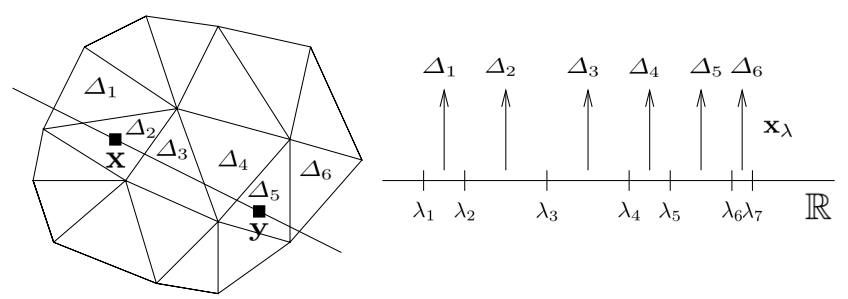

Fig. 7 Lemma 7 in the 2D case. Left: (xy) successively intersects $\Delta_{1} \cdots \Delta_{6}$ in $T$, every $\Delta_{i} \cap \Delta_{i+1}$ is an edge (a triangle in 3D). Right: $\mathbf{x}_{\lambda}$ maps $\left[\lambda_{i}, \lambda_{i+1}\right]$ in $\Delta_{i}$.
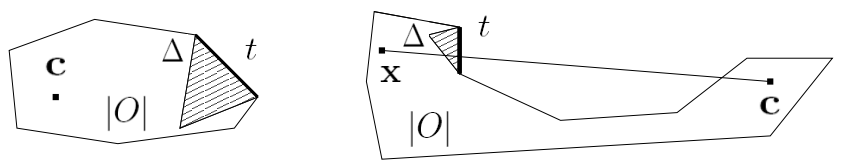

Fig. 8 Theorem 7 in the $2 \mathrm{D}$ case (tetrahedra are drawn as triangles). The streaked triangle is $\Delta$ and the bold edge is $t$. If $O$ is $\mathbf{c}$-star-shaped (left), $\Delta$ and $\mathbf{c}$ are in the same $t$ halfspace. If $O$ is not c-star-shaped (right), there is $\mathbf{x} \in|O|$ such that we have not $\mathbf{x c} \subset|O|$. Thus there are $\Delta$ and $t$ such that $\Delta$ and $\mathbf{c}$ are not in the same $t$ half-space.

the edges in $c(T)$. Let $\mathbf{x}_{\lambda}=(1-\lambda) \mathbf{x}+\lambda \mathbf{y}$ if $\lambda \in \mathbb{R}$. There are integer $k \geq 1$, reals $\lambda_{1} \cdots \lambda_{k+1}$, tetrahedra $\Delta_{1} \cdots \Delta_{k}$ in $T$ such that $\lambda_{i}<\lambda_{i+1}$ if $1 \leq i \leq k, \mathbf{x}_{\lambda} \in \Delta_{i}$ iff $\lambda \in\left[\lambda_{i}, \lambda_{i+1}\right]$, every $\Delta_{i} \cap \Delta_{i+1}$ is a triangle. If $\Delta \in T$

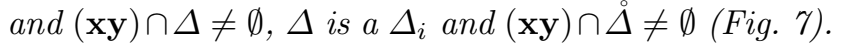

The proofs ot Lemma 6 and 7 are in Appendices A.1 and A.2). Theorem 7 converts the star-shape definition to a more tractable condition in our proof.

Theorem 7 Let $O \subseteq T$ and a point $\mathbf{c} \in|O|$. Then $O$ is $\mathbf{c}$-star-shaped iff every triangle $t \in \partial O$ is a face of a tetrahedron $\Delta \in O$ such that $\Delta$ and $\mathbf{c}$ are in the same t half-space (Fig. 8).

This is similar to star-shape tests in [20,22], which use outward-pointing normals at points on a volume boundary and a scalar product instead of our condition based on simplices. The proof is summarized in Fig. 8 and detailed in Appendix A.3. 
5.3 Visibility in a 3D Delaunay triangulation

Let tetrahedra $\Delta \in T$ and $\Delta^{\prime} \in T$. Let $l$ be a half-line starting at c. We say that $\Delta$ is behind [7] $\Delta^{\prime}$ if there is $l$ such that $l \cap \Delta \neq \emptyset$ and $l \cap \Delta^{\prime} \neq \emptyset$ and $\Delta \neq \Delta^{\prime}$ and every point in $(l \backslash\{\mathbf{c}\}) \cap \Delta^{\prime}$ is between $\mathbf{c}$ and every

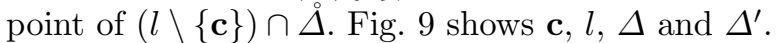

The goal of Sec. 5.3 is Theorem 8: the existence of a "front" tetrahedron in $O^{\prime} \backslash O$, i.e. a tetrahedron which is not behind another one in $O^{\prime} \backslash O$. The proof is based on the fact that the relation "is behind" is acyclic in the 3D Delaunay triangulation $T$ [7]. It also needs the two following lemmas to generate "behind" relations.

Lemma 8 Assume that $O^{\prime}$ is c-star-shaped and $\Delta \in$ $O^{\prime} \backslash O$ has a triangle face $t \notin \partial O$ such that $\Delta$ and $\mathbf{c}$ are not in the same $t$ half-space. There is $\Delta^{\prime} \in O^{\prime} \backslash O$ such that $\partial \Delta^{\prime} \cap \partial O \neq \emptyset$ and $\Delta$ is behind $\Delta^{\prime}$ (Fig. 9).

A sketch proof is provided before the proof for intuition. Let $\mathbf{y}$ be a point in $\Delta$. Since $O^{\prime}$ is $\mathbf{c}$-star-shaped, $\mathbf{y}+$ $\lambda(\mathbf{c}-\mathbf{y})$ successively intersects tetrahedra $\Delta_{0}, \Delta_{1} \cdots \Delta_{k}$ in $O^{\prime}$ when $\lambda$ increases from 0 to 1 , and every $\Delta_{i} \cap \Delta_{i+1}$ is not empty. We choose $\mathbf{y}$ such that $\Delta_{i} \cap \Delta_{i+1}$ is a triangle for every $i$ (cy does not intersect the edges in $c(T))$. Since $\mathbf{y} \in \Delta=\Delta_{0} \in O^{\prime} \backslash O$ and $\mathbf{c} \in \Delta_{k} \in O$, there is $i$ such that $\Delta_{i} \in O^{\prime} \backslash O$ and $\Delta_{i+1} \in O$. We obtain $\Delta^{\prime}=\Delta_{i}$ (we have $\Delta \neq \Delta^{\prime}$ if $\partial \Delta \cap \partial O=\emptyset$ ).

Proof First we set $A=O \cup\{\Delta\}$ and find points $\mathbf{x}^{\prime}$ and $\mathbf{y}^{\prime}$ that meet the assumptions of Lemma 6 . Let $\mathbf{x}^{\prime}$ be the barycentre of $t$ and $\Delta_{x}=\Delta \in A$. If $t \in \partial T$ (reductio ad absurdum), both $\Delta$ and $\mathbf{c}$ are in the same $t$ halfspace since $T$ is convex (impossible). Thus there is a tetrahedron $\Delta^{\prime \prime} \in T \backslash\{\Delta\}$ such that $t=\Delta \cap \Delta^{\prime \prime}$. Since $t \notin \partial O$ and $\Delta \notin O, \Delta^{\prime \prime} \in T \backslash O$ and thus $\Delta^{\prime \prime} \in T \backslash A$. Since $\mathbf{c}$ and $\Delta$ are in different half-spaces of $t$, there is a point $\mathbf{y}^{\prime} \in \mathbf{c x}^{\prime} \cap \Delta^{\prime \prime}$. Since $\mathbf{y}^{\prime}$ cannot be in a tetrahedron of $A$ (Lemma 5$), \mathbf{y}^{\prime} \notin|A|$.

Second we find a tetrahedron series covering $\mathbf{x c}(\mathbf{x} \in$ $\Delta)$ using Lemma 7. According to Lemma 6, there are a tetrahedron $\Delta_{y} \in T \backslash A$ and points $\mathbf{x} \in \Delta_{x}$ and $\mathbf{y} \in \Delta_{y} \cap \mathbf{c x}$ such that $(\mathbf{c x})$ does not intersect the edges in $c(T)$. Now we have a tetrahedron series $\Delta_{i} \in T$ that covers $|T| \cap(\mathbf{x y})$ as defined by Lemma 7 . Since xc $\subseteq|T|$ (indeed $|T|$ is convex and include both $\mathbf{x}$ and $\mathbf{c}$ ) and $(\mathbf{x c})=(\mathbf{x y})$, the tetrahedron series also covers $\mathbf{x c}$.

Third we find distinct $\Delta_{i}$ that include $\mathbf{x}, \mathbf{y}$ and $\mathbf{c}$. There are $j, n$ and $l$ such that $\Delta_{j}=\Delta_{x}, \Delta_{n}=\Delta_{y}$ and $\mathbf{c} \in \Delta_{l} \in O($ since $\mathbf{c} \in|O|)$. Since $\mathbf{y} \in \mathbf{x c}$ and $\mathbf{x}=\mathbf{x}_{0}$ and $\mathbf{y}=\mathbf{x}_{1}$, we have $j \leq n \leq l$. Since $\Delta_{j} \in A$ and $\Delta_{n} \notin A$ and $\Delta_{l} \in A$, we have $j<n<l$.

Fourth we show that $j \leq m \leq l$ implies $\Delta_{m} \in O^{\prime}$. Since $O^{\prime}$ is $\mathbf{c}$-star-shaped and both $\mathbf{x}$ and $\mathbf{c}$ are in $\left|O^{\prime}\right|$,

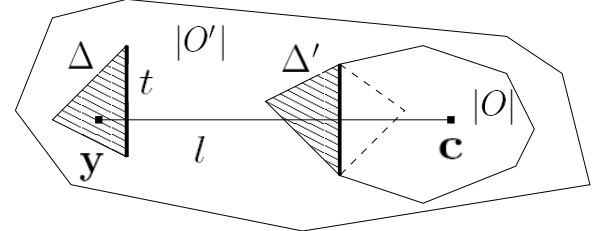

Fig. 9 Notations for Lemma 8 in the 2D case (tetrahedra are drawn as triangles). The two streaked triangles are $\Delta$ and $\Delta^{\prime}$, the triangle with dotted edges is in $O$. Here $\Delta$ is behind $\Delta^{\prime}$.
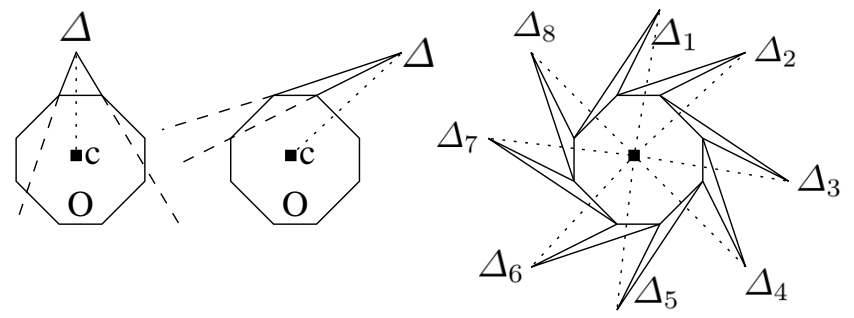

Fig. 10 Theorem 8 in the $2 \mathrm{D}$ case where $O$ forms an octagon that is c-star-shaped. Left: $\Delta$ meets all conditions of the theorem. Middle: $\Delta$ does not. Right: $\Delta$ never does (reductio ad absurdum). We see that $\Delta_{1}$ is behind $\Delta_{2}$, which is behind $\Delta_{3}$, ... which is behind $\Delta_{8}$, which is behind $\Delta_{1}$. However such a cycle is impossible in a Delaunay triangulation [7].

$\mathbf{c x} \in\left|O^{\prime}\right|$. Thus $\Delta_{m}^{\circ} \cap\left|O^{\prime}\right| \neq \emptyset($ Lemma 7$)$ and $\Delta_{m} \in O^{\prime}$ (Lemma 5).

Last we conclude. Since $\Delta_{n} \in O^{\prime} \backslash O$ and $\Delta_{l} \in O$, there is $m$ such that $\Delta_{m} \in O^{\prime} \backslash O$ and $\Delta_{m+1} \in O$ and $n \leq m<l$. Let $\Delta^{\prime}=\Delta_{m}$. The triangle $\Delta_{m} \cap \Delta_{m+1}$ is in $\partial \Delta^{\prime} \cap \partial O$. Note that $\mathbf{x}_{\lambda} \in \Delta$ iff $\lambda \in\left[\lambda_{j}, \lambda_{j+1}\right], \mathbf{x}_{\lambda} \in \Delta^{\prime}$ iff $\lambda \in\left[\lambda_{m}, \lambda_{m+1}\right], \lambda_{j+1} \leq \lambda_{m}$ (since $j<n \leq m$ ), $\mathbf{c}=\mathbf{x}_{\mu}$ with $\mu \in\left[\lambda_{l}, \lambda_{l+1}\right]$ and $\lambda_{m+1} \leq \lambda_{l}$ (since $m<l$ ). Thus $\Delta$ is behind $\Delta^{\prime}$.

Lemma 9 If $O^{\prime}$ is c-star-shaped and $O^{\prime} \backslash O \neq \emptyset$, there is a tetrahedron $\Delta^{\prime} \in O^{\prime} \backslash O$ such that $\partial \Delta^{\prime} \cap \partial O \neq \emptyset$.

Proof Let $\Delta$ be a tetrahedron in $O^{\prime} \backslash O$. If $\partial \Delta \cap \partial O \neq \emptyset$, we obtain $\Delta^{\prime}=\Delta$. Otherwise, $\partial \Delta \cap \partial O=\emptyset$. Note that $\mathbf{c} \notin \Delta(\mathbf{c} \notin \partial \Delta$ in our non degenerate case and $\mathbf{c} \notin \Delta$ by Lemma 5 and since $\mathbf{c} \in|O|)$. Thus there is a triangle $t \in \partial \Delta$ such that $\mathbf{c}$ and $\Delta$ are not in the same $t$ halfspace. Since $t \notin \partial O$, Lemma 8 provides $\Delta^{\prime}$.

Theorem 8 There is a tetrahedron $\Delta \in O^{\prime} \backslash O$ such that $\partial \Delta \cap \partial O \neq \emptyset$ and every triangle $t \in \partial \Delta \backslash \partial O$ is such that $\mathbf{c}$ and $\Delta$ are in the same $t$ half-space (Fig. 10).

Proof Assume (reductio ad absurdum) that

$\forall \Delta \in O^{\prime} \backslash O, \partial \Delta \cap \partial O=\emptyset$ or $\exists t \in \partial \Delta \backslash \partial O$ such that $\Delta$ and $\mathbf{c}$ are not in the same half-space of $t$. 
Thus $\Delta \in O^{\prime} \backslash O$ and $\partial \Delta \cap \partial O \neq \emptyset$ imply that $\Delta$ meets the assumptions of Lemma 8.

The principle of the proof is the following. We find an infinite series of tetrahedra $\Delta_{i} \in O^{\prime} \backslash O$ such that $\partial \Delta_{i} \cap \partial O \neq \emptyset$ and $\Delta_{i}$ is behind $\Delta_{i+1}$ for all $i$ using Lemma 8. Since $O^{\prime}$ is finite, there are $m \neq n$ such that $\Delta_{m}=\Delta_{n}$, i.e. the relation "is behind" has a cycle. This contradicts [7] which shows that this relation is acyclic in Delaunay triangulation $T$.

Here is the series. First Lemma 9 provides $\Delta_{0} \in$ $O^{\prime} \backslash O$ such that $\partial \Delta_{0} \cap \partial O \neq \emptyset$. Now $\Delta_{i+1}$ is defined from $\Delta_{i}$ as follow. If $\Delta_{i} \in O^{\prime} \backslash O$ and $\partial \Delta_{i} \cap \partial O \neq \emptyset, \Delta_{i}$ meets the assumptions of Lemma 8 (thanks to the reductio ad absurdum). Thus Lemma 8 provides $\Delta_{i+1} \in O^{\prime} \backslash O$ such that $\partial \Delta_{i+1} \cap \partial O \neq \emptyset$ and $\Delta_{i}$ is behind $\Delta_{i+1}$.

\subsection{Proof of star-shape shelling}

First we show in Theorem 9 that $c(O) \cap c(\Delta)$ is a 2-ball using the tetrahedron $\Delta$ provided by Theorem 8 . The proof highly relies on the fact that we are not in a degenerate case, i.e. $\mathbf{c}$ is not in a plane including a triangle of $c(T)$. It also requires the three following lemmas. Then a shelling between $O$ and $O^{\prime}$ is obtained in a corollary of Theorem 9.

Lemma 10 If $O$ is c-star-shaped and $\Delta$ is defined by Theorem 8, $O \cup\{\Delta\}$ is also c-star-shaped.

Proof According to Theorem 7 applied to $O$, every triangle $t \in \partial O \backslash \partial \Delta$ is a face of a tetrahedron $\Delta^{\prime} \in O$ such that $\Delta^{\prime}$ and $\mathbf{c}$ are in the same $t$ half-space. Theorem 8 implies that every triangle $t \in \partial \Delta \backslash \partial O$ is such that $\Delta$ and $\mathbf{c}$ are in the same $t$ half-space. Thanks to Lemma 2, every triangle $t \in \partial(O \cup\{\Delta\})$ is a face of a tetrahedron $\Delta^{\prime} \in O \cup\{\Delta\}$ such that $\Delta^{\prime}$ and $\mathbf{c}$ are in the same $t$ half-space. Last we apply Theorem 7 to $O \cup\{\Delta\}$ and see that $O \cup\{\Delta\}$ is c-star-shaped.

The proofs of the two following technical lemmas are in the supplementary material and Appendix B.

Lemma 11 Let abcd be a tetrahedron and $K$ be a simplicial complex such that $K \subseteq c(\mathbf{a b c d})$. If $c(\mathbf{a b c}) \subsetneq K$, $\mathbf{d} \in K$. If $c(\mathbf{a b c}, \mathbf{b c d}) \subsetneq K, \mathbf{a d} \in K$.

Lemma 12 Let $\Delta=\mathbf{v}_{1} \mathbf{v}_{2} \mathbf{v}_{3} \mathbf{v}_{4}$ and triangles $t_{i} \in \partial \Delta$ such that $\mathbf{v}_{i} \notin t_{i}$. Let $H_{i}$ and $H_{i}^{\prime}$ be the two half-spaces of $t_{i}$ such that $\Delta \subset H_{i}$. We have $H_{1}^{\prime} \cap H_{2}^{\prime} \cap H_{3}^{\prime} \cap H_{4}^{\prime}=\emptyset$. If $\mathbf{c} \in H_{1}^{\prime} \cap H_{2} \cap H_{3} \cap H_{4}, \mathbf{v}_{1} \mathbf{c} \cap t_{1} \neq \emptyset$. Let $\mathbf{v}=$ $\left(\mathbf{v}_{1}+\mathbf{v}_{2}\right) / 2$. If $\mathbf{c} \in H_{1}^{\prime} \cap H_{2}^{\prime} \cap H_{3} \cap H_{4}, \mathbf{v} \mathbf{c} \cap\left(t_{1} \cup t_{2}\right) \neq \emptyset$ (Fig. 11).

Theorem 9 If $O$ is c-star-shaped and $\Delta$ is defined by Theorem 8, $c(\partial \Delta) \cap c(\partial O)$ is a 2-ball.
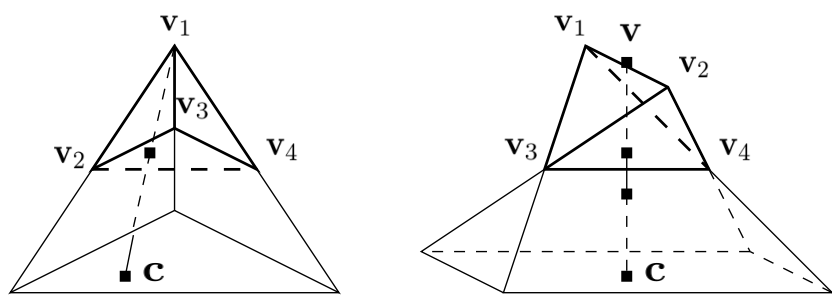

Fig. 11 The point vc $\cap t_{1}$ according to Lemma 12. We have $t_{1}=\mathbf{v}_{2} \mathbf{v}_{3} \mathbf{v}_{4}, t_{2}=\mathbf{v}_{3} \mathbf{v}_{4} \mathbf{v}_{1}, t_{3}=\mathbf{v}_{4} \mathbf{v}_{1} \mathbf{v}_{2}$ and $t_{4}=\mathbf{v}_{1} \mathbf{v}_{2} \mathbf{v}_{3}$. Left: $\mathbf{v}=\mathbf{v}_{1}$ and $\mathbf{c} \in H_{1}^{\prime} \cap H_{2} \cap H_{3} \cap H_{4}$. The half-spaces $H_{1}^{\prime}, H_{2}, H_{3}$ and $H_{4}$ are below $t_{1}, t_{2}, t_{3}$ and $t_{4}$ respectively. Right: $\mathbf{v}=\left(\mathbf{v}_{1}+\mathbf{v}_{2}\right) / 2$ and $\mathbf{c} \in H_{1}^{\prime} \cap H_{2}^{\prime} \cap H_{3} \cap H_{4}$. The half-spaces $H_{1}^{\prime}, H_{2}^{\prime}, H_{3}$ and $H_{4}$ are below $t_{1}, t_{2}, t_{3}$ and $t_{4}$ respectively.

Proof Let triangles $t_{i}$ be such that $\partial \Delta=\left\{t_{1}, t_{2}, t_{3}, t_{4}\right\}$ and $\partial \Delta \cap \partial O=\left\{t_{1}, \cdots, t_{n}\right\}$. Thanks to Theorem 8, $n \geq 1$. Let $K=c(\partial \Delta) \cap c(\partial O)$. We show that $K=$ $c\left(t_{1}, \cdots, t_{n}\right)$ where $1 \leq n \leq 3$ and conclude using Theorem 3. Note that $c\left(t_{1}, \cdots, t_{n}\right)=c(\partial \Delta \cap \partial O) \subseteq K$.

Let $H_{i}$ and $H_{i}^{\prime}$ be the two half-spaces of $t_{i}$ such that $\Delta \subset H_{i}$. First we show that $i \leq n$ implies $\mathbf{c} \in H_{i}^{\prime}$ and $i>n$ implies $\mathbf{c} \in H_{i}$. If $i \leq n$, there is a tetrahedron $\Delta_{i} \in O$ such that $t_{i}=\Delta \cap \Delta_{i}$ and $\Delta_{i} \subset H_{i}^{\prime}$. Since $O$ is $\mathbf{c}$-star-shaped, $\mathbf{c}$ and $\Delta_{i}$ are in the same $t_{i}$ half-space (Theorem 7), i.e. $\mathbf{c} \in H_{i}^{\prime}$. If $i>n, t_{i} \in \partial \Delta \backslash \partial O$. Thanks to Lemma $2, t_{i} \in \partial(O \cup\{\Delta\})$. Since $\partial(O \cup\{\Delta\})$ is cstar-shaped (Lemma 10), $\mathbf{c}$ and $\Delta$ are in the same $t_{i}$ half-space (Theorem 7 ), i.e. $\mathbf{c} \in H_{i}$.

Second we show that $n \neq 4$. If $n=4$ (reductio ad absurdum), $\mathbf{c} \in H_{1}^{\prime} \cap H_{2}^{\prime} \cap H_{3}^{\prime} \cap H_{4}^{\prime}$, which is impossible thanks to Lemma 12.

Third we show that $n=3$ implies $K=c\left(t_{1}, t_{2}, t_{3}\right)$. We have $c\left(t_{1}, t_{2}, t_{3}\right) \subseteq K \subseteq c(\partial \Delta)=c\left(t_{1}, t_{2}, t_{3}\right) \cup\left\{t_{4}\right\}$. If $c\left(t_{1}, t_{2}, t_{3}\right) \subsetneq K$ (reductio ad absurdum), $K=c(\partial \Delta)$, i.e. $n=4$ (impossible).

Fourth we show that $n=1$ implies $K=c\left(t_{1}\right)$. Thus $\mathbf{c} \in H_{1}^{\prime} \cap H_{2} \cap H_{3} \cap H_{4}$. Let $\mathbf{v}_{1}$ be the $\Delta$ vertex that is not in $t_{1}$, i.e. $\mathbf{v}_{1}=t_{2} \cap t_{3} \cap t_{4}$. Thanks to Lemma 12, there is a point $\mathbf{w} \in t_{1} \cap \mathbf{c v}_{1}$. Assume (reductio ad absurdum) that $c\left(t_{1}\right) \subsetneq K$. According to Lemma 11, $\mathbf{v}_{1} \in K \subseteq c(O)$. Since $O$ is c-star-shaped, $\mathbf{c v}_{1} \subset|O|$. We obtain $\mathbf{w v}_{1} \subseteq \Delta \cap|O|$. Since $\Delta \notin O$, Lemma 5 implies $\Delta \cap|O|=\emptyset$. Therefore $\mathbf{w v}_{1} \subset|\partial \Delta|$. There is a triangle $t_{i} \in \partial \Delta$ that includes at least two distinct points in $\mathbf{w} \mathbf{v}_{1}$. Since $\mathbf{c} \in\left(\mathbf{w} \mathbf{v}_{1}\right)$, $\mathbf{c}$ is in the $t_{i}$ plane: we are in a degenerate case (contradiction).

Last we show that $n=2$ implies $K=c\left(t_{1}, t_{2}\right)$. Thus $\mathbf{c} \in H_{1}^{\prime} \cap H_{2}^{\prime} \cap H_{3} \cap H_{4}$. Let $\mathbf{v}$ be the center of the edge $t_{3} \cap t_{4}$. Thanks to Lemma 12, there is a point $\mathbf{w} \in\left(t_{1} \cup t_{2}\right) \cap \mathbf{c v}$. Assume (reductio ad absurdum) that $c\left(t_{1}, t_{2}\right) \subsetneq K$. According to Lemma 11, $t_{3} \cap t_{4} \in K \subseteq$ $c(O)$ and $\mathbf{v} \in|O|$. Since $O$ is c-star-shaped, cv $\subset|O|$. 
Therefore wv $\subseteq \Delta \cap|O|$. Last we show that $\mathbf{c}$ is in a $t_{i}$ plane as in the previous case $n=1$ (contradiction).

Now we obtain the result of Sec. 5 .

Corollary 3 Let $\mathbf{c}$ be a point in $|T|$ that is not in a plane including a triangle of $c(T)$. Let $O \subseteq T$ such that $O$ is non-empty c-star-shaped. Then $\partial O$ is a 2manifold. Let $O^{\prime} \subseteq T$ such that $O \subsetneq O^{\prime}$ and $O^{\prime}$ is cstar-shaped. Then there is a shelling $O_{0} \cdots O_{n}$ such that $O_{0}=O, O_{n}=O^{\prime}$ and every $O_{i} \subseteq T$ is c-star-shaped.

Proof First we assume that both $\tilde{O}$ and $O_{i}$ are c-starshaped such that $\emptyset \neq O_{i} \subsetneq \tilde{O} \subseteq T$ and $\partial O_{i}$ is a 2manifold. We show that there is a tetrahedron $\Delta_{i} \in$ $\tilde{O} \backslash O_{i}$ such that $\partial \Delta_{i} \cap \partial O_{i} \neq \emptyset$ and, using notation $O_{i+1}=O_{i} \cup\left\{\Delta_{i}\right\}, O_{i+1}$ is c-star-shaped and $\partial O_{i+1}$ is a 2-manifold. Theorem 8 provides the tetrahedron $\Delta_{i}$. Let $O_{i+1}=O_{i} \cup\left\{\Delta_{i}\right\}$. Now $O_{i+1}$ is c-star-shaped (Lemma 10) and $c\left(\partial \Delta_{i}\right) \cap c\left(\partial O_{i}\right)$ is a 2-ball (Theorem 9). Thus $c\left(\Delta_{i}\right) \cap c\left(O_{i}\right)$ is non-empty 2D-pure (Theorem 3 ) and $\partial O_{i+1}$ is a 2 -manifold (Theorem 1 ).

Second we show that $\partial O$ is a 2 -manifold. Let $\Delta^{\prime}$ be a tetrahedron such that $\mathbf{c} \in \Delta^{\prime} \in T$. Let $O_{0}=\left\{\Delta^{\prime}\right\}$. Note that $O_{0}$ is c-star-shaped $\left(\Delta^{\prime}\right.$ is convex) and $\partial O_{0}$ is a 2-manifold ( $\partial O_{0}$ is a 2 -sphere). By successive use(s) of the first step (above) with $\tilde{O}=O$, we find a series $O_{0} \cdots O_{n}$ in $T$ such that $O_{n}=O$ and every $\partial O_{i}$ is a 2-manifold.

Third we show that there is a shelling $O_{0} \cdots O_{n}$ such that $O_{0}=O, O_{n}=O^{\prime}$ and every $O_{i}$ is c-star-shaped. We set $O_{0}=O$. Note that $O_{0}$ is c-star-shaped and $\partial O_{0}$ is a 2-manifold. By successive use(s) of the first step (above) with $\tilde{O}=O^{\prime}$, we find a series $O_{0} \cdots O_{n}$ in $T$ such that $O_{n}=O^{\prime}$ and every $O_{i}$ is c-star-shaped. This series is a shelling since $O_{i+1}=O_{i} \cup\left\{\Delta_{i}\right\}$ and $c\left(\Delta_{i}\right) \cap c\left(O_{i}\right)$ is non-empty 2D-pure for all $i<n$, and $\partial O_{0}$ is a non-empty 2 -manifold.

According to Appendix C, there are non-shellable starshapes in degenerate cases.

\subsection{A qualitative argument for surface reconstruction}

Here we provide a qualitative argument (this is not a proof as in the paper remainder) to explain that the first shelling in [13], i.e. Algorithm 1 started from $O=\emptyset$, does not have excessive blocking and provides most of the tetrahedra enclosed by the final surface. Sec. 3.1 gives a reminder of this algorithm and useful notations $F$ and $n_{\Delta}$.

The set $F$ looks like a star-shape: for every point $\mathbf{x}$ reconstructed from a camera location $\mathbf{c}, \mathbf{c x} \subset \cup F(F$ is not exactly a star-shape since $\mathbf{x}$ is restricted to the vertices in $c(T)$ and there are several c). Assuming that $\mathbf{c}$ follows a curve and the distance between $\mathbf{x}$ and $\mathbf{c}$ is small compared to the curve length, $F$ can be seen as a tubular neighborhood of the curve.

Furthermore, $O$ also looks like a star-shape during the first shelling in [13]. Since both $\Delta$ size and cx density increase when we go toward the curve, $n_{\Delta}$ increases when we go toward the curve. Since this shelling tries to add first to $O$ the tetrahedra $\Delta$ having the highest $n_{\Delta}$, $O$ essentially grows in a front-to-back order relatively to the curve. As a consequence, the points in $\cup F$ with a small distance to the curve enter in $\cup O$ during the shelling before those with a greater distance, i.e. there is a $\mathbf{c}$ in the curve such that $\mathbf{c x} \subset \cup O$ if $\mathbf{x} \in \cup O$.

Now $O$ is growing in $F$ by the shelling such that both $O$ and $F$ looks like star-shapes with the same curve as "center". Then Corollary 3 suggests that $O$ fills $F$, if we replace the point center by the curve center.

Let $O / F$ be the ratio of the number of the tetrahedra in $O$ generated by the first shelling alone and the number of the tetrahedra in $F$. Let $O / O_{e}$ be the ratio of the number of the tetrahedra in $O$ generated by the first shelling alone and the final number of the tetrahedra generated by all operators (including the first shelling). In Tab. 2 of [13], $O / F=87 \%$ and $O / O_{e}=94 \%$.

\section{Shelling blocking}

In this section, we provide a family of 3-balls that are strongly non-shellable with small numbers of simplices (Sec. 6.5). We also provide other cases of blocking (Sec. 6.6) for a shelling started from a general $O_{0} \subseteq T$. All these cases of shelling blocking are generated by disjoint unions of tetrahedron sets in $T$ that are called "pipe" (Sec. 6.1) and "slice" (Sec. 6.3). Both are 3-balls and their "gluing" (union of such a 3-ball with another tetrahedron set in $\left.T^{\infty}\right)$ are described in Secs. 6.2 and 6.4.

\subsection{Basic component: pipe}

If triangles $\mathbf{a}_{0} \mathbf{b}_{0} \mathbf{c}_{0}$ and $\mathbf{a}_{1} \mathbf{b}_{1} \mathbf{c}_{1}$ have distinct vertices, the tetrahedron set $P=p\left(\mathbf{a}_{0} \mathbf{b}_{0} \mathbf{c}_{0}, \mathbf{a}_{1} \mathbf{b}_{1} \mathbf{c}_{1}\right)$ is

$\left\{\mathbf{a}_{0} \mathbf{a}_{1} \mathbf{b}_{1} \mathbf{c}_{0}, \mathbf{a}_{0} \mathbf{a}_{1} \mathbf{b}_{0} \mathbf{c}_{0}, \mathbf{a}_{1} \mathbf{b}_{0} \mathbf{b}_{1} \mathbf{c}_{0}, \mathbf{a}_{0} \mathbf{a}_{1} \mathbf{b}_{1} \mathbf{c}_{1}, \mathbf{a}_{0} \mathbf{b}_{1} \mathbf{c}_{0} \mathbf{c}_{(}(\}\right)$

We assume that $P \subseteq T$, show $P$ in Fig. 12 , and see that $\mathbf{a}_{0} \mathbf{a}_{1} \mathbf{b}_{1} \mathbf{c}_{0}$ is an internal tetrahedron of $P$. Indeed, every triangle face of $\mathbf{a}_{0} \mathbf{a}_{1} \mathbf{b}_{1} \mathbf{c}_{0}$ is join to $\mathbf{b}_{0}$ or $\mathbf{c}_{1}$ by another tetrahedron in $P$ :

$$
\begin{aligned}
P=\left\{\mathbf{a}_{0} \mathbf{a}_{1} \mathbf{b}_{1} \mathbf{c}_{0}\right\} \cup \mathbf{b}_{0} & \times\left\{\mathbf{a}_{0} \mathbf{a}_{1} \mathbf{c}_{0}, \mathbf{a}_{1} \mathbf{b}_{1} \mathbf{c}_{0}\right\} \\
\cup & \mathbf{c}_{1} \times\left\{\mathbf{a}_{0} \mathbf{a}_{1} \mathbf{b}_{1}, \mathbf{a}_{0} \mathbf{b}_{1} \mathbf{c}_{0}\right\} .
\end{aligned}
$$




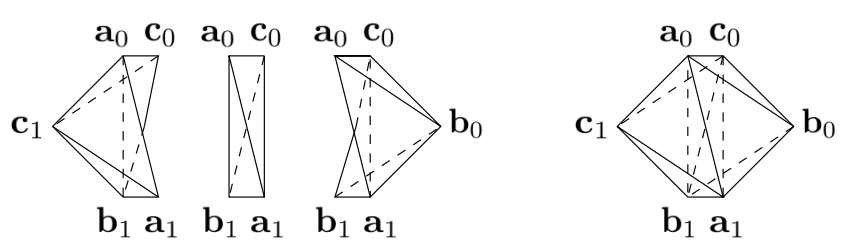

Fig. 12 The pipe $p\left(\mathbf{a}_{0} \mathbf{b}_{0} \mathbf{c}_{0}, \mathbf{a}_{1} \mathbf{b}_{1} \mathbf{c}_{1}\right)$ (right) and its decomposition (left). See the internal tetrahedron $\mathbf{a}_{0} \mathbf{a}_{1} \mathbf{b}_{1} \mathbf{c}_{0}$.

\section{Lemma 13 We have}

$$
\begin{aligned}
\partial P= & \left\{\mathbf{a}_{0} \mathbf{b}_{0} \mathbf{c}_{0}, \mathbf{a}_{1} \mathbf{b}_{1} \mathbf{c}_{1}\right\} \cup\left\{\mathbf{a}_{0} \mathbf{a}_{1} \mathbf{b}_{0}, \mathbf{a}_{1} \mathbf{b}_{0} \mathbf{b}_{1}\right\} \cup \\
& \left\{\mathbf{b}_{0} \mathbf{b}_{1} \mathbf{c}_{0}, \mathbf{b}_{1} \mathbf{c}_{0} \mathbf{c}_{1}\right\} \cup\left\{\mathbf{a}_{0} \mathbf{c}_{0} \mathbf{c}_{1}, \mathbf{a}_{0} \mathbf{a}_{1} \mathbf{c}_{1}\right\} .
\end{aligned}
$$

Proof Eq. 15 implies that the triangles in $c(P)$, that have neither $\mathbf{b}_{0}$ nor $\mathbf{c}_{1}$ as vertex, are faces of the internal tetrahedron $\mathbf{a}_{0} \mathbf{a}_{1} \mathbf{b}_{1} \mathbf{c}_{0}$. Since $\partial P \subset c(P)$, every triangle in $\partial P$ has vertex $\mathbf{b}_{0}$ or $\mathbf{c}_{1}$, i.e.

$$
\begin{aligned}
\partial P \subseteq & \mathbf{b}_{0} \times \mathbf{a}_{0}-\mathbf{a}_{1}-\mathbf{c}_{0}-\mathbf{a}_{0} \cup \mathbf{b}_{0} \times \mathbf{a}_{1}-\mathbf{b}_{1}-\mathbf{c}_{0}-\mathbf{a}_{1} \cup \\
& \mathbf{c}_{1} \times \mathbf{a}_{0}-\mathbf{a}_{1}-\mathbf{b}_{1}-\mathbf{a}_{0} \cup \mathbf{c}_{1} \times \mathbf{a}_{0}-\mathbf{b}_{1}-\mathbf{c}_{0}-\mathbf{a}_{0} .
\end{aligned}
$$

Since a triangle in $\partial P$ is in a single tetrahedron of $P$,

$$
\begin{aligned}
\partial P= & \mathbf{b}_{0} \times \mathbf{c}_{0}-\mathbf{a}_{0}-\mathbf{a}_{1} \cup \mathbf{b}_{0} \times \mathbf{a}_{1}-\mathbf{b}_{1}-\mathbf{c}_{0} \cup \\
& \mathbf{c}_{1} \times \mathbf{a}_{0}-\mathbf{a}_{1}-\mathbf{b}_{1} \cup \mathbf{c}_{1} \times \mathbf{b}_{1}-\mathbf{c}_{0}-\mathbf{a}_{0} \\
= & \mathbf{b}_{0} \times \mathbf{c}_{0}-\mathbf{a}_{0}-\mathbf{a}_{1}-\mathbf{b}_{1}-\mathbf{c}_{0} \cup \mathbf{c}_{1} \times \mathbf{a}_{0}-\mathbf{a}_{1}-\mathbf{b}_{1}-\mathbf{c}_{0}-\mathbf{a}_{0}
\end{aligned}
$$

and we obtain the result.

We say that $P$ is a pipe. Intuitively, the two cross sections of the pipe are triangles $\mathbf{a}_{0} \mathbf{b}_{0} \mathbf{c}_{0}$ and $\mathbf{a}_{1} \mathbf{b}_{1} \mathbf{c}_{1}$. The lateral section of the pipe is a strip of 6 triangles (in Eq. 16) having edges $\mathbf{a}_{0} \mathbf{a}_{1}, \mathbf{b}_{0} \mathbf{b}_{1}, \mathbf{c}_{0} \mathbf{c}_{1}$ and three others. A pipe series $p\left(\mathbf{a}_{0} \mathbf{b}_{0} \mathbf{c}_{0}, \mathbf{a}_{1} \mathbf{b}_{1} \mathbf{c}_{1}\right), p\left(\mathbf{a}_{1} \mathbf{b}_{1} \mathbf{c}_{1}, \mathbf{a}_{2} \mathbf{b}_{2} \mathbf{c}_{2}\right)$, $p\left(\mathbf{a}_{2} \mathbf{b}_{2} \mathbf{c}_{2}, \mathbf{a}_{3} \mathbf{b}_{3} \mathbf{c}_{3}\right), \cdots$ will be used to form a longer "pipe" and build strongly non-shellable 3-balls.

Lemma 14 If $p\left(\mathbf{a}_{0} \mathbf{b}_{0} \mathbf{c}_{0}, \mathbf{a}_{1} \mathbf{b}_{1} \mathbf{c}_{1}\right) \subseteq T, P$ is a 3-ball.

The proof ot this lemma is in the supplementary material (use a shelling, or see that $\partial P$ is an octahedron).

\subsection{Gluing a pipe}

The goal of Sec. 6.2 is Theorem 11: we define an union of a pipe $P$ and another tetrahedron set $A \subseteq T^{\infty}$ such that the criterion of the strongly non-shellability (Sec. 4.1) applied to $A \cup P$ is meet for every tetrahedron in $P$. The proof of this theorem (and others) needs the following gluing lemma and theorem.

Lemma 15 If $A \subseteq T^{\infty}$ and $B \subseteq T^{\infty}$ and $A \cap B=\emptyset$, $\partial(A \cup B)=(\partial A \backslash \partial B) \cup(\partial B \backslash \partial A)$.
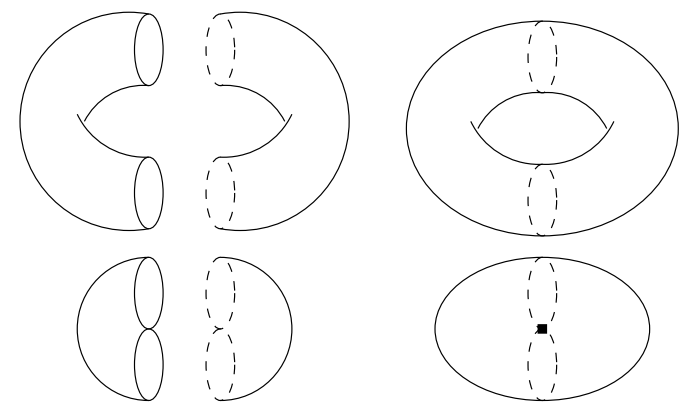

Fig. 13 Gluing $A \subset T$ (left) and $B \subset T$ (middle) using Theorem 10. Both $\partial A$ and $\partial B$ are 2-manifolds, $A \cap B=\emptyset$ and $\partial A \cap \partial B$ is the union of two 2-balls. Right: the result $A \cup B$. If $c(A) \cap c(B)$ is a 2-manifold with boundary, $\partial(A \cup B)$ is a 2-manifold (top). If $c(A) \cap c(B)$ is $2 \mathrm{D}$ pure and non-manifold, $\partial(A \cup B)$ can have a singular vertex (bottom).

Proof Let $^{1} t \in c\left(T^{\infty}\right)$ and $C=T^{\infty} \backslash(A \cup B)$. Let $\Delta$ and $\Delta^{\prime}$ be the two tetrahedra in $T^{\infty}$ such that $t=\Delta \cap \Delta^{\prime}$. We show that $t \in \partial(A \cup B)$ iff $t \in(\partial A \backslash \partial B) \cup(\partial B \backslash \partial A)$ in all cases.

If $\left(\Delta, \Delta^{\prime}\right) \in A \times A$, the triangle $t$ is neither in $\partial A \backslash$ $\partial B$ nor in $\partial(A \cup B)$. Since $A \cap B=\emptyset$, we also have $t \notin \partial B \backslash \partial A$. The case $\left(\Delta, \Delta^{\prime}\right) \in B \times B$ is similar. If $\left(\Delta, \Delta^{\prime}\right) \in C \times C, t$ is neither in $\partial(A \cup B)$ nor in $(\partial A \backslash \partial B) \cup(\partial B \backslash \partial A)$. If $\left(\Delta, \Delta^{\prime}\right) \in A \times B, t \notin \partial(A \cup B)$. Since $A \cap B=\emptyset, t \in \partial A \cap \partial B$ and thus $t \notin(\partial A)$ $\partial B) \cup(\partial B \backslash \partial A)$. If $\left(\Delta, \Delta^{\prime}\right) \in A \times C, t \in \partial A \backslash \partial B$ and $t \in \partial(A \cup B)$. The case $\left(\Delta, \Delta^{\prime}\right) \in B \times C$ is similar.

Theorem 10 Let $A \subseteq T^{\infty}$ and $B \subseteq T \backslash A$ such that both $\partial A$ and $\partial B$ are 2-manifolds, and $c(A) \cap c(B)$ is a 2manifold with boundary. Then $\partial(A \cup B)$ is a 2-manifold.

This theorem means that volumes $A$ and $B$ can be glued properly, i.e. by maintaining the 2 -manifold property of their boundaries, if their intersection is well behaved (Fig. 13). The proof is technical and is in Appendix D.

Theorem 11 Let $A \subseteq T^{\infty}$ such that $\partial A$ is a 2-manifold. Let $P=p\left(\mathbf{a}_{0} \mathbf{b}_{0} \mathbf{c}_{0}, \mathbf{a}_{1} \mathbf{b}_{1} \mathbf{c}_{1}\right)$ such that $P \subseteq T \backslash A$ and

$c(A) \cap c(P)=c\left(\mathbf{a}_{0} \mathbf{b}_{0} \mathbf{c}_{0}, \mathbf{a}_{1} \mathbf{b}_{1} \mathbf{c}_{1}, \mathbf{b}_{0} \mathbf{b}_{1} \mathbf{c}_{0}, \mathbf{b}_{1} \mathbf{c}_{0} \mathbf{c}_{1}\right)$.

Then $\partial(A \cup P)$ is a 2-manifold. If a tetrahedron $\Delta \in P$, $c(\partial(A \cup P)) \cap c(\Delta)$ is not a 2-ball (Fig. 14).

Proof Since the triangles in $c(A) \cap c(P)$ form a hexagon $\mathbf{a}_{0} \mathbf{b}_{0} \mathbf{b}_{1} \mathbf{a}_{1} \mathbf{c}_{1} \mathbf{c}_{0}, c(A) \cap c(P)$ is homeomorphic to a 2-ball. Then Lemma 14 and Theorem 10 imply that $\partial(A \cup P)$ is a 2-manifold.

We will show that $c(\partial(A \cup P)) \cap c(\Delta)$ has zero or one triangle and includes four vertices if $\Delta \in P$. This

1 Addendum: $t$ is a triangle. 

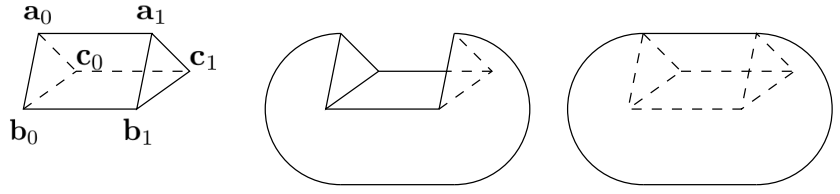

Fig. 14 Assumptions of Theorem 11: $P$ (left), $A$ (middle) and $A \cup P$ (right).

implies that $c(\partial(A \cup P)) \cap c(\Delta)$ is not 2D pure, which in turn implies that $c(\partial(A \cup P)) \cap c(\Delta)$ is not a 2-ball thanks to Theorem 3.

Since $\partial A \cap \partial P=\left\{\mathbf{a}_{0} \mathbf{b}_{0} \mathbf{c}_{0}, \mathbf{a}_{1} \mathbf{b}_{1} \mathbf{c}_{1}, \mathbf{b}_{0} \mathbf{b}_{1} \mathbf{c}_{0}, \mathbf{b}_{1} \mathbf{c}_{0} \mathbf{c}_{1}\right\}$, $\partial P \backslash \partial A=\left\{\mathbf{a}_{0} \mathbf{a}_{1} \mathbf{b}_{0}, \mathbf{a}_{1} \mathbf{b}_{0} \mathbf{b}_{1}, \mathbf{a}_{0} \mathbf{c}_{0} \mathbf{c}_{1}, \mathbf{a}_{0} \mathbf{a}_{1} \mathbf{c}_{1}\right\}$ (Eq. 16). Therefore

$\left\{\mathbf{a}_{0}, \mathbf{a}_{1}, \mathbf{b}_{0}, \mathbf{b}_{1}, \mathbf{c}_{0}, \mathbf{c}_{1}\right\} \subset c(\partial P \backslash \partial A)$

and

$$
\begin{aligned}
& \Delta=\mathbf{a}_{0} \mathbf{a}_{1} \mathbf{b}_{1} \mathbf{c}_{0} \Rightarrow \partial \Delta \cap(\partial P \backslash \partial A)=\emptyset \\
& \Delta=\mathbf{a}_{0} \mathbf{a}_{1} \mathbf{b}_{0} \mathbf{c}_{0} \Rightarrow \partial \Delta \cap(\partial P \backslash \partial A)=\left\{\mathbf{a}_{0} \mathbf{a}_{1} \mathbf{b}_{0}\right\} \\
& \Delta=\mathbf{a}_{1} \mathbf{b}_{0} \mathbf{b}_{1} \mathbf{c}_{0} \Rightarrow \partial \Delta \cap(\partial P \backslash \partial A)=\left\{\mathbf{a}_{1} \mathbf{b}_{0} \mathbf{b}_{1}\right\} \\
& \Delta=\mathbf{a}_{0} \mathbf{a}_{1} \mathbf{b}_{1} \mathbf{c}_{1} \Rightarrow \partial \Delta \cap(\partial P \backslash \partial A)=\left\{\mathbf{a}_{0} \mathbf{a}_{1} \mathbf{c}_{1}\right\} \\
& \Delta=\mathbf{a}_{0} \mathbf{b}_{1} \mathbf{c}_{0} \mathbf{c}_{1} \Rightarrow \partial \Delta \cap(\partial P \backslash \partial A)=\left\{\mathbf{a}_{0} \mathbf{c}_{0} \mathbf{c}_{1}\right\} .
\end{aligned}
$$

Since $\Delta \in P$ has its four vertices in $\left\{\mathbf{a}_{0}, \mathbf{a}_{1}, \mathbf{b}_{0}, \mathbf{b}_{1}, \mathbf{c}_{0}, \mathbf{c}_{1}\right\}$ and thanks to Eq. 20, $c(\partial P \backslash \partial A) \cap c(\Delta)$ includes four vertices. Furthermore, Eq. 21 implies that $(\partial P \backslash \partial A) \cap$ $(\partial \Delta)$ has zero or one triangle for every $\Delta \in P$.

Assume (reductio ad absurdum) that there is a triangle $t \in(\partial A \backslash \partial P) \cap \partial \Delta$. Thus $t \subseteq \Delta \in P$ and $t \subseteq \Delta^{\prime} \in A$. Since $A \cap P=\emptyset$ (Eq. 19), we have $\Delta^{\prime} \notin P$ and obtain $t \in \partial P$, which contradicts $t \in \partial A \backslash \partial P$.

Thanks to Lemma $15,(\partial P \backslash \partial A) \cup(\partial A \backslash \partial P)=\partial(A \cup$ $P)$. Therefore $c(\partial(A \cup P))$ includes the four vertices of $\Delta$ and has zero or one triangle of $\Delta$.

\subsection{Basic component: slice}

Let integer $n \geq 3$ and vertices $\mathbf{b}_{0}, \cdots \mathbf{b}_{n}$ and $\mathbf{c}_{0}, \cdots \mathbf{c}_{n}$ in $c(T)$ that are distinct, with exceptions $\mathbf{b}_{n}=\mathbf{b}_{0}$ and $\mathbf{c}_{n}=\mathbf{c}_{0}$. We have cycles $\mathbf{b}_{*}=\mathbf{b}_{0}-\mathbf{b}_{1}-\cdots \mathbf{b}_{n-1}-\mathbf{b}_{0}$ and $\mathbf{c}_{*}=\mathbf{c}_{0}-\mathbf{c}_{1}-\cdots \mathbf{c}_{n-1}-\mathbf{c}_{0}$. Let

$$
\begin{aligned}
N & =\bigcup_{0 \leq i<n} N_{i} \text { where } \\
N_{i} & =\left\{\mathbf{b}_{i} \mathbf{b}_{i+1} \mathbf{c}_{i}, \mathbf{b}_{i+1} \mathbf{c}_{i} \mathbf{c}_{i+1}\right\} \text { or } \\
N_{i} & =\left\{\mathbf{c}_{i+1} \mathbf{b}_{i} \mathbf{b}_{i+1}, \mathbf{c}_{i} \mathbf{c}_{i+1} \mathbf{b}_{i}\right\} .
\end{aligned}
$$

The set $N$ is a strip of triangles that form an annulus and we have $\partial N=\mathbf{b}_{*} \cup \mathbf{c}_{*}$. We say that $S \subseteq T$ is a slice if $S$ is a 3 -ball such that $N \subseteq \partial S$ (see Fig. 15).

Slice properties are in the following theorem:
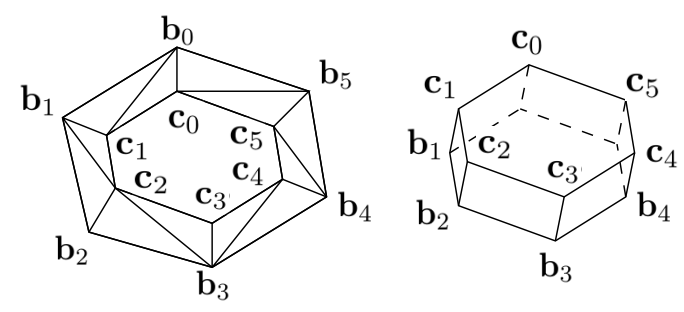

Fig. 15 Annulus (left) and slice (right) using $n=6$.

Theorem 12 Let $S$ be a slice with an annulus $N \subseteq$ $\partial S$. There are $B$ and $C$ such that $\partial S=N \cup B \cup C$, $\partial B=\mathbf{b}_{*}, \partial C=\mathbf{c}_{*}, c(B) \cap c(C)=\emptyset, c(B) \cap c(N)=$ $c\left(\mathbf{b}_{*}\right), c(C) \cap c(N)=c\left(\mathbf{c}_{*}\right)$, both $B$ and $C$ are strongly connected.

Intuitively, the boundary of the 3 -ball $S$ is a 2 -sphere segmented by $N$ in three connected parts $B, N, C$, whose common boundaries are $\mathbf{b}_{*}$ or $\mathbf{c}_{*}$. Furthermore, $S$ has two "sides" $|B|$ and $|C|$ which do not intersect. In the right of Fig. 15, $B$ is on the bottom and $C$ is on the top of the slice. The proof is technical (see Appendix E).

\subsection{Gluing a slice}

Similarly as in Sec. 6.2, the goal of Sec. 6.4 is Corollary 4: we define an union of a slice $S^{\prime}$ and another tetrahedron set $A \subseteq T^{\infty}$ such that the criterion of the strongly non-shellability applied to $A \cup S^{\prime}$ is meet for every tetrahedron in $S^{\prime}$. Here $S^{\prime}$ is computed by "shrinking" an initial slice $S$ : we progressively remove every tetrahedron $\Delta$ from $S$ that does not meet this criterion. The proof needs Theorem 13, which guarantees that $S^{\prime}$ is a slice (a 3 -ball with the same annulus as $S$ ). This theorem needs the following lemma.

Lemma 16 Let $A \subseteq T^{\infty}$ such that $\partial A$ is a 2-manifold. Let $S \subset T \backslash A$ be a 3-ball such that $c(A) \cap c(S)=c(N)$ and $N$ is an annulus. Then there are $B$ and $C$ as in Theorem 12 such that $c(\partial(A \cup S)) \cap c(S)=c(B) \cup c(C)$.

Proof Since $c(A) \cap c(S)=c(N)$ and $A \cap S=\emptyset$, every triangle in $N$ is in a tetrahedron in $A$ and another in $S$. Thus $S$ is a slice with the annulus $N \subset \partial S$. Let $B$ and $C$ as in Theorem 12. Now $N=\partial A \cap \partial S$ and Lemma 15 imply $\partial(A \cup S)=(\partial A \backslash N) \cup(\partial S \backslash N)$. We use shortened notation $\bar{S}=c(S)$. Since $\partial(A \cup S)=(\partial A \backslash N) \cup(\partial S \backslash N)$ and $\partial S \backslash N=B \cup C$ and $B \subset \bar{S}$ and $C \subset \bar{S}$,

$$
\begin{aligned}
c(\partial(A \cup S)) \cap \bar{S} & =(c(\partial A \backslash N) \cap \bar{S}) \cup(c(\partial S \backslash N) \cap \bar{S}) \\
& =(c(\partial A \backslash N) \cap \bar{S}) \cup c(B) \cup c(C) .
\end{aligned}
$$

Let $\sigma$ be a simplex in $c(\partial A \backslash N) \cap \bar{S}$ and show that $\sigma \in c(B) \cup c(C)$. Since $c(\partial A \backslash N) \cap \bar{S} \subseteq c(A) \cap \bar{S}=c(N)$, 
we have $\sigma \in c(N)$. We have $c(N)=c\left(\mathbf{b}_{*}\right) \cup c\left(\mathbf{c}_{*}\right) \cup \tilde{N}$ using

$\tilde{N}=\cup_{i} \tilde{N}_{i}$ where

$\tilde{N}_{i}=\left\{\mathbf{b}_{i} \mathbf{c}_{i}, \mathbf{b}_{i+1} \mathbf{c}_{i+1}, \mathbf{b}_{i+1} \mathbf{c}_{i}, \mathbf{b}_{i} \mathbf{b}_{i+1} \mathbf{c}_{i}, \mathbf{b}_{i+1} \mathbf{c}_{i} \mathbf{c}_{i+1}\right\}$ or

$\tilde{N}_{i}=\left\{\mathbf{b}_{i} \mathbf{c}_{i}, \mathbf{b}_{i+1} \mathbf{c}_{i+1}, \mathbf{b}_{i} \mathbf{c}_{i+1}, \mathbf{c}_{i+1} \mathbf{b}_{i} \mathbf{b}_{i+1}, \mathbf{c}_{i} \mathbf{c}_{i+1} \mathbf{b}_{i}\right\}(.24)$

If $\sigma \in c\left(\mathbf{b}_{*}\right) \cup c\left(\mathbf{c}_{*}\right), \sigma \in c(B) \cup c(C)$. Otherwise $\sigma \in \tilde{N}$. This implies that there is an edge $\mathbf{b}_{i} \mathbf{c}_{i}$ or $\mathbf{b}_{i+1} \mathbf{c}_{i}$ or $\mathbf{c}_{i+1} \mathbf{b}_{i}$ in $c(\partial(A \cup S)) \cap c(N)$. Let $e$ be this edge. There are distinct triangles $t_{1}$ and $t_{2}$ in $N=\partial S \cap \partial A$ such that $e=t_{1} \cap t_{2}$. Since $e \in c(\partial(A \cup S))=c((\partial A \cup \partial S) \backslash N)$, there is another triangle $t_{3} \in(\partial A \cup \partial S) \backslash N$ such that $e \subset t_{3}$. If $t_{3} \in \partial A, e$ is in 3 distinct triangles in $\partial A$. This is impossible since $\partial A$ is a 2-manifold. If $t_{3} \in \partial S, e$ is in 3 distinct triangles in $\partial S$. This is impossible since $\partial S$ is a 2-manifold.

Theorem 13 Let $A \subseteq T^{\infty}$ such that $\partial A$ is a 2-manifold. Let $S \subset T \backslash A$ be a 3-ball such that $c(A) \cap c(S)=c(N)$ and $N$ is an annulus. Let $\Delta$ be a tetrahedron in $S$ such that $c(\partial(A \cup S)) \cap c(\Delta)$ is a 2-ball. Then $S \backslash\{\Delta\}$ is a 3-ball and $c(A) \cap c(S \backslash\{\Delta\})=c(N)$.

Proof Let $B$ and $C$ be as in Lemma 16. Let $X=c(\Delta) \cap$ $c(B)$ and $Y=c(\Delta) \cap c(C)$. First we show that $X=\emptyset$ (or similarly, $Y=\emptyset$ ). Since $\Delta \in S$,

$$
\begin{aligned}
c(\Delta) \cap c(\partial(A \cup S)) & =c(\Delta) \cap(c(\partial(A \cup S)) \cap c(S)) \\
& =X \cup Y .
\end{aligned}
$$

Assume (reductio ad absurdum) that $X \neq \emptyset$ and $Y \neq \emptyset$. Since $c(B) \cap c(C)=\emptyset, X \cap Y=\emptyset$ and $c(\Delta) \cap c(\partial(A \cup S))$ is not connected. This contradicts that $c(\Delta) \cap c(\partial(A \cup$ $S)$ ) is a 2-ball.

Second we show that $c(\Delta) \cap c(N) \subseteq c(\Delta) \cap c\left(\mathbf{c}_{*}\right)$. Since $c(\Delta) \cap c\left(\mathbf{b}_{*}\right) \subseteq X=\emptyset, \Delta$ does not have a vertex in $c\left(\mathbf{b}_{*}\right)$. Since every vertex in $c(\Delta) \cap c(N)$ is a $\mathbf{b}_{i}$ or a $\mathbf{c}_{i}$, every vertex in $c(\Delta) \cap c(N)$ is a $\mathbf{c}_{i}$. Thus $c(\Delta) \cap c(N) \subseteq$ $c\left(\mathbf{c}_{*}\right)$. We obtain $c(\Delta) \cap c(N) \subseteq c(\Delta) \cap c\left(\mathbf{c}_{*}\right)$.

Third we show that $S \backslash\{\Delta\}$ is a 3-ball. Since $\partial S=$ $B \cup C \cup N$ and $c(\Delta) \cap c(N) \subseteq c(\Delta) \cap c\left(\mathbf{c}_{*}\right) \subseteq c(\Delta) \cap$ $c(C)=Y$,

$$
\begin{aligned}
c(\Delta) \cap c(\partial S) & =X \cup Y \cup(c(\Delta) \cap c(N))=Y \\
& =c(\Delta) \cap c(\partial(A \cup S)) .
\end{aligned}
$$

Therefore $c(\Delta) \cap c(\partial S)$ is a 2-ball. Since $S$ is a 3 -ball, we see that $S \backslash\{\Delta\}$ is a 3-ball (use Corollary 2 for the shelling $O_{0}=T^{\infty} \backslash S, O_{1}=O_{0} \cup\{\Delta\}$ and Theorem 5).

Last we show that $c(A) \cap c(S)=c(A) \cap c(S \backslash\{\Delta\})$. We have

$c(A) \cap c(S)=(c(A) \cap c(S \backslash\{\Delta\})) \cup(c(A) \cap c(\Delta))$.

Since $\Delta \in S$ and $c(N)=c(A) \cap c(S)$,

$c(A) \cap c(\Delta)=c(N) \cap c(\Delta) \subseteq c(\Delta) \cap c\left(\mathbf{c}_{*}\right)$.
Let $\sigma$ be a simplex in $c(A) \cap c(\Delta)$. Thanks to Eq. 28, $\sigma$ is a vertex or an edge. Since $c(N)=c(A) \cap c(S)$ and thanks to Eq. 27, $\sigma$ is in a triangle $t \in N$. Since $c(A) \cap$ $c(\Delta)$ does not have a triangle, $t \in c(A) \cap c(S \backslash\{\Delta\})$. Thus $c(A) \cap c(\Delta) \subseteq c(A) \cap c(S \backslash\{\Delta\})$.

Corollary 4 Let $A \subseteq T^{\infty}$ such that $\partial A$ is a 2-manifold. Let $S \subset T \backslash A$ be a 3-ball such that $c(A) \cap c(S)=c(N)$ and $N$ is an annulus. There is a 3-ball $S^{\prime} \subseteq S$ such that $c(A) \cap c\left(S^{\prime}\right)=c(N)$ and $c(\Delta) \cap c\left(\partial\left(A \cup S^{\prime}\right)\right)$ is not a 2-ball for every tetrahedron $\Delta \in S^{\prime}$.

Proof Let $S^{\prime}=S$. We consider the following loopalgorithm: while there is a tetrahedron $\Delta \in S^{\prime}$ such that $c(\Delta) \cap c\left(\partial\left(A \cup S^{\prime}\right)\right)$ is a 2-ball, remove $\Delta$ from $S^{\prime}$. Thanks to Theorem $13, S^{\prime}$ is always a 3 -ball and we always have $c(A) \cap c\left(S^{\prime}\right)=c(N)$. The algorithm stops since $S$ is finite, and it stops if $c(\Delta) \cap c\left(\partial\left(A \cup S^{\prime}\right)\right)$ is not a 2-ball for every tetrahedron $\Delta \in S^{\prime}$.

\subsection{Family of strongly non-shellable 3-balls}

First we need a specialization of Theorem 10 (proof in Appendix F):

Theorem 14 Let $A \subseteq T^{\infty}$ and $B \subseteq T \backslash A$ such that $\partial A$ is a connected 2-manifold, $\partial B$ is a 2-sphere, $c(A) \cap c(B)$ is a 2-ball. Then $\partial(A \cup B)$ is homeomorphic to $\partial A$.

Then Theorem 15 presents a family of strongly nonshellable 3-balls.

Theorem 15 Let $P_{i}=p\left(\mathbf{a}_{i} \mathbf{b}_{i} \mathbf{c}_{i}, \mathbf{a}_{i+1} \mathbf{b}_{i+1} \mathbf{c}_{i+1}\right)$ or $P_{i}=$ $p\left(\mathbf{a}_{i+1} \mathbf{b}_{i+1} \mathbf{c}_{i+1}, \mathbf{a}_{i} \mathbf{b}_{i} \mathbf{c}_{i}\right)$ such that $P_{i} \subseteq T$ if $0 \leq i<n$. Let $S \subseteq T \backslash \cup_{i} P_{i}$ be a 3-ball such that $c\left(\cup_{i} P_{i}\right) \cap c(S)=$ $c(N)$ and $N$ is an annulus using notations in Eq. 22 (top of Fig. 16). Then there is a 3-ball $S^{\prime} \subseteq S$ such that $c\left(\cup_{i} P_{i}\right) \cap c\left(S^{\prime}\right)=c(N)$ and $\cup_{i} P_{i} \cup S^{\prime}$ is a strongly non-shellable 3-ball (bottom of Fig. 16).

Intuitively (Fig. 16), $\partial\left(\cup_{i} P_{i}\right)$ is a torus whose intersection with $\partial S$ (and also $\partial S^{\prime}$ ) is the annulus $N$. Furthermore, $\partial\left(\cup_{i} P_{i} \cup S^{\prime}\right)$ is a 2 -sphere, with both "sides" separated by the cycle $\mathbf{a}_{*}$ ( $B$ is in one side and $C$ is in the other). If a tetrahedron $\Delta$ having a triangle in one side is removed from $\cup_{i} P_{i} \cup S^{\prime}$, a singular vertex appears in both sides. We use Theorem 11 if $\Delta \in P_{i}$ and Corollary 4 if $\Delta \in S^{\prime}$ to show that $c(\Delta) \cap c\left(\partial\left(\cup_{i} P_{i} \cup S^{\prime}\right)\right)$ is not a 2-ball, i.e. to show that $\cup_{i} P_{i} \cup S^{\prime}$ is a strongly non-shellable 3-ball.

Proof First we find $S^{\prime}$. Every $\partial P_{i}$ is a 2-manifold and every $c\left(\partial\left(\cup_{k=0}^{i-1} P_{k}\right)\right) \cap c\left(\partial P_{i}\right)$ is a 2 -manifold with boundary $\left(\left\{\mathbf{a}_{i} \mathbf{b}_{i} \mathbf{c}_{i}\right\}\right.$ if $i<n-1,\left\{\mathbf{a}_{n-1} \mathbf{b}_{n-1} \mathbf{c}_{n-1}, \mathbf{a}_{0} \mathbf{b}_{0} \mathbf{c}_{0}\right\}$ if $i=n-1)$. Thus successive uses of Theorem 10 imply that $\partial\left(\cup_{i=0}^{n-1} P_{i}\right)$ is a 2 -manifold. Now we use Corollary 4 

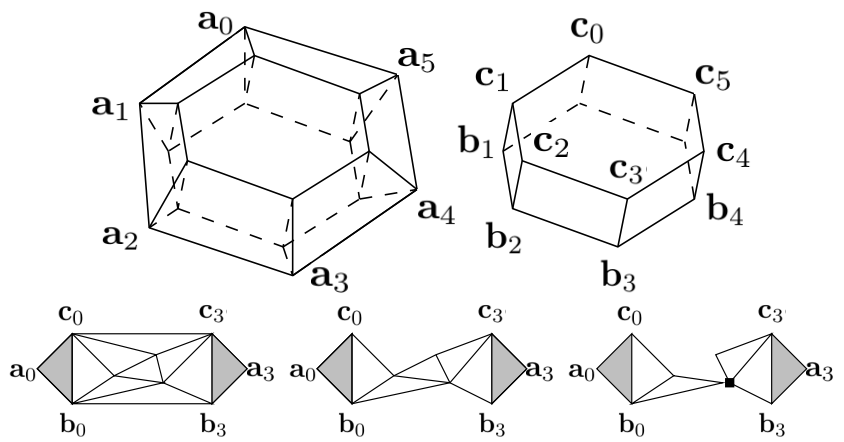

Fig. 16 Illustrations for Theorem 15. Top: $S$ (right) and $\cup_{i} P_{i} \cup S$ (left). The pipes $P_{i}$ form a torus; we have $c\left(\cup_{i} P_{i}\right) \cap$ $c(S)=c(N)$ where $N$ is the annulus whose boundary is $\mathbf{b}_{*} \cup$ $\mathbf{c}_{*}$. Bottom: cross section by a plane including grey triangles $\mathbf{a}_{0} \mathbf{b}_{0} \mathbf{c}_{0}$ and $\mathbf{a}_{3} \mathbf{b}_{3} \mathbf{c}_{3}$. The white triangles are tetrahedra in $S$ (left), $S^{\prime}$ is obtained by removing some of these tetrahedra such that $c\left(\cup_{i} P_{i}\right) \cap c\left(S^{\prime}\right)=c(N)$ (middle), a singular vertex appears if we remove a tetrahedron from $S^{\prime}$ (right).

using $A=\cup_{i} P_{i}$ and obtain a 3-ball $S^{\prime} \subseteq S$ such that $c(A) \cap c\left(S^{\prime}\right)=c(N)$ and $c(\Delta) \cap c\left(\partial\left(A \cup S^{\prime}\right)\right)$ is not a 2-ball for every tetrahedron $\Delta \in S^{\prime}$.

Second we show that $A_{i}=\cup_{j \neq i} P_{j} \cup S^{\prime}$ and $O=$ $\cup_{j} P_{j} \cup S^{\prime}$ are 3 -balls. By successive uses of Theorem 14 (as in the previous case above), $\partial\left(\cup_{j \neq i} P_{j}\right)$ is a 2 -manifold that is homeomorphic to a 2 -sphere. Then Theorem 14 implies that $\partial A_{i}$ is a 2 -sphere (use $A=\cup_{j \neq i} P_{j}$ and $\left.B=S^{\prime}\right)$. Now Theorem 14 implies that $\partial O$ is a 2-sphere (use $A=A_{i}$ and $B=P_{i}$ ). Since $A_{i} \subseteq T$ and $O \subseteq T$, Theorem 5 implies that $A_{i}$ and $O$ are 3-balls.

Last we show that $O$ is strongly non-shellable. We already know that $c(\Delta) \cap c(\partial O)$ is not a 2-ball for every tetrahedron $\Delta \in S^{\prime}$. We use Theorem 11 using $A=A_{i}$ and $P=P_{i}$ and see that $c(\Delta) \cap c(\partial O)$ is not a 2-ball for every tetrahedron $\Delta \in P_{i}$.

Appendix G shows that there is a strongly non-shellable 3-ball as described in Theorem 15: the strongly nonshellable 3 -ball $Z$ with 12 vertices and 25 tetrahedra in Sec. 4 of [24] ( $Z$ is the symmetric version of the 3 -ball with 10 vertices and 21 tetrahedra).

\subsection{Other shelling blocking}

After a lemma, Theorem 16 provides examples of residual 3-balls in the free-space that cannot be carved by a shelling during the surface reconstruction.

Lemma 17 If a 3-ball $O \subseteq T$ has at least two tetrahedra and $\Delta \in O$, there is a tetrahedron $\Delta^{\prime} \in O \backslash\{\Delta\}$ such that $\Delta \cap \Delta^{\prime}$ is a triangle.

Proof Assume (reductio ad absurdum) that $\partial \Delta \subseteq \partial O$. Let $O_{0}=T^{\infty} \backslash O$ and $O_{1}=O_{0} \cup\{\Delta\}$. We have
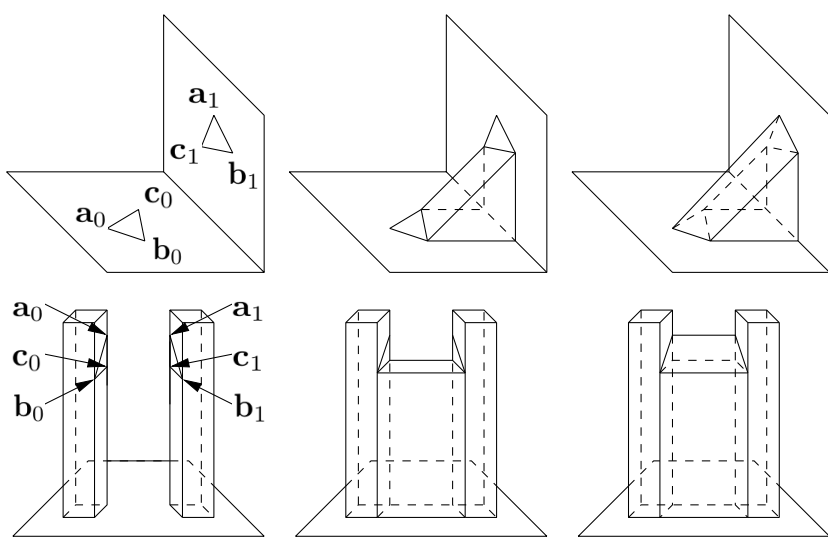

Fig. 17 Two examples for Theorem 16: $M$ includes a horizontal ground and a vertical wall (top) or includes two pillars on the ground (bottom). Left: $\partial M$ including triangles $\mathbf{a}_{0} \mathbf{b}_{0} \mathbf{c}_{0}$ and $\mathbf{a}_{1} \mathbf{b}_{1} \mathbf{c}_{1}$. Middle: $\partial M$ and $S$. Right: $\partial M$ and $S$ and $P$.

$\Delta \in T \backslash O_{0}$ and $c\left(O_{0}\right) \cap c(\Delta)=c(\partial O) \cap c(\partial \Delta)=c(\partial \Delta)$ is non-empty $2 \mathrm{D}$-pure. Thus $\left(O_{0}, O_{1}\right)$ is a shelling. Since $O_{1}=T^{\infty} \backslash(O \backslash\{\Delta\})$ and $O$ has at least two tetrahedra, $O_{1} \neq T^{\infty}$. Since $\partial O_{0}$ is a 2 -sphere and $O_{1} \neq T^{\infty}$, Corollary 2 implies that $c\left(O_{0}\right) \cap c(\Delta)=c(\partial \Delta)$ is a 2 ball (impossible).

Theorem 16 Let $M \subseteq T^{\infty}$ such that $\partial M$ is a 2-manifold. Let $S \subseteq T \backslash M$ be a 3-ball such that $c(M) \cap c(S)=$ $c\left(N \backslash\left\{\mathbf{b}_{0} \mathbf{b}_{1} \mathbf{c}_{0}, \mathbf{b}_{1} \mathbf{c}_{0} \mathbf{c}_{1}\right\}\right)$ and $N$ is an annulus (Eq. 22). Let $P=p\left(\mathbf{a}_{0} \mathbf{b}_{0} \mathbf{c}_{0}, \mathbf{a}_{1} \mathbf{b}_{1} \mathbf{c}_{1}\right)$ such that $P \subseteq T \backslash(M \cup S)$ and $c(M) \cap c(P)=c\left(\mathbf{a}_{0} \mathbf{b}_{0} \mathbf{c}_{0}, \mathbf{a}_{1} \mathbf{b}_{1} \mathbf{c}_{1}\right)$ and $c(S) \cap c(P)=$ $c\left(\mathbf{b}_{0} \mathbf{b}_{1} \mathbf{c}_{0}, \mathbf{b}_{1} \mathbf{c}_{0} \mathbf{c}_{1}\right)$. Then there is a 3-ball $S^{\prime} \subseteq S$ such that $c(M \cup P) \cap c\left(S^{\prime}\right)=c(M \cup P) \cap c(S)$ and, using notation $O=T^{\infty} \backslash\left(M \cup P \cup S^{\prime}\right)$, $\partial O$ is a 2manifold, $c(O) \cap c(\Delta)$ is not 2D-pure for every tetrahedron $\Delta \in P \cup S^{\prime}$ such that $\partial \Delta \cap \partial O \neq \emptyset$.

Two examples of $M, S$ and $P$ are shown in Fig. 17 . The first one (on the top) is inspired by the real example in Fig. 1 of [16] such that $M$ is "the matter" (set of the tetrahedra non-intersected by rays and including the ground and walls of a city), $P, S$ and $P \cup S$ are 3-balls that cannot be carved by a shelling although they are in the free-space (set of the tetrahedra intersected by rays). The second one (on the bottom) is similar and is inspired by the real example in Fig. 3.4 of [10].

Proof First we find $S^{\prime}$. Let $A=M \cup P$. Since $c(M) \cap$ $c(P)$ is two disjoint 2-balls and both $\partial M$ and $\partial P$ are 2-manifolds and $P \subset T \backslash M$, Theorem 10 implies that $\partial A$ is a 2-manifold. Furthermore, $c(A) \cap c(S)=(c(M) \cap$ $c(S)) \cup(c(P) \cap c(S))=c(N)$. Thus Corollary 4 implies that there is a 3-ball $S^{\prime} \subseteq S$ such that $c(A) \cap c\left(S^{\prime}\right)=$ $c(N)$ and $c(\Delta) \cap c\left(\partial\left(A \cup S^{\prime}\right)\right)$ is not a 2-ball for every tetrahedron $\Delta \in S^{\prime}$. 
Second we show that $\partial O$ is a 2 -manifold. Since both $\partial A$ and $\partial S^{\prime}$ are 2-manifold and $c(A) \cap c\left(S^{\prime}\right)$ is a 2manifold with boundary (the annulus $N$ ), Theorem 10 implies that $\partial\left(A \cup S^{\prime}\right)$ is a 2-manifold. Therefore $\partial O=$ $\partial\left(M \cup P \cup S^{\prime}\right)$ is a 2-manifold.

Third we show that $\Delta \in P \cup S^{\prime}$ implies that $c(\Delta) \cap$ $c(\partial O)$ is not a 2 -sphere. If $\Delta \in S^{\prime}$ and since $S^{\prime}$ is a 3 -ball with more that one tetrahedron, there is another tetrahedron $\Delta^{\prime} \in S^{\prime}$ such that $\Delta^{\prime} \cap \Delta$ is a triangle (Lemma 17). We have a similar case if $\Delta \in P$. In all cases, $\Delta$ has a triangle face $t$ between two tetrahedra in $M \cup P \cup S^{\prime}$. Since $t \notin \partial O, c(\Delta) \cap c(\partial O) \neq c(\partial \Delta)$. According to Theorem 3 and since $\Delta \notin O, c(\Delta) \cap c(\partial O)$ is not 2D-pure or is a 2-ball, but it is not a 2 -sphere.

Last we conclude. We use Theorem 11 using $A=$ $M \cup S^{\prime}$ and see that $c(\Delta) \cap c(\partial O)$ is not a 2-ball for every tetrahedron $\Delta \in P$. Now $c(\Delta) \cap c(\partial O)$ is not a 2-ball for every tetrahedron $\Delta \in P \cup S^{\prime}$. Since $c(\Delta) \cap$ $c(\partial O)$ is neither 2-ball nor 2-sphere, $c(\Delta) \cap c(\partial O)$ is not non-empty 2D-pure (Theorem 3). Since $\partial \Delta \cap \partial O \neq \emptyset$, $c(\Delta) \cap c(\partial O)$ is not $2 \mathrm{D}$ pure.

\section{Conclusion}

This article overviews shelling properties for the surface reconstruction application. In this context, a shelling is a series of tetrahedron sets $O_{0} \cdots O_{n}$ in a $3 \mathrm{D}$ Delaunay triangulation $T$, generated by a greedy algorithm that adds one tetrahedron to every $O_{i}$, such that all boundaries $\partial O_{i}$ have the same topology in almost all cases.

If $O_{0}$ includes only one tetrahedron, all $O_{i}$ are 3balls and Combinatorial Topology works study the shellability of a 3-ball $O$ : is there a shelling such that $O_{n}=$ $O$ ? Several cases are possible for greedy shelling algorithms that try to generate $O$ : always success $(O$ is extendably shellable), sometimes success ( $O$ is shellable but non-extendably shellable), and always failure ( $O$ is non-shellable). Furthermore, there are 3 invariant numbers (the $h$-numbers) for all shellings of $O$.

We also show that there is a shelling started from $O_{0}$ and ended to $O$, if $O_{0} \subset O \subseteq T$ and both $O_{0}$ and $O$ are star-shaped with respect to a same point. This generalizes a known result of shellability (if $O=T$ and $O_{0}$ has a single tetrahedron) and we use this property to qualitatively explain why the greedy algorithm does not have excessive failures and provides most of the tetrahedra enclosed by the final surface.

Last we provide a family of non-shellable 3-balls and examples of visual artifacts that a shelling alone cannot remove. Such visual artifacts occur in previous surface reconstruction results and are due to an incomplete growing of $O$ in the free-space. Actually non-shelling algorithms remove some of them, but future work is needed to remove all of them.

\section{A Proofs for prerequisites of star-shape shelling}

\section{A.1 Proof of Lemma 6}

First we show that there are points $\mathbf{x}^{\prime} \in \stackrel{\circ}{x}_{x}$ and $\mathbf{y}^{\prime} \in \mathbf{c x}^{\prime} \backslash$ $|A|$. Since $|A|$ is a closed set in $\mathbb{R}^{3}$, there is $\epsilon>0$ such that $B(\mathbf{y}, \epsilon) \cap|A|=\emptyset$. Let $\tau \in \mathbb{R}$ be such that $\mathbf{x}-\mathbf{c}=\tau(\mathbf{y}-\mathbf{c})$. We have $\tau>1$ since $\mathbf{y}$ is between $\mathbf{x}$ and $\mathbf{c}$. If $\mathbf{x} \in\left|\partial \Delta_{x}\right|$, there is a point $\mathbf{x}^{\prime}$ very close to $\mathbf{x}$ such that $\mathbf{x}^{\prime} \in \Delta_{x}$ and $\mathbf{y}^{\prime}=\mathbf{c}+\left(\mathbf{x}^{\prime}-\mathbf{c}\right) / \tau \in B(\mathbf{y}, \epsilon)$. If $\mathbf{x} \notin\left|\partial \Delta_{x}\right|$, we set $\mathbf{x}^{\prime}=\mathbf{x}$ and $\mathbf{y}^{\prime}=\mathbf{y}$.

Second we show that there are tetrahedron $\Delta_{y} \in T \backslash A$ and $\mathbf{x}^{\prime \prime} \in \dot{\Delta}_{x}$ such that $\mathbf{y}^{\prime \prime}=\mathbf{c}+\left(\mathbf{x}^{\prime \prime}-\mathbf{c}\right) / \tau \in \dot{\Delta}_{y}$. Since $|T|$ is convex and includes $\left\{\mathbf{c}, \mathbf{x}^{\prime}\right\}, \mathbf{y}^{\prime} \in|T|$. Since $\mathbf{y}^{\prime} \notin|A|$, there is a tetrahedron $\Delta_{y} \in T \backslash A$ such that $\mathbf{y}^{\prime} \in \Delta_{y}$. If $\mathbf{y}^{\prime} \in\left|\partial \Delta_{y}\right|$, there is a point $\mathbf{y}^{\prime \prime}$ very close to $\mathbf{y}^{\prime}$ such that $\mathbf{y}^{\prime \prime} \in \Delta_{y}$ and $\mathbf{x}^{\prime \prime}=\mathbf{c}+\tau\left(\mathbf{y}^{\prime \prime}-\mathbf{c}\right) \in \Delta_{x}$.

Last we find $\tilde{\mathbf{x}}$ and $\tilde{\mathbf{y}}$. Since $\Delta_{x}$ and $\Delta_{y}$ are open, we define $B_{x}^{\prime}=B\left(\mathbf{x}^{\prime \prime}, \tau \epsilon^{\prime}\right)$ and $B_{y}^{\prime}=B\left(\mathbf{y}^{\prime \prime}, \epsilon^{\prime}\right)$ such that $B_{x}^{\prime} \subset$ $\Delta_{x}$ and $B_{y}^{\prime} \subset \Delta_{y}$. Let $V$ be the union for every edge $e \in c(T)$ of the plane(s) that include(s) both $e$ and c. Since we are in a non degenerate case, $e$ and $\mathbf{c}$ are not collinear. Thus $V$ is a finite union of planes and we cannot have $B_{x}^{\prime} \subseteq V$. There is $\tilde{\mathbf{x}} \in B_{x}^{\prime} \backslash V \subseteq \Delta_{x}^{\circ}$. This implies that $\mathbf{c} \tilde{\mathbf{x}}$ does not intersect the edges in $c(T)$ and $\tilde{\mathbf{y}}=\mathbf{c}+(\tilde{\mathbf{x}}-\mathbf{c}) / \tau \in B_{y}^{\prime} \subseteq \Delta_{y}$.

\section{A.2 Proof of Lemma 7}

First we show that the set of the triangles $t_{i} \in c(T)$ intersected by (xy) are such that $\mathbf{x}_{\lambda_{i}}=(\mathbf{x y}) \cap t_{i}$ and the $\lambda_{i}$ are distinct. If $\mathbf{x}_{\lambda}$ is in a triangle $t_{\lambda} \in c(T), t_{\lambda} \cap(\mathbf{c x})$ is a line segment $\mathbf{x}_{\lambda_{0}^{\prime}} \mathbf{x}_{\lambda_{1}^{\prime}}$ (indeed the intersection of two closed convexes is a closed convex). Furthermore, $\lambda_{0}^{\prime}=\lambda_{1}^{\prime}$ (otherwise, $(\mathbf{x y})$ is in the plane of $t_{\lambda}$ and intersects an edge of $t_{\lambda}$ ) and we obtain $\mathbf{x}_{\lambda}=(\mathbf{x y}) \cap t_{\lambda}$. Let $\lambda_{i} \in \mathbb{R}$ such that $\mathbf{x}_{\lambda_{i}} \in t_{i}$. Now we show that $\lambda_{i}=\lambda_{j}$ implies $i=j$. Since (xy) does not intersect the edges of $t_{i}, \mathbf{x}_{\lambda_{i}} \in t_{i}$. Thus $t_{i}=t_{j}$ (Lemma 5), i.e. $i=j$.

Second we note that there are at least two triangles $t_{i}$, i.e. $k \geq 1$. Indeed, $\mathbf{x} \in \Delta_{x}^{\circ}$ and $\Delta_{x} \in T$ imply that (xy) intersects at least two triangles in $\partial \Delta_{x} \subset c(T)$.

Third the $\lambda_{i}$ are ordered such that $\lambda_{i}<\lambda_{i+1}$ and we show that there is a tetrahedron $\Delta_{i} \in T$ such that $t_{i} \in$ $\partial \Delta_{i}, t_{i+1} \in \partial \Delta_{i}$ and $\mathbf{x}_{\lambda} \in \Delta_{i}$ iff $\lambda \in\left[\lambda_{i}, \lambda_{i+1}\right]$. Let $\mathbf{m}_{i}=$ $\mathbf{x}_{\left(\lambda_{i}+\lambda_{i+1}\right) / 2}$. Since $|T|$ is convex, there is a tetrahedron $\Delta_{i} \in$ $T$ such that $\mathbf{m}_{i} \in \Delta_{i}$. Since $\mathbf{m}_{i}$ is not in a triangle of $c(T)$, $\mathbf{m}_{i} \in \dot{\Delta}_{i}$. Therefore $\Delta_{i} \cap(\mathbf{x y})$ is a line segment $\mathbf{x}_{\lambda_{0}^{\prime}} \mathbf{x}_{\lambda_{1}^{\prime}}$ such that $\lambda_{0}^{\prime} \neq \lambda_{1}^{\prime}$. This implies that $\mathbf{x}_{\lambda} \in \Delta_{i}$ iff $\lambda \stackrel{x_{0}}{\in}\left[\lambda_{0}^{\prime}, \lambda_{1}^{\prime}\right]$. Furthermore $\mathbf{x}_{\lambda^{\prime}} \in\left|\partial \Delta_{i}\right|$ (indeed $\mathbf{x}_{\lambda^{\prime}} \notin \check{\Delta}_{i}$ which is open). Thus there are distinct triangles $t_{j}$ and $t_{l}$ in $\partial \Delta_{i}$ such that $\mathbf{x}_{\lambda_{0}^{\prime}} \in t_{j}$ and $\mathbf{x}_{\lambda_{1}^{\prime}} \in t_{l}$ and $\lambda_{0}^{\prime}=\lambda_{j}$ and $\lambda_{1}^{\prime}=\lambda_{l}$. Since $\lambda_{j}<\lambda<\lambda_{l}$ implies $\mathbf{x}_{\lambda} \in \dot{\Delta}_{i}$, which in turn implies $\mathbf{x}_{\lambda} \notin t_{m}$ (Lemma 5), we have $j+1=l$. Since $\mathbf{m}_{i} \in \mathbf{x}_{\lambda_{j}} \mathbf{x}_{\lambda_{j+1}}$, we have $j=i$. Thus $t_{i} \in \partial \Delta_{i}, t_{i+1} \in \partial \Delta_{i}, \lambda_{0}^{\prime}=\lambda_{i}$ and $\lambda_{1}^{\prime}=\lambda_{i+1}$.

Last we assume that a tetrahedron $\Delta \in T$ meets $(\mathbf{x y}) \cap$ $\Delta \neq \emptyset$ and show that $\Delta$ is a $\Delta_{i}$. The proof is similar as in the third step above. We have $(\mathbf{x y}) \cap \Delta=\mathbf{x}_{\tilde{\lambda}_{0}} \mathbf{x}_{\tilde{\lambda}_{1}}$ such that 
$\tilde{\lambda}_{0} \leq \tilde{\lambda}_{1}$ and $\mathbf{x}_{\tilde{\lambda}_{\epsilon}}$ is in a triangle $\tilde{t}_{\epsilon} \in \partial \Delta$. Since (xy) does not intersect the $\Delta$ edges, (xy) intersects $\tilde{t_{\epsilon}}$, which in turn implies that $\tilde{\lambda}_{0} \neq \tilde{\lambda}_{1}$. Now there are distinct triangles $t_{j}$ and $t_{l}$ such that $\tilde{t_{0}}=t_{j}, \tilde{t_{1}}=t_{l}, \tilde{\lambda}_{0}=\lambda_{j}$ and $\tilde{\lambda}_{1}=\lambda_{l}$. Since $\tilde{\lambda}_{0}<\lambda<\tilde{\lambda}_{1}$ implies $\mathbf{x}_{\lambda} \in \tilde{\Delta}$, which in turn implies $\mathbf{x}_{\lambda} \notin t_{m}$ (Lemma 5), we have $l=j+1$.

\section{A.3 Proof of Theorem 7}

Let $O \subseteq T$ be c-star-shaped, a triangle $t \in \partial O$, a tetrahedron $\Delta \in O$ such that $t \in \partial \Delta$, and show that $\Delta$ and $\mathbf{c}$ are in the same $t$ half-space. If $t \in \partial T$, both $\Delta$ and $\mathbf{c}$ are in the convex set $T$ (indeed $T$ is Delaunay) and thus there are in the same half-space of $t$. If $t \notin \partial T$, there is a tetrahedron $\Delta^{\prime} \in T \backslash O$ such that $t=\Delta \cap \Delta^{\prime}$. Let $\mathbf{b}$ be the barycentre of $t$. Since $\mathbf{c}$ is not in the $t$ plane, $\stackrel{\Delta}{\Delta} \mathbf{b} \mathbf{c} \neq \emptyset$ or $\dot{\Delta}^{\prime} \cap \mathbf{b} \mathbf{c} \neq \emptyset$. Assume (reductio ad absurdum) that $\Delta^{\prime} \cap \mathbf{b c} \neq \emptyset$. Since $O$ is c-star-shaped, bc $\subseteq|O|$. Therefore there is $\Delta^{\prime \prime} \in O$ such that $\stackrel{\Delta}{ }^{\prime} \cap \Delta^{\prime \prime} \neq \emptyset$. Lemma 5 implies that $\Delta^{\prime}=\Delta^{\prime \prime} \in O$, which contradicts $\Delta^{\prime} \in T \backslash O$.

Assume that $O \subseteq T$ is not c-star-shaped and show that there is a tetrahedron $\Delta \in O$ and a triangle $t \in \partial O \cap \partial \Delta$ such that $\Delta$ and $\mathbf{c}$ are not in the same $t$ half-space. Since $O$ is not c-star-shaped, there is a point $\mathbf{x}^{\prime} \in|O| \backslash\{\mathbf{c}\}$ such that $\mathbf{c x}^{\prime}$ is not included in $|O|$. Thus there is a point $\mathbf{y}^{\prime} \in$ $\mathbf{c x}^{\prime} \backslash|O|$. Now we use Lemma 6 with $A=O$. Let $\Delta_{x} \in O$ be a tetrahedron such that $\mathbf{x}^{\prime} \in \Delta_{x}$. Thus there are tetrahedron $\Delta_{y} \in T \backslash O$ and points $\mathbf{x} \in \Delta_{x}^{\circ}$ and $\mathbf{y} \in \Delta_{y}^{\circ} \cap \mathbf{c x}$ such that $(\mathbf{c x})$ does not intersect the edges in $c(T)$. Then we obtain a tetrahedron series $\Delta_{i}$ covering $\mathbf{x y}$ as described by Lemma 7 . There are $j$ and $m$ such that $\Delta_{j}=\Delta_{x} \in O$ and $\Delta_{m}=\Delta_{y} \in$ $T \backslash O$. Since $\mathbf{x}_{0}=\mathbf{x} \in \Delta_{j}$ and $\mathbf{x}_{1}=\mathbf{y} \in \Delta_{m}, j \leq m$ and $\lambda_{m} \leq 1$. Therefore there is $l$ such that $j \leq l<m, \Delta_{l} \in O$, $\Delta_{l+1} \in T \backslash O$ and $\lambda_{l+1} \leq \lambda_{m}$. Let $\Delta=\Delta_{l}$ and the triangle $t=\Delta_{l} \cap \Delta_{l+1}$. Note that $\mathbf{x}_{\lambda} \in \Delta$ iff $\lambda \in\left[\lambda_{l}, \lambda_{l+1}\right], \mathbf{x}_{\lambda} \in t$ iff $\lambda=\lambda_{l+1}, \lambda_{l+1} \leq 1, \mathbf{c}=\mathbf{x}_{\mu}$ with $\mu>1$ ( $\mathbf{x}_{1}$ is between $\mathbf{x}_{0}$ and $\mathbf{c}$ ). Thus $\Delta$ and $\mathbf{c}$ are in different half-spaces of $t$.

\section{B Proofs for star-shape shelling}

\section{B.1 Proof of Lemma 12}

We change the coordinate basis such that $\mathbf{v}_{1}^{T}=\left(\begin{array}{lll}0 & 0 & 0\end{array}\right), \mathbf{v}_{2}^{T}=$ $\left(\begin{array}{lll}1 & 0 & 0\end{array}\right), \mathbf{v}_{3}^{T}=\left(\begin{array}{lll}0 & 1 & 0\end{array}\right), \mathbf{v}_{4}^{T}=\left(\begin{array}{lll}0 & 0 & 1\end{array}\right)$. Thus

$H_{1}^{\prime}=\left\{\left(x_{2}, x_{3}, x_{4}\right)^{T} \in \mathbb{R}^{3}, x_{2}+x_{3}+x_{4} \geq 1\right\}$

$H_{2}^{\prime}=\left\{\left(x_{2}, x_{3}, x_{4}\right)^{T} \in \mathbb{R}^{3}, x_{2} \leq 0\right\}$

$H_{3}^{\prime}=\left\{\left(x_{2}, x_{3}, x_{4}\right)^{T} \in \mathbb{R}^{3}, x_{3} \leq 0\right\}$

$H_{4}^{\prime}=\left\{\left(x_{2}, x_{3}, x_{4}\right)^{T} \in \mathbb{R}^{3}, x_{4} \leq 0\right\}$.

Now we see that $\left(x_{2}, x_{3}, x_{4}\right) \in H_{1}^{\prime} \cap H_{2}^{\prime} \cap H_{3}^{\prime} \cap H_{4}^{\prime}$ is impossible.

Assume that $\mathbf{c} \in H_{1}^{\prime} \cap H_{2} \cap H_{3} \cap H_{4}$. We have $\mathbf{v}_{1} \in H_{2} \cap$ $H_{3} \cap H_{4}$ (indeed $\Delta=H_{1} \cap H_{2} \cap H_{3} \cap H_{4}$ ). Since $H_{2} \cap H_{3} \cap H_{4}$ is convex and includes both $\mathbf{c}$ and $\mathbf{v}_{1}, \mathbf{c v}_{1} \subset H_{2} \cap H_{3} \cap H_{4}$. Let plane $\pi_{1}=H_{1} \cap H_{1}^{\prime}$. Thus $\mathbf{c v}_{1} \cap \pi_{1} \subseteq H_{1} \cap H_{2} \cap H_{3} \cap H_{4} \cap H_{1}^{\prime}=$ $\Delta \cap \pi_{1}=t_{1}$. We obtain $\mathbf{c v}_{1} \cap \pi_{1} \subseteq \mathbf{c v}_{1} \cap t_{1}$. Since $\mathbf{c}$ and $\mathbf{v}_{1}$ are in different $t_{1}$ half-spaces, $\mathbf{c v}_{1} \cap \pi_{1} \neq \emptyset$ and we obtain $\mathbf{c v}_{1} \cap t_{1} \neq \emptyset$.

Assume that $\mathbf{c} \in H_{1}^{\prime} \cap H_{2}^{\prime} \cap H_{3} \cap H_{4}$. The tetrahedron $\Delta=\mathbf{v}_{1} \mathbf{v}_{2} \mathbf{v}_{3} \mathbf{v}_{4}$ is split in two tetrahedra $\Delta_{1}=\mathbf{v}_{1} \tilde{\mathbf{v}}_{2} \mathbf{v}_{3} \mathbf{v}_{4}$ and $\Delta_{2}=\tilde{\mathbf{v}}_{1} \mathbf{v}_{2} \mathbf{v}_{3} \mathbf{v}_{4}$ using $\tilde{\mathbf{v}}_{2}=\tilde{\mathbf{v}}_{1}=\mathbf{v}=\left(\mathbf{v}_{1}+\mathbf{v}_{2}\right) / 2$. Let $\tilde{H}_{1}$ and $\tilde{H}_{2}$ be the half-spaces of the triangle $\mathbf{v} \mathbf{v}_{3} \mathbf{v}_{4}$ such
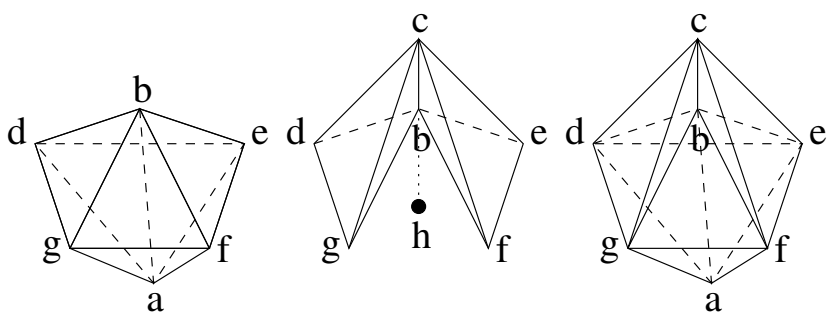

Fig. 18 A non-shellable star-shape $O$ with respect to $\mathbf{h}$ such that $\mathbf{h} \notin|\partial O|$ (right): $O$ is the union of $\mathbf{a b} \times \mathbf{d}$-e-f-g-d (left) and $\{$ bcef, bcgd $\}$ (middle).

that $\mathbf{v}_{1} \in \tilde{H}_{1}$ and $\mathbf{v}_{2} \in \tilde{H}_{2}$. Note that $\Delta_{2}$ is like $\Delta$ except that the vertex $\mathbf{v}_{1}$ is replaced by $\tilde{\mathbf{v}}_{1}$ and the half-space $H_{2}$ is replaced by $\tilde{H}_{2}: \Delta_{2}=H_{1} \cap \tilde{H}_{2} \cap H_{3} \cap H_{4}$ ( $t_{1}$ does not change, the half-spaces $H_{3}$ and $H_{4}$ does not change since their boundary planes include $\tilde{\mathbf{v}}_{1}, \mathbf{v}_{1}$ and $\mathbf{v}_{2}$ that are collinear). Assume $\mathbf{c} \in \tilde{H}_{2}$ (the other case $\mathbf{c} \in \tilde{H}_{1}$ is similar). We have $\mathbf{c} \in H_{1}^{\prime} \cap \tilde{H}_{2} \cap H_{3} \cap H_{4}$ (indeed, c $\in H_{1}^{\prime} \cap H_{3} \cap H_{4}$ and c $\in \tilde{H}_{2}$ ). According to the second assertion of the lemma applied to $\Delta_{2}, \mathbf{c} \tilde{\mathbf{v}}_{1} \cap t_{1} \neq \emptyset$. Thus $\mathbf{c v} \cap\left(t_{1} \cup t_{2}\right) \neq \emptyset$.

\section{Non-shellable star-shapes in degenerate cases}

Here we show that there are star-shapes $O$ that are non shellable in degenerate cases. More precisely, we find $\mathbf{h}$-starshapes $O$ such that $\partial O$ has a singular vertex and $\mathbf{h}$ is in a plane of a triangle in $\partial O$. Since $\partial O$ is a 2-manifold if $O$ is shellable (Theorem 4), we see that such an $O$ is non-shellable.

For example, $O=\{$ habc, $\mathbf{h d e f}\}$ is a $\mathbf{h}$-star-shape such that $\partial O$ has a singular vertex $\mathbf{h}$. Fig. 18 shows another example $O$ that is star-shaped with respect to a point $\mathbf{h} \notin|\partial O|$ :

$O=S_{1} \cup\{$ bcef, bcgd $\}$ where $S_{1}=\mathbf{a b} \times \mathbf{d}$-e-f-g-d.

Note that $\mathbf{h}$ is in the plane of the triangle bcg $\in \partial O$ and $\mathbf{b}$ is a singular vertex of $\partial O$. Now we show that $O$ can be star-shaped.

Property 2 Let $S_{2}=\mathbf{b c} \times \mathbf{d}-\mathbf{e}-\mathbf{f}-\mathbf{g}-\mathbf{d}$ and $\mathbf{h} \in\left|S_{1}\right| \cap(\mathbf{b c})$. If both $S_{1}$ and $S_{1} \cup S_{2}$ are convex, $O$ is h-star-shaped.

Proof Let $\mathbf{x} \in|O|$ and show that $\mathbf{x h} \subseteq|O|$. We have $\mathbf{x} \in\left|S_{1}\right|$ or $\mathbf{x} \in \mathbf{b c e f}$ (or similarly $\mathbf{x} \in \mathbf{b c g d}$ ). Since $S_{1}$ is convex and $\mathbf{h} \in\left|S_{1}\right|, \mathbf{x} \in\left|S_{1}\right|$ implies $\mathbf{x h} \subseteq\left|S_{1}\right| \subseteq|O|$.

Now we assume $\mathbf{x} \in \mathbf{b c e f}$ and show $\mathbf{x h} \cap \mathbf{b c d e}=\mathbf{x h} \cap$ bce. Since $\mathbf{h} \in(\mathbf{b c})$ and $\mathbf{x} \in \mathbf{b c e f}, \mathbf{h x}$ and bcef are in the same half-space of bce. Since bcde is in the other half-space of bce, $\mathbf{h x} \cap \mathbf{b c d e}$ is included in the plane of bce. We obtain $\mathbf{x h} \cap \mathbf{b c d e}=\mathbf{x h} \cap \mathbf{b c e}$ and similarly $\mathbf{x h} \cap \mathbf{b c f g}=\mathbf{x h} \cap \mathbf{b c f}$.

Since $S_{1} \cup S_{2}$ is convex and $\mathbf{h} \in\left|S_{1} \cup S_{2}\right|, \mathbf{x h} \subseteq\left|S_{1} \cup S_{2}\right|=$ $\mid O \cup\{$ bcde, $\mathbf{b c f g}\} \mid$. Thus

$\mathbf{x h}=\mathbf{x h} \cap|O \cup\{\mathbf{b c d e}, \mathbf{b c f g}\}|=\mathbf{x h} \cap(|O| \cup \mathbf{b c e} \cup \mathbf{b c f})(31)$

Since bce $\subseteq|O|$ and bcf $\subseteq|O|$, we obtain $\mathbf{x h} \subseteq|O|$.

\section{Proof of Theorem 10}

Lemma 18 Assume that $A \subseteq T^{\infty}$ and $B \subseteq T \backslash A$. Then $c(A) \cap c(B)$ is a simplicial complex in $\mathbb{R}^{3}$. Furthermore, $c(A) \cap$ $c(B)=c(\partial A) \cap c(\partial B)$. 
Proof This is Lemma 1 in [14] by replacing the set $B$ by a single tetrahedron. The proof is very similar as the proof in Appendix A.1 of [14].

We show that every vertex $\mathbf{v} \in c(\partial(A \cup B))$ meets $(\partial(A \cup$ $B))_{\mathbf{v}}=\mathbf{v} \times \mathbf{x}_{*}$ where $\mathbf{x}_{*}$ is a cycle. Thanks to Lemma 15 $\partial(A \cup B)=(\partial A \backslash \partial B) \cup(\partial B \backslash \partial A)$. Thus $\mathbf{v} \in c(\partial A)$ or $\mathbf{v} \in c(\partial B)$. We also have

$(\partial(A \cup B))_{\mathbf{v}}=\left((\partial A)_{\mathbf{v}} \backslash(\partial B)_{\mathbf{v}}\right) \cup\left((\partial B)_{\mathbf{v}} \backslash(\partial A)_{\mathbf{v}}\right)$.

There are three cases. If $\mathbf{v} \in c(\partial A) \backslash c(\partial B),(\partial B)_{\mathbf{v}}=\emptyset$. Thanks to Eq. 32, $(\partial(A \cup B))_{\mathbf{v}}=(\partial A)_{\mathbf{v}}$. Since $\partial A$ is a 2 manifold, there is a cycle $\mathbf{x}_{*}$ such that $(\partial(A \cup B))_{\mathbf{v}}=(\partial A)_{\mathbf{v}}=$ $\mathbf{v} \times \mathbf{x}_{*}$. The proof is the same if $\mathbf{v} \in c(\partial B) \backslash c(\partial A)$.

Now we consider the last case $\mathbf{v} \in c(\partial A) \cap c(\partial B)$. Since $\partial A$ is a 2-manifold,

$\exists \mathbf{a}_{i} \in c(\partial A),(\partial A)_{\mathbf{v}}=\mathbf{v} \times \mathbf{a}_{*}, \mathbf{a}_{*}=\mathbf{a}_{1}-\cdots \mathbf{a}_{m}-\mathbf{a}_{1}$,

and $\mathbf{a}_{*}$ is a cycle. Since $\partial B$ is a 2 -manifold,

$\exists \mathbf{b}_{i} \in c(\partial B),(\partial B)_{\mathbf{v}}=\mathbf{v} \times \mathbf{b}_{*}, \mathbf{b}_{*}=\mathbf{b}_{1}-\cdots \mathbf{b}_{n}-\mathbf{b}_{1}$,

and $\mathbf{b}_{*}$ is a cycle. Since $c(\partial A) \cap c(\partial B)=c(A) \cap c(B)$ (Lemma 18) and $c(A) \cap c(B)$ is a 2-manifold with boundary, there are $\mathbf{c}_{i} \in c(\partial A) \cap c(\partial B)$ such that

$(\partial A \cap \partial B)_{\mathbf{v}}=\mathbf{v} \times \mathbf{c}_{*}, \mathbf{c}_{*}=\mathbf{c}_{1} \cdots \mathbf{c}_{k}$,

and there are two cases: $\mathbf{c}_{*}$ is a cycle and $k \geq 3$, or all $\mathbf{c}_{i}$ are distinct and $k \geq 2$. Since $(\partial A \cap \partial B)_{\mathbf{v}} \subseteq(\partial A)_{\mathbf{v}}$ and $(\partial A \cap$ $\partial B)_{\mathbf{v}} \subseteq(\partial B)_{\mathbf{v}}$, we have $\mathbf{c}_{*} \subseteq \mathbf{a}_{*}$ and $\mathbf{c}_{*} \subseteq \mathbf{b}_{*}$. Assume (reductio ad absurdum) that $\mathbf{c}_{*}$ is a cycle. Since $\mathbf{c}_{*}$ is included in $\mathbf{a}_{*}$ and $\mathbf{b}_{*}$ that are also cycles, $\mathbf{a}_{*}=\mathbf{c}_{*}=\mathbf{b}_{*}$. This implies $(\partial A)_{\mathbf{v}}=(\partial B)_{\mathbf{v}}$. Thanks to Eq. $32,(\partial(A \cup B))_{\mathbf{v}}=\emptyset$. This contradicts $\mathbf{v} \in c(\partial(A \cup B))$.

Now the $\mathbf{c}_{i}$ are distinct and $k \geq 2$. Since the $\mathbf{c}_{i}$ are distinct and $\mathbf{c}_{*} \subseteq \mathbf{a}_{*}, k \leq m$. We shift the $\mathbf{a}_{i}$ such that $\mathbf{a}_{i}=\mathbf{c}_{i}$ if $1 \leq i \leq \bar{k}$. Similarly $k \leq n$ and $\mathbf{b}_{i}=\mathbf{c}_{i}$ if $1 \leq i \leq k$. Thanks to Eq. $32,(\partial(A \cup B))_{\mathbf{v}}=\mathbf{v} \times \mathbf{x}_{*}$ where

$\mathbf{x}_{*}=\mathbf{a}_{k^{-}} \cdots \mathbf{a}_{m}-\mathbf{a}_{1} \cup \mathbf{b}_{k^{-}} \cdots \mathbf{b}_{n}-\mathbf{b}_{1}$.

Since $\mathbf{a}_{1}=\mathbf{b}_{1}=\mathbf{c}_{1}$ and $\mathbf{a}_{k}=\mathbf{b}_{k}=\mathbf{c}_{k}$,

$\mathbf{x}_{*}=\mathbf{c}_{k}-\mathbf{a}_{k+1}-\mathbf{a}_{k+2^{-}} \cdots \mathbf{a}_{m}-\mathbf{c}_{1}-\mathbf{b}_{n}-\mathbf{b}_{n-1^{-}} \cdots \mathbf{b}_{k+1}-\mathbf{c}_{k}$.

Then we show that $c\left(\mathbf{x}_{*}\right)$ has at least 3 distinct vertices. It has $\mathbf{c}_{k}$ and $\mathbf{c}_{1}$. Furthermore, $\mathbf{c}_{1} \neq \mathbf{c}_{k}$ since $k \geq 2$. Assume (reduction ad absurdum) that it has no other vertex. Thus $m=k=n$. This implies $(\partial A)_{\mathbf{v}}=(\partial B)_{\mathbf{v}}$, which is impossible (see above).

Last we show that the vertices in the vertex sequence $\mathbf{x}_{*}$ are distinct. We have $\mathbf{c}_{1} \neq \mathbf{c}_{k}$ since $k \geq 2$. We also have $\mathbf{c}_{1} \neq \mathbf{a}_{i}$ if $i>k$, since $\mathbf{c}_{1}=\mathbf{a}_{1}$ and the $\mathbf{a}_{i}$ are distinct (indeed, $\mathbf{a}_{*}$ is a cycle). Similarly, $\mathbf{c}_{1} \neq \mathbf{b}_{i}$ if $i>k, \mathbf{c}_{k} \neq \mathbf{a}_{i}$ and $\mathbf{c}_{k} \neq \mathbf{b}_{i}$ if $i>k$. Assume (reductio ad absurdum) that $\mathbf{a}_{i}=\mathbf{b}_{j}$ if $i>k$ and $j>k$. Thus $\mathbf{v a}_{i} \in c(\partial A) \cap c(\partial B)$. Since $c(\partial A) \cap c(\partial B)$ is $2 \mathrm{D}$ pure $^{2}$ (indeed, $c(\partial A) \cap c(\partial B)$ is a 2 -manifold with boundary), there is a triangle $t \in(\partial A \cap \partial B)_{\mathbf{v}}$ such that $\mathbf{a}_{i} \in t$. This implies that $\mathbf{a}_{i}=\mathbf{a}_{j}$ with $i>k$ and $j \leq k$ (impossible).

2 Addendum: use Lemma 24 in Appendix H.

\section{E Proof of Theorem 12}

First Theorem 17 details the splitting of a 2-sphere in $c(T)$ by a cycle. Then we show Theorem 12 (splitting of $\partial S$ by the annulus $N$ ) by applying Theorem 17 to every cycle in $\partial N$.

Theorem 17 Let $L \subseteq T$ be a 3-ball and $\mathbf{b}_{*} \in c(\partial L)$ be a cycle. There are $B_{i}$ such that $B_{1} \cup B_{2}=\partial L, \partial B_{1}=\partial B_{2}=$ $\mathbf{b}_{*}, c\left(B_{1}\right) \cap c\left(B_{2}\right)=c\left(\mathbf{b}_{*}\right)$, every $B_{i}$ is strongly connected.

Intuitively, the closed curve $\mathbf{b}_{*}$ splits the 2 -sphere $\partial L$ in two disjoint and connected sets (2-balls) of triangles $B_{1}$ and $B_{2}$ that have the common boundary $\mathbf{b}_{*}$. This is a discrete version of the Jordan Theorem for a 2-sphere encoded by a simplicial complex.

Proof Since the 3-ball $L \subseteq T, \partial L$ is a 2 -sphere (Theorem 5). Let $\mathbf{m} \in|\partial L|$ that is neither a vertex nor in an edge of $c(\partial L)$. Let $\varphi$ be a homeomorphism such that $\varphi(|\partial L| \backslash\{\mathbf{m}\})=\mathbb{R}^{2}$.

Let $G=(V, E)$ be the graph of the vertices and edges in $c(\partial L)$. Let $G^{*}=\left(V^{*}, E^{*}\right)$ be the adjacency graph of the triangles in $\partial L$, i.e. $V^{*}=\{v(t), t \in \partial L\}$ and $E^{*}=$ $\left\{e\left(t_{1}, t_{2}\right), t_{i} \in \partial L, t_{1} \cap t_{2}\right.$ is an edge $\}$. Both $G$ and $G^{*}$ have drawings (mappings) in $\mathbb{R}^{2}$ by $\varphi$. The drawing of $G$ is $(\varphi(V), \varphi(E))$. The drawing of $v(t) \in V^{*}$ is the drawing of the barycentre of $t \in \partial L$. The drawing of $e\left(t_{1}, t_{2}\right) \in E^{*}$ is the drawing of a polygonal curve linking the barycentres of adjacent triangles $t_{1}$ and $t_{2}$ (the polygonal curve is included in $t_{1} \cup t_{2}$ and intersects the edge $\left.t_{1} \cap t_{2}\right)$. Let $F=\mathbf{b}_{*}$ and $F^{*}$ be the dual edges of $F$, i.e. $F^{*}=\left\{e\left(t_{1}, t_{2}\right) \in E^{*}, t_{1} \cap t_{2} \in F\right\}$. Thanks to Proposition 4.6.1 in [6] (the plane duality theorem) and since $G$ is connected and the drawings of $G$ and $G^{*}$ are dual and $F$ is a cycle, $F^{*}$ is a minimal cut of $G^{*}$.

Word "cut" means that the graph $\left(V^{*}, E^{*} \backslash F^{*}\right)$ is disconnected. Word "minimal" means that $\left(V^{*}, E^{*} \backslash F^{*}\right)$ becomes connected if we remove any edge from $F^{*}$. If we don't remove an edge from $F^{*},\left(V^{*}, E^{*} \backslash F^{*}\right)$ has exactly two connected components $\left\{v(t), t \in B_{1}\right\}$ and $\left\{v(t), t \in B_{2}\right\}: B_{1} \cup B_{2}=\partial L$, $B_{1} \cap B_{2}=\emptyset$, and every $B_{i}$ is non-empty strongly connected using the edges in $E^{*} \backslash F^{*}$.

Let edge $e \in \partial B_{i}$ and show $e \in \mathbf{b}_{*}$. Since $\partial L$ is a 2manifold and the $B_{i}$ partition $\partial L$, there are triangles $t_{i} \in$ $B_{i}$ such that $e=t_{1} \cap t_{2}$. If $e\left(t_{1}, t_{2}\right) \in E^{*} \backslash F^{*}, v\left(t_{1}\right)$ and $v\left(t_{2}\right)$ are in the same connected component of $\left(V^{*}, E^{*} \backslash F^{*}\right)$ (impossible). Thus $e\left(t_{1}, t_{2}\right) \in F^{*}$ and $e \in F=\mathbf{b}_{*}$.

Let edge $e \in \mathbf{b}_{*}$ and show $e \in \partial B_{i}$. We have distinct triangles $t_{i} \in \partial L$ such that $e=t_{1} \cap t_{2}$. Since $F=\mathbf{b}_{*}$, $e\left(t_{1}, t_{2}\right) \in F^{*}$. If $t_{1} \in B_{1}$ and $t_{2} \in B_{1}$ (reductio ad absurdum), we remove the edge $e\left(t_{1}, t_{2}\right)$ from $F^{*}$ and add it to $E^{*} \backslash F^{*}$ without connecting $B_{1}$ and $B_{2}$, i.e. $F$ is not a minimal cut (impossible). Thus $t_{1} \in B_{1}$ and $t_{2} \in B_{2}$; we obtain $e \in \partial B_{i}$.

Since $\partial B_{i}=\mathbf{b}_{*}, c\left(\mathbf{b}_{*}\right) \subseteq c\left(B_{1}\right) \cap c\left(B_{2}\right)$. Now we show $c\left(B_{1}\right) \cap c\left(B_{2}\right) \subseteq c\left(\mathbf{b}_{*}\right)$. Since $B_{1} \cap B_{2}=\emptyset$, there are not triangles in $c\left(\overline{B_{1}}\right) \cap c\left(B_{2}\right)$. Let edge $e \in c\left(B_{1}\right) \cap c\left(B_{2}\right)$; e is included in a triangle in $B_{1}$ and another in $B_{2}$ and thus $e \in \mathbf{b}_{*}$. Let vertex $\mathbf{v} \in c\left(B_{1}\right) \cap c\left(B_{2}\right)$. Since $\partial L$ is a 2 -manifold, there is a triangle series $t_{i} \in(\partial L)_{\mathbf{v}}$ such that every $t_{i} \cap t_{i+1}$ is an edge. There is a $t_{j} \in B_{1}$ and $t_{k} \in B_{2}$. Therefore there is $i$ such that $t_{i} \in B_{1}$ and $t_{i+1} \in B_{2}$. We see that the edge $e=t_{i} \cap t_{i+1}$ meets $e \in c\left(B_{1}\right) \cap c\left(B_{2}\right)$. Since $e \in \mathbf{b}_{*}$ (see above), $\mathbf{v} \in c\left(\mathbf{b}_{*}\right)$.

Now we do the proof of Theorem 12 .

First we introduce notations by applying Theorem 17 to cycles $\mathbf{b}_{*}$ and $\mathbf{c}_{*}$. There are $B_{i}$ such that $B_{1} \cup B_{2}=\partial L$, $\partial B_{1}=\partial B_{2}=\mathbf{b}_{*}, c\left(B_{1}\right) \cap c\left(B_{2}\right)=c\left(\mathbf{b}_{*}\right)$ and every $B_{i}$ is strongly connected. There are $C_{i}$ such that $C_{1} \cup C_{2}=\partial L$, 
$\partial C_{1}=\partial C_{2}=\mathbf{c}_{*}, c\left(C_{1}\right) \cap c\left(C_{2}\right)=c\left(\mathbf{c}_{*}\right)$ and every $C_{i}$ is strongly connected.

Second we show that $N \subseteq B_{2}$ (similarly, $N \subseteq C_{2}$ ). Assume (reductio ad absurdum) that there are triangles $t_{1} \in$ $N \cap B_{1}$ and $t_{2} \in N \cap B_{2}$. Since $N$ is strongly connected and $N \subseteq B_{1} \cup B_{2}$, we can choose $t_{1}$ and $t_{2}$ such that $t_{1} \cap t_{2}$ is an edge. Therefore $t_{1} \cap t_{2} \in \partial B_{i}=\mathbf{b}_{*}$. However, an edge between two distinct triangles in $N$ is $\mathbf{b}_{i} \mathbf{c}_{i}$ or $\mathbf{b}_{i+1} \mathbf{c}_{i}$ or $\mathbf{c}_{i+1} \mathbf{b}_{i}$ (contradiction). We obtain $N \subseteq B_{2}$.

Third we show that $c(N) \cap c\left(B_{1}\right)=c\left(\mathbf{b}_{*}\right)$ (similarly, $\left.c(N) \cap c\left(C_{1}\right)=c\left(\mathbf{c}_{*}\right)\right)$. Since $c\left(\mathbf{b}_{*}\right) \subseteq c\left(B_{1}\right)$ and $c\left(\mathbf{b}_{*}\right) \subseteq$ $c(N) \subseteq c\left(B_{2}\right)$ and $c\left(B_{1}\right) \cap c\left(B_{2}\right)=c\left(\overline{\mathbf{b}}_{*}\right)$, we have

$c\left(\mathbf{b}_{*}\right) \subseteq c(N) \cap c\left(B_{1}\right) \subseteq c\left(B_{1}\right) \cap c\left(B_{2}\right) \subseteq c\left(\mathbf{b}_{*}\right)$.

Thus $c(N) \cap c\left(B_{1}\right)=c\left(\mathbf{b}_{*}\right)$.

Fourth we show that $B_{1} \cap C_{1}=\emptyset$. Since $\partial B_{1}=\mathbf{b}_{*}$, there is a triangle $\tilde{t}=\mathbf{d b}_{0} \mathbf{b}_{1} \in B_{1}$. Assume (reductio ad absurdum) that $\tilde{t} \in C_{1}$. Thus $\mathbf{b}_{0} \in c\left(C_{1}\right)$. Since $\mathbf{b}_{0} \in c(N)$, we have $\mathbf{b}_{0} \in c\left(C_{1}\right) \cap c(N)=c\left(\mathbf{c}_{*}\right)$, i.e. $\mathbf{b}_{0}$ is equal to a $\mathbf{c}_{j}$ (impossible). We see that every triangle $\tilde{t}=\mathbf{d b}_{0} \mathbf{b}_{1} \in B_{1} \backslash C_{1}$. Assume (reductio ad absurdum) that there is a triangle $t \in$ $C_{1} \cap B_{1}$. Since $B_{1}$ is strongly connected and $\tilde{t} \in B_{1} \backslash C_{1}$ and $t \in B_{1} \cap C_{1}$, we can find triangles $t^{\prime} \in B_{1} \backslash C_{1}$ and $t^{\prime \prime} \in B_{1} \cap C_{1}$ such that $t^{\prime} \cap t^{\prime \prime}$ is an edge. Therefore $t^{\prime} \cap t^{\prime \prime} \in$ $\partial C_{1}=\mathbf{c}_{*} \subseteq \partial N$. This implies that $t^{\prime} \in N$ or $t^{\prime \prime} \in N$, which is impossible since $B_{1} \cap N \subseteq B_{1} \cap B_{2}=\emptyset$.

Fifth we show that $N \cup C_{1}=B_{2}$. Since $B_{1} \cap C_{1}=\emptyset$ and $C_{1} \subseteq B_{1} \cup B_{2}, C_{1} \subseteq B_{2}$. Thus $N \cup C_{1} \subseteq B_{2}$ and we only need to show that $t^{\prime \prime \prime} \in B_{2}$ implies $t^{\prime \prime \prime} \in \bar{N} \cup C_{1}$. Since $B_{2}$ is strongly connected and $\emptyset \neq N \cup C_{1} \subseteq B_{2}$, there is a triangle series $t_{i} \in B_{2}$ such that $t_{0} \in N \cup C_{1}$ and $t_{n}=t^{\prime \prime \prime}$ and every $t_{i} \cap t_{i+1}$ is an edge that is not in $\partial B_{2}$. Since $N \cap C_{1}=\emptyset$,

$$
\begin{aligned}
\partial\left(N \cup C_{1}\right) & =\left(\partial N \backslash \partial C_{1}\right) \cup\left(\partial C_{1} \backslash \partial N\right) \\
& =\left(\left(\mathbf{b}_{*} \cup \mathbf{c}_{*}\right) \backslash \mathbf{c}_{*}\right) \cup\left(\mathbf{c}_{*} \backslash\left(\mathbf{b}_{*} \cup \mathbf{c}_{*}\right)\right) \\
& =\mathbf{b}_{*}=\partial B_{2} .
\end{aligned}
$$

Therefore every $t_{i} \cap t_{i+1}$ is not in $\partial\left(N \cup C_{1}\right)$. Now we have $t_{i+1} \in N \cup C_{1}$ if $t_{i} \in N \cup C_{1}$. Since $t_{0} \in N \cup C_{1}$, we obtain $t^{\prime \prime \prime}=t_{n} \in N \cup C_{1}$.

Last we show $c\left(B_{1}\right) \cap c\left(C_{1}\right)=\emptyset$ and conclude using $B=$ $B_{1}$ and $C=C_{1}$. Since $C_{1} \subseteq B_{2}$ (see above), $c\left(B_{1}\right) \cap c\left(C_{1}\right) \subseteq$ $c\left(B_{1}\right) \cap c\left(B_{2}\right)=c\left(\mathbf{b}_{*}\right)$. Furthermore $B_{1} \subseteq C_{2}$ (indeed, $B_{1} \cap$ $C_{1}=\emptyset$ and $B_{1} \subseteq C_{1} \cup C_{2}$ imply $\left.B_{1} \subseteq C_{2}\right)$. Thus $c\left(B_{1}\right) \cap$ $c\left(C_{1}\right) \subseteq c\left(C_{2}\right) \cap c\left(C_{1}\right)=c\left(\mathbf{c}_{*}\right)$. We obtain $c\left(B_{1}\right) \cap c\left(C_{1}\right) \subseteq$ $c\left(\mathbf{b}_{*}\right) \cap c\left(\mathbf{c}_{*}\right)=\emptyset$.

\section{F Proof of Theorem 14}

We show that $\partial(A \cup B)$ is a connected 2-manifold that has the same genus as $\partial A$ (therefore they are homeomorphic). The principle of the proof is the following. Theorem 10 implies that $\partial(A \cup B)$ is a 2 -manifold. The set $D=\partial A \cap \partial B$ is a 2ball $^{3}$. Then we show that $\partial(A \cup B)$ is connected by studying the connectivity of $\partial A \backslash D$ and $\partial B \backslash D$ and since their closures include the 1-sphere $\partial D$. Last we use properties of Euler's characteristic $\chi(X)$ of $X \subseteq c(T)$ to show that both $\partial(A \cup B)$ and $\partial A$ have the same genus. We remind that $\chi(X)$ is the number of the vertices plus number of the triangles minus the number of the edges in $X$, thus it is closely related to the genus of $X$ (Sec. 2.6).

We start by the two following lemmas, which are useful to show that $\partial A \backslash D$ and $\partial B \backslash D$ are connected.

\footnotetext{
3 Addendum: more details in Appendix H.
}

Lemma 19 Let $M \subseteq c(T)$ be a 2-manifold ${ }^{4}$. Let $D \subset M$. Then $\partial(M \backslash D)=\partial D$ and $c(M \backslash D) \cap c(D)=c(\partial D)$.

Proof First we show $\partial D=\partial(M \backslash D)$. Let $e \in c(M)$ be an edge. Since $M$ is a 2-manifold, there are exactly two triangles $t_{1}$ and $t_{2}$ in $M$ that includes $e$ and $e=t_{1} \cap t_{2}$. Since $D \subseteq M$, $e \in \partial D$ iff $t_{1} \in D$ and $t_{2} \in M \backslash D$ iff $e \in \partial(M \backslash D)$.

If a simplex $\sigma \in c(\partial D), \sigma \in c(D)$ and $\sigma \in c(\partial(M \backslash D)) \subseteq$ $c(M \backslash D)$. Therefore $\sigma \in c(M \backslash D) \cap c(D)$. Conversely, let $\sigma \in$ $c(M \backslash D) \cap c(D)$ and show $\sigma \in c(\partial D)$. This means that there are triangles $t_{1} \in D$ and $t_{2} \in M \backslash D$ such that $\sigma \subseteq t_{1} \cap t_{2}$. Assume that $\sigma$ is an edge (case 1). Thus $\sigma=t_{1} \cap t_{2}$. Since $D \subseteq M$ and $M$ is a 2-manifold, the only triangles in $M$ that includes $\sigma$ are $t_{1}$ and $t_{2}$. Therefore there is only one triangle in $D$ that includes $\sigma$ and we obtain $\sigma \in c(\partial D)$. Assume that $\sigma$ is a vertex (case 2). Since $M$ is a 2 -manifold, $M_{\mathbf{v}}$ is a triangle series $t_{i}^{\prime} \in M$ such that every $t_{i}^{\prime} \cap t_{i+1}^{\prime}$ is an edge including $\mathbf{v}^{5}$. There is $j$ and $k$ such that $t_{j}^{\prime}=t_{1} \in D$ and $t_{k}^{\prime}=t_{2} \in M \backslash D$. Thus we can find $i$ such that $t_{i}^{\prime} \in D$ and $t_{i+1}^{\prime} \in M \backslash D$ (or vice versa). Now vertex $\sigma$ is a face of the edge $e=t_{i}^{\prime} \cap t_{i+1}^{\prime}$ such that $e \in c(M \backslash D) \cap c(D)$ and we just showed (case 1) that $e \in c(\partial D)$. Therefore $\sigma \in c(\partial D)$.

Lemma 20 Assume that $M \subseteq c\left(T^{\infty}\right)$ is a connected 2manifold $^{6}$. If a 2-ball $D \subseteq M, \bar{M} \backslash D$ is connected.

Proof Let vertices $\mathbf{v}$ and $\mathbf{v}^{\prime}$ in $c(M \backslash D)$. Since they are in $c(M)$ and $M$ is connected, there is a path $\mathbf{v}_{0}-\mathbf{v}_{1}-\cdots \mathbf{v}_{n} \subseteq$ $c(M)$ such that $\mathbf{v}_{0}=\mathbf{v}$ and $\mathbf{v}_{n}=\mathbf{v}^{\prime}$. If this path has an edge which is not in $c(M \backslash D)$, we modify it to obtain another path such that all its edges are in $c(M \backslash D)$ (thus $M \backslash D$ is connected).

Let $j$ be the smallest index $i$ such that $\mathbf{v}_{i} \mathbf{v}_{i+1} \notin c(M \backslash D)$. We show that $\mathbf{v}_{j} \in c(\partial D)$. Since $\mathbf{v}_{j} \mathbf{v}_{j+1} \in c(M)=c(M \backslash$ $D) \cup c(D), \mathbf{v}_{j} \mathbf{v}_{j+1} \in c(D)$. If $j=0$, we have $\mathbf{v}_{j} \in c(M \backslash D) \cap$ $c(D)=c(\partial D)$ (Lemma 19). If $j>0, \mathbf{v}_{j-1} \mathbf{v}_{j} \in c(M \backslash D)$ and we have $\mathbf{v}_{j} \in c(M \backslash D) \cap c(D)=c(\partial D)$.

Let $k$ be the greatest index $i$ such that $\mathbf{v}_{i} \mathbf{v}_{i+1} \notin c(M \backslash D)$. Similarly, $\mathbf{v}_{k+1} \in c(\partial D)$.

Since $D$ is a 2-ball, $\partial D$ is a cycle of edges $\mathbf{d}_{i} \mathbf{d}_{i+1}$. There are integers $l$ and $m$ such that the path $\mathbf{d}_{l}-\mathbf{d}_{l+1^{-}} \cdots \mathbf{d}_{m} \subset \partial D$ meets $\mathbf{d}_{l}=\mathbf{v}_{j}$ and $\mathbf{d}_{m}=\mathbf{v}_{k+1}$. Thanks to Lemma 19, every edge $\mathbf{d}_{i} \mathbf{d}_{i+1} \in c(M \backslash D)$. Last we concatenate $\mathbf{v}_{0^{-}} \cdots \mathbf{v}_{j}$ and $\mathbf{d}_{l} \cdots \mathbf{d}_{m}$ and $\mathbf{v}_{k+1^{\prime}} \cdots \mathbf{v}_{n}$ to obtain a path $\mathbf{v}_{0^{-}} \cdots \mathbf{v}_{n} \subseteq$ $c(M \backslash D)$ such that $\mathbf{v}_{0}=\mathbf{v}$ and $\mathbf{v}_{n}=\mathbf{v}^{\prime}$.

Now we show Theorem 14 .

First we show that $\partial(A \cup B)$ is a connected 2-manifold. Thanks to Theorem 10, $\partial(A \cup B)$ is a 2-manifold. Furthermore $\partial A \backslash D$ is connected and $\partial B \backslash D$ is connected (Lemma 20) and $\emptyset \neq c(\partial D) \subseteq c(\partial A \backslash D) \cap c(\partial B \backslash D)$ (thanks to Lemma 19). Thus $\partial(A \cup B)=(\partial A \backslash D) \cup(\partial B \backslash D)$ is connected.

Second we show that $c(D) \cap c(\partial(A \cup B))=c(\partial D)$. Lemma 19 using $M \in\{\partial A, \partial B\}$ implies $c(D) \cap c(\partial A \backslash D)=c(\partial D)=$ $c(D) \cap c(\partial B \backslash D)$. Therefore

$$
c(D) \cap(c(\partial A \backslash D) \cup c(\partial B \backslash D))=c(\partial D) .
$$

We obtain the result using Lemma 15 .

Third we show that $\chi(c(\partial(A \cup B)))=\chi(c(\partial A))$. Thanks to Lemma 15, $D \cup \partial(A \cup B)=\partial A \cup \partial B$. Since $\chi(X \cup Y)=$ $\chi(X)+\chi(Y)-\chi(X \cap Y)$,

$$
\chi(c(D))+\chi(c(\partial(A \cup B)))-\chi(c(D) \cap c(\partial(A \cup B)))
$$

4 Addendum: we also assume that $M$ is a set of triangles.

5 Addendum: $\mathbf{v}$ is a redundant notation of $\sigma$.

6 Addendum: we also assume that $M$ is a set of triangles. 


\begin{tabular}{|c|cccc|cccc|cccc|}
\hline & 1 & 2 & 3 & 4 & 5 & 6 & 7 & 8 & 9 & 10 & 11 & 12 \\
\hline $\mathrm{x}$ & 1 & 0 & -1 & 0 & 2 & 0 & -2 & 0 & 1 & 0 & -1 & 0 \\
$\mathrm{y}$ & 0 & 1 & 0 & -1 & 0 & 2 & 0 & -2 & 0 & 1 & 0 & -1 \\
$\mathrm{z}$ & 2 & 1 & 2 & 1 & 3 & 0 & 3 & 0 & 0 & 3 & 0 & 3 \\
\hline
\end{tabular}

Table 13 D coordinates of the 12 vertices in $V$.

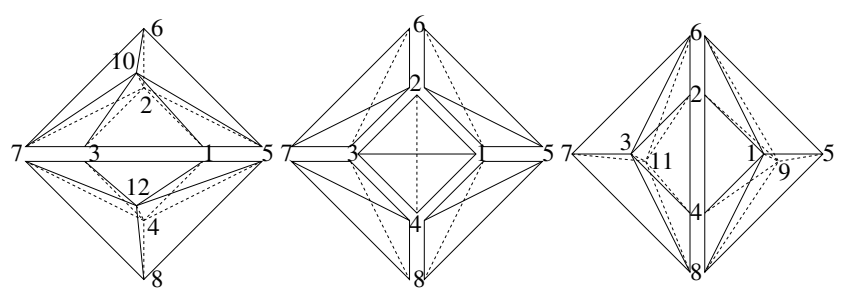

Fig. 19 Top views of the three subsets of $Z$. Left: the top subset is the union of the tetrahedra cycles $Z_{2.10}$ and $Z_{4.12}$. Middle: the center subset has five internal tetrahedra. Right: the bottom subset is the union of the tetrahedra cycles $Z_{1.9}$ and $Z_{3.11}$. Note that vertices are sometimes duplicated.

$$
=\chi(c(\partial A))+\chi(c(\partial B))-\chi(c(\partial A) \cap c(\partial B)) .
$$

Furthermore $^{7}, \chi$ is known for 2-balls, cycles and 2-spheres:

$$
\begin{aligned}
& \chi(c(D))=\chi(c(\partial A) \cap c(\partial B))=1, \\
& \chi(c(D) \cap c(\partial(A \cup B)))=\chi(c(\partial D))=0, \\
& \chi(c(\partial B))=2 .
\end{aligned}
$$

Thus

$1+\chi(c(\partial(A \cup B)))-0=\chi(c(\partial A))+2-1$.

Now we see that $\partial A$ and $\partial(A \cup B)$ have the same genus.

\section{G An example for Theorem 15}

First we remind how $Z$ is constructed in [24] using shortened notations: a vertex is an integer between 1 and 12 , the tetrahedron with vertices $\{1,2,3,4\}$ is written 1.2.3.4, the edge with vertices $\{1,2\}$ is written $1.2,1.2 \times 3-4-5-6$ is the tetrahedron set $\{1.2 .3 .4,1.2 .4 .5,1.2 .5 .6\}$ etc. Let $V$ be the set of the vertices $1,2 \cdots 12$. The $3 \mathrm{D}$ coordinates of the vertices in $V$ are given in Tab. 1. Then $Z$ is defined as the disjoint union of three subsets of tetrahedra (Fig. 19). The center subset is

$$
Z_{0}=\{1.2 .3 .4,1.2 .5 .6,2.3 .6 .7,3.4 .7 .8,4.1 .8 .5\} \text {. }
$$

The top subset is the union of the tetrahedron cycles $Z_{2.10}$ and $Z_{4.12}$ :

$Z_{2.10}=2.10 \times 5-6-7-3-1-5, Z_{4.12}=4.12 \times 7-8-5-1-3-7$.

The bottom subset is the union of the tetrahedron cycles $Z_{1.9}$ and $Z_{3.11}$ :

$Z_{1.9}=1.9 \times 5-6-2-4-8-5, Z_{3.11}=3.11 \times 7-8-4-2-6-7$.

We obtain

$Z=Z_{0} \cup Z_{1.9} \cup Z_{2.10} \cup Z_{3.11} \cup Z_{4.12}$.

Second we check that $Z$ is included in a set of tetrahedra $T$ such that $c(T)$ is a $3 \mathrm{D}$ Delaunay triangulation.

7 Addendum: since $c(A) \cap c(B)$ is a 2-ball (assumption of Theorem 14) and $c(A) \cap c(B)=c(\partial A) \cap c(\partial B)$ (thanks to Lemma $18, c(\partial A) \cap c(\partial B)$ is a 2 -ball.
Lemma 21 Thanks to Tab. 1, Z is included in a 3D Delaunay triangulation whose vertex set is $V$.

Proof Let $\mathbf{c}_{\Delta}$ and $r_{\Delta}$ be the center and radius of the circumscribing sphere of the tetrahedron $\Delta$. Let

$e=\min _{\Delta \in Z, \mathbf{u} \in V \backslash c(\Delta)} d^{2}\left(\mathbf{u}, \mathbf{c}_{\Delta}\right)-r_{\Delta}^{2}$,

where $d$ is the Euclidean distance between two 3D points. We have $e=0.5>0$. Thus, for every $\Delta \in Z$, there is no $V \backslash c(\Delta)$ vertex in the (interior of the) sphere that includes the four $\Delta$ vertices. Therefore $Z$ is included in a 3D Delaunay triangulation of $V$.

Third we show that $Z$ is the union ${ }^{8}$ of four pipes and a 3-ball.

Lemma 22 We have $Z=P \cup S$ where

$$
\begin{aligned}
& P= p(5.9 .1,6.2 .10) \cup p(7.11 .3,6.2 .10) \cup \\
& p(5.9 .1,8.4 .12) \cup p(7.11 .3,8.4 .12), \\
& S=\{1.2 .3 .4,1.9 .2 .4,2.10 .1 .3,3.11 .2 .4,4.12 .1 .3\} \\
& \text { and } S \text { is a } 3 \text {-ball }{ }^{9} .
\end{aligned}
$$

Proof Eq. 15 is rewritten as

$p\left(\mathbf{a}_{0} \mathbf{b}_{0} \mathbf{c}_{0}, \mathbf{a}_{1} \mathbf{b}_{1} \mathbf{c}_{1}\right)=\mathbf{b}_{0} \mathbf{c}_{0} \times \mathbf{a}_{0}-\mathbf{a}_{1}-\mathbf{b}_{1} \cup\left\{\mathbf{a}_{0} \mathbf{a}_{1} \mathbf{b}_{1} \mathbf{c}_{0}\right\} \cup$ $\mathbf{b}_{1} \mathbf{c}_{1} \times \mathbf{c}_{0}-\mathbf{a}_{0}-\mathbf{a}_{1}$.

Thanks to Eq. 53,

$p(5.9 .1,6.2 .10)=9.1 \times 5-6-2 \cup\{5.6 .2 .1\} \cup 2.10 \times 1-5-6$ $p(7.11 .3,6.2 .10)=11.3 \times 7-6-2 \cup\{7.6 .2 .3\} \cup 2.10 \times 3-7-6$ $p(5.9 .1,8.4 .12)=9.1 \times 5-8-4 \cup\{5.8 .4 .1\} \cup 4.12 \times 1-5-8$ $p(7.11 .3,8.4 .12)=11.3 \times 7-8-4 \cup\{7.8 .4 .3\} \cup 4.12 \times 3-7-8$.

Now we see that

$$
\begin{aligned}
& P=\left(Z_{0} \backslash\{1.2 .3 .4\}\right) \cup \\
& 9.1 \times 2-6-5-8-4 \cup 2.10 \times 1-5-6-7-3 \cup \\
& 11.3 \times 2-6-7-8-4 \cup 4.12 \times 1-5-8-7-3 \\
& =\left(Z_{0} \backslash\{1.2 .3 .4\}\right) \cup\left(Z_{1.9} \backslash\{1.9 .2 .4\}\right) \cup \\
& \left(Z_{2.10} \backslash\{2.10 .1 .3\}\right) \cup\left(Z_{3.11} \backslash\{3.11 .2 .4\}\right) \cup \\
& \left(Z_{4.12} \backslash\{4.12 .1 .3\}\right) \\
& =Z \backslash S \text {. }
\end{aligned}
$$

and $S \subset Z$. Note that $S$ is a 3 -ball since it has a shelling started by the central tetrahedron 1.2.3.4 and the four other tetrahedra $\Delta_{i}$ are added to $O_{i}$ such that $c\left(\Delta_{i}\right) \cap c\left(O_{i}\right)=c\left(t_{i}\right)$ where $t_{i} \in \partial 1.2 .3 .4$.

Fourth, we show that $c(S) \cap c(P)$ is an annulus.

Lemma 23 We have $c(S) \cap c(P)=c(N)$ where

$N=\{\mathbf{9 . 2 . 1}, 2.1 .10\} \cup\{3 . \mathbf{2 . 1 1}, \mathbf{1 0 . 3} .2\} \cup$

$\{11.4 .3,4.3 .12\} \cup\{1.4 .9,12.1 .4\}$.

The vertices in bold fonts form edges in $\partial N$ :

$\partial N=9-2-11-4-9 \cup 1-10-3-12-1$.

Proof Let $\sigma \in c(S) \cap c(P)$ be a simplex. Since vertices $5,6,7,8$ are not in $c(S), \sigma$ does not have a vertex in $\{5,6,7,8\}$. According $^{10}$ to Eq. 16 , the only triangles in $\partial p\left(\mathbf{a}_{0} \mathbf{b}_{0} \mathbf{c}_{0}, \mathbf{a}_{1} \mathbf{b}_{1} \mathbf{c}_{1}\right)$ that does not have vertices $\mathbf{a}_{0}$ and $\mathbf{a}_{1}$ are $\left\{\mathbf{b}_{0} \mathbf{b}_{1} \mathbf{c}_{0}, \mathbf{b}_{1} \mathbf{c}_{0} \mathbf{c}_{1}\right\}$.

\footnotetext{
8 Addendum: $Z$ is the disjoint union.

9 Addendum: we also have $S \cap P=\emptyset$ (thanks to Eq. 54).

10 Addendum: thanks to Eq. 14, if $\sigma \in c\left(p\left(\mathbf{a}_{0} \mathbf{b}_{0} \mathbf{c}_{0}, \mathbf{a}_{1} \mathbf{b}_{1} \mathbf{c}_{1}\right)\right)$ has neither vertex $\mathbf{a}_{0}$ nor $\mathbf{a}_{1}, \sigma \in c\left(\mathbf{b}_{0} \mathbf{b}_{1} \mathbf{c}_{0}, \mathbf{b}_{1} \mathbf{c}_{0} \mathbf{c}_{1}\right)$.
} 
Using Eq. 51 and $\mathbf{a}_{0} \in\{5,7\}$ and $\mathbf{a}_{1} \in\{6,8\}, \sigma$ is included is $^{11}$ one of the triangles in $N$ where

$\tilde{N}=\{9.2 .1,2.1 .10\} \cup\{11.2 .3,2.3 .10\} \cup$

$\{9.4 .1,4.1 .12\} \cup\{11.4 .3,4.3 .12\}$.

Therefore $c(S) \cap c(P) \subseteq c(\tilde{N})$. Conversely ${ }^{12}, \tilde{N} \subseteq c(P)$ and $\tilde{N} \subseteq c(S)$ implies $c(\tilde{N}) \subseteq c(S) \cap c(P)$. Last we check that $N=\tilde{N}$.

Thus $Z=P \cup S$ with $n=4$ and $P=\cup_{i} P_{i}{ }^{13}$ as in Theorem 15 . Since $Z$ is strongly non-shellable [24], $Z=P \cup S^{\prime}$ and $S=S^{\prime}$.

\section{H Addendum}

- Title "2-Manifold criterion for sets of triangles" is better than "2-Manifold criterion for simplicial complexes" for Sec. 2.5, since $X$ in Sec. 2.5 is a set of triangles ( $X$ is not a simplicial complex).

- We need a basic Lemma two times in the paper:

Lemma 24 If a simplicial complex $X \subset c(T)$ is a 2manifold with boundary, $X$ is $2 D$ pure.

Intuitively, if $X$ is not $2 \mathrm{D}$ pure, there is a point in $|X|$ that has no 2D neighborhood in $|X|$. A proof is in Sec. H.1.

- Lemma 24 is used at the very end of Appendix D using $X=c(\partial A) \cap c(\partial B)$.

- Lemma 24 is used at the very beginning of Appendix $\mathrm{F}$ using $X=c(A) \cap c(B)$. Since this $X$ is a 2-ball, $X$ meets the Lemma's assumptions and $|X|$ is the union of the triangles in $X$. Since the set of these triangles is $D=$ $\partial A \cap \partial B$, we see that $D$ is a 2 -ball.

\section{H.1 Proof of Lemma 24}

Assume (reductio ad absurdum) that $X$ is not $2 \mathrm{D}$ pure. Thus there is a vertex that is not in an edge (both in $X$ ), or there is an edge that is not in a triangle (both in $X$ ). Since $X$ is a 2 -manifold with boundary, the vertex point is homeomorphic to $\mathbb{R}^{2}$ or $\mathbb{R} \times \mathbb{R}^{+}$in the first case (impossible). In the second case, we consider the middle point $\mathbf{m}$ of the edge and one of its small neighborhood $N$ in $|X|$ that is included in the edge such that $N$ is homeomorphic to $\mathbb{R}^{2}$ or $\mathbb{R} \times \mathbb{R}^{+}$. Now we see that $N \backslash\{\mathbf{m}\}$ is homeomorphic to $\mathbb{R}^{2}$ minus a point or homeomorphic to $\mathbb{R} \times \mathbb{R}^{+}$minus a point. This is impossible since the former is disconnected and the latters are connected.

\section{References}

1. Boissonnat J.D.: Geometric structures for threedimensional shape representation. ACM Transactions on Graphics 3(4), 266-286 (1984)

2. Boissonnat J.D., Devillers O., Pion S., Teillaud M., Yvinec M.: Triangulations in CGAL. Computational Geometry: Theory and Applications 22(5) (2002)

3. Bruggesser. H., Mani P.: Shellable decomposition of cells and spheres. Math. Scand. 29, 197-205 (1971)

11 Addendum: replace "is" by "in".

12 Addendum: the four equations just below Eq. 53 imply $\tilde{N} \subseteq c(P)$, the $S$ definition in Lemma 22 implies $\tilde{N} \subseteq c(S)$.

13 Addendum: $P_{0}, P_{1}, P_{2}$ and $P_{3}$ are those in Eq. 51 .
4. Danaraj G., Klee V.: A representation of 2-dimensional pseudomanifolds and its use in the design of a linear-time shelling algorithm. Algorithmic aspects of combinatorics, vol.2 of ann. Discrete Math., 53-63 (1978)

5. DeRose T., Duchamp T., Hoppe H., McDonald J.A., Stuetzle W: Reconstructing two-dimensional manifolds from scattered data: motivations and background. Technical report 215, University of Washington (1991)

6. Diestel R.: Graph theory. Springer-Verlag, Graduated Texts in Mathematics, Heidelberg (2010)

7. Edelsbrunner H.: An acyclicity theorem for cell complexes in d dimensions. Combinatorica 10(3), 251-260 (1990)

8. Edelsbrunner H.: Geometry and topology for mesh generation. Cambridge University Press (2001)

9. Gallier J.: Notes on convex sets, polytopes, polyhedra, combinatorial topology, voronoi diagrams and Delaunay triangulation. Research report RR-6379, INRIA (2007)

10. Gezahegne A.: Surface reconstruction with constrained sculpting. Master of science thesis, University of California (2005)

11. Giblin P.: Graphs, surfaces and homology. Cambridge University Press, third edition (2010)

12. Hachimori M.: Combinatorics of constructible complexes. $\mathrm{PhD}$. thesis, University of Tokyo (2000)

13. Lhuillier M., Yu S.: Manifold surface reconstruction of an environment from sparse structure-from-motion data. CVIU 117(11), 1628-1644 (2013)

14. Lhuillier M.: 2-manifold tests for 3D Delaunay triangulation-based surface reconstruction. Journal of Mathematical Imaging and Vision 51(1) (2015)

15. Litvinov V., Lhuillier M.: Incremental solid modeling from sparse and omnidirectional structure-from-motion data. BMVC (2013).

16. Litvinov V., Lhuillier M.: Incremental solid modeling from sparse structure-from-motion data with improved visual artifacts removal. ICPR (2014).

17. Lutz F.H.: Combinatorial 3-manifold with 10 vertices. Beitrage zur Algebra und Geometrie 49(1), 97-106 (2008)

18. Romanoni A., Matteucci M.: Incremental reconstruction of urban environments by edge-point Delaunay triangulation. IROS (2015).

19. Sakai K.: Simplicial homology, a short course. Institute of Mathematics, University of Tsukuba (2010).

20. Varadhan G., Krishnan S., Sriram TVN., Manocha D.: Topology preserving surface extraction using adaptive subdivision. Eurographics Symposium on Geometry Processing (2004).

21. Vegter G.: Computational topology. In GoodMan J. Rourke J.O. (eds) Handbook of discrete and computational geometry, CRS. Press (2004)

22. Xia J., He. Y., Han S., Fu. C.W., Luo. F., Gu. X.: Parameterization of a star-shaped volumes using green's functions. Lecture Notes in Computer Science 6130, 219-235, (2010)

23. Yu S., Lhuillier M.: Incremental reconstruction of a manifold surface from sparse visual mapping. 3DIMPVT (2012).

24. Ziegler G.M: Shelling polyhedral 3-balls and 4-polytopes. Discrete Computational Geometry 19, 159-174 (1998)

25. Ziegler G.M.: Lectures on polytope. Springer, Graduate Texts in Mathematics, Berlin (2007) 